\title{
FATIGUE IN CHRONIC HEPATITIS C INFECTION \\ A MIXED METHOD STUDY
}

\author{
by
}

Dora Marta Zalai

MD, Semmelweis University, 1998; Dipl Psych, The Open University, 2005

\author{
A thesis \\ presented to Ryerson University \\ in partial fulfillment of the \\ requirements for the degree of \\ Master of Arts \\ in the Program of \\ Psychology (Clinical Stream)
}

Toronto, Ontario, Canada (2014)

(c) (Dora Zalai) 2014 


\section{Author's Declaration}

I hereby declare that I am the sole author of this thesis.

I authorize Ryerson University to lend this thesis to other institutions or individuals for the purpose of scholarly research.

I further authorize Ryerson University to reproduce this thesis by photocopying or by other means, in total or in part, at the request of other institutions or individuals for the purpose of scholarly research. 


\begin{abstract}
Thesis title: Fatigue in Chronic Hepatitis C Infection - A Mixed Method Study

Degree and Year of Convocation: Master of Arts, 2014
\end{abstract}

Full Name: Dora Marta Zalai

Graduate Program and University: Psychology (Clinical Stream), Ryerson University

Fatigue is a main patient reported outcome of chronic hepatitis C (HCV) infection; yet its

contributors are unknown. Objectives: The study (1) evaluated fatigue predictors, (2) tested the mediating role of fatigue cognitions, (3) screened for sleep disorders, and (4) explored fatigue from patients’ perspectives. Participants: Both sexes (age >18 years, $N=115$ ) with chronic HCV infection. Design: Cross-sectional. Results: Sixty percent reported severe fatigue (FSS $\geq 4$ ). Fatigue perceptions were the main predictors of fatigue $(\Omega=.58$, bias corrected $C I=.070-.163)$. Fatigue perceptions mediated the relationship between comorbidities and fatigue. Half of the sample reported clinically significant symptoms of insomnia and/or sleep apnea. Eight main fatigue themes were endorsed by the participants. Conclusions: Fatigue and sleep disorders were clinically significant issues. Fatigue cognitions may contribute to severe fatigue outcomes. Significance: Integrating the findings into existing sleep and fatigue treatments could improve clinical outcomes. 


\section{Acknowledgements}

I am indebted to Dr. Colleen Carney, for providing me the opportunity to study clinical psychology. She has given me the greatest gift by making the dream of my life come true. I am grateful for her trust, patience, and for teaching me by example the value of scientific rigour and professional excellence. I would like to express my gratitude to Dr. Kelly McShane for her wisdom, kindness, and encouragement. She gently guided me through this project ensuring that I kept both my focus and passion. I am truly thankful to Dr. Morris Sherman for giving me the opportunity to conduct this study in his clinic and for his substantial support. I thank Dr. Tae Hart for her interest in the topic, for her kind support and insightful comments. I was helped by Katalin Fornadi with data collection and entry and I am appreciative of this. I am indebted to Dr. Colin Shapiro for his support. He sparked my interest in the field of sleep and taught me to consider the big picture and to never give up. I appreciate the time and knowledge given by the patients who participated in the study. 
To patients with chronic hepatitis $C$ infection and to those who treat them. 
$\begin{array}{ll}\text { Introduction } & 1\end{array}$

$\begin{array}{ll}\text { Overview } & 1\end{array}$

$\begin{array}{ll}\text { Defining Fatigue } & 2\end{array}$

Definitions of Fatigue in the Scientific Literature $\quad 2$

The Assessment of Fatigue $\quad 5$

The Measurement of Physiological Fatigue $\quad 5$

The Assessment of Psychological Fatigue $\quad 6$

$\begin{array}{ll}\text { Fatigue in Chronic General Medical Conditions } & 10\end{array}$

Fatigue in Chronic Hepatitis C Infection $\quad 11$

$\begin{array}{ll}\text { Study Rationale } & 18\end{array}$

$\begin{array}{ll}\text { Theoretical Lens } & 19\end{array}$

$\begin{array}{ll}\text { Thesis Hypotheses and Objectives } & 21\end{array}$

$\begin{array}{ll}\text { Methods } & 22\end{array}$

$\begin{array}{ll}\text { Mixed Methods Approach } & 23\end{array}$

$\begin{array}{ll}\text { Participant Characteristics } & 24\end{array}$

$\begin{array}{ll}\text { Procedures } & 26\end{array}$

$\begin{array}{ll}\text { Sample Size and Power } & 27\end{array}$

$\begin{array}{ll}\text { Measures, Interviews and Materials } & 27\end{array}$

$\begin{array}{ll}\text { Questionnaires } & 27\end{array}$

$\begin{array}{ll}\text { Interviews } & 37\end{array}$ 
Quantitative Analysis

Qualitative Analysis

Mixed Methods Analysis

Results

Quantitative Results

Qualitative Results

Mixed Method Results

Discussion

69

Tables

Figures 107

Appendixes

118

References

Abbreviations

170 


\section{List of Tables}

Table 1 The Subtypes and Assessment of Physiological and Psychological Fatigue 89

$\begin{array}{lll}\text { Table } 2 & \text { Normal and Pathological Fatigue } & 90\end{array}$

Table 3 Factors Contributing to Fatigue in General Medical Conditions 91

Table $4 \quad$ Participant Characteristics 92

Table 5 Participant Characteristics - Qualitative sample 93

Table $6 \quad$ Descriptive Statistics of Study Variables 94

Table $7 \quad$ Distribution of ISI Scores 95

Table 8 Distribution of DASS Scores 96

Table $9 \quad$ Factor Loadings for Principal Component Analysis with Varimax 97 Rotation of the Brief Illness Perception Questionnaire

$\begin{array}{lll}\text { Table } 10 & \text { Correlation Amongst Study Variables } & 98\end{array}$

Table 11 Hierarchical Multiple Regression Models Predicting Fatigue 99

Table 12 Final Hierarchical Multiple Regression Model Predicting Fatigue 101

Table 13 Results of the Exploratory Mediation Analyses 102

Table 14 Differences in Fatigue and Sleepiness in Insomnia and Sleep Apnea 103

Table 15 Qualitative Themes 104

Table 16 The Most Important Fatigue Attributions 106 


\section{List of Figures}

Figure 1 Conceptual Framework for Understanding Fatigue 107

$\begin{array}{lll}\text { Figure } 2 & \text { The Two Stage Model of Fatigue in Medical Conditions } & 108\end{array}$

$\begin{array}{lll}\text { Figure } 3 & \text { The Scatterplot of Fatigue and Sleepiness Scores } & 109\end{array}$

Figure 4 The Results of the Survey Question on the Possible Benefits of a Fatigue 110 Management Service at the Liver Clinic

Figure 5 The Distribution of STOP-BANG Scores 111

Figure 6 General Mediation Model with Multiple Mediators 112

$\begin{array}{lll}\text { Figure } 7 & \text { Mediation model } 1 & 113\end{array}$

$\begin{array}{lll}\text { Figure } 8 & \text { Mediation model } 2 & 114\end{array}$

$\begin{array}{lll}\text { Figure } 9 & \text { Mediation model } 3 & 115\end{array}$

Figure 10 Insomnia and Obstructive Sleep Apnea Screening Results 116

$\begin{array}{lll}\text { Figure } 11 & \text { Fatigue Perception Model } & 117\end{array}$ 


\section{List of Appendices}

Appendix A Commonly Used Fatigue Questionnaires

Appendix B Interview Guide

124

Appendix C Code Books

Appendix D An Example for a Fatigue Screening Sheet

Appendix E Fatigue Perception Model 


\section{Fatigue in Chronic Hepatitis C Infection: A Mixed Design Study}

\section{Overview}

In order to understand the problem of fatigue in chronic hepatitis $\mathrm{C}$ virus (HCV) infection, one has to clarify the nature of fatigue and how it presents in the medical context. To this end, the concept of fatigue is examined in sections one and two; in the first section as a lay construct then, in the second section as a scientific phenomenon. These sections will show that fatigue has multiple meanings in everyday language and these meanings vary across cultures and historically. In the Western scientific literature, fatigue is defined in terms of discrete categories of physical versus psychological or normal versus pathological fatigue. These scientific conceptualizations influence the ways in which fatigue is measured. This is described in section three. The focus in section four shifts to fatigue, as a patient-reported symptom, in general medical conditions. The synthesis of this section is that fatigue is a significant issue for patients and usually arises as a common shared pathway among multiple psychological factors and comorbid medical conditions. As will be elaborated in the final section, fatigue reported by patients with chronic hepatitis $\mathrm{C}$ virus (HCV) infection shares some of these general features. However, only a limited number of the possible contributing factors have been studied and the phenomenology of fatigue has been relatively neglected (given the impact and salience of fatigue in general in and this population in particular). The latter point provides the rationale for the study presented in this thesis: that is, in order to understand the nature of fatigue in HCV infection, the phenomenology and specific contributing factors of fatigue need to be systematically explored. The argument for embarking on this endeavour is that fatigue is among the leading complaints in the HCV infected population and if not knowledgably assessed and managed, it may have a negative impact on patient-reported disease outcomes. 


\section{Defining Fatigue}

\section{Definitions of Fatigue in the Scientific Literature}

The categorization of fatigue as either a physiological or a psychological phenomenon is currently the prevailing approach in science (Table 1).

Insert Table 1 here

The concepts of physiological and psychological fatigue are then further segmented by the disciplines of science that provide distinct perspectives and conceptual models for understanding fatigue (Figure 1). The physiological and psychological conceptualizations of fatigue are elaborated below.

\section{Insert Figure 1 here}

Physiological fatigue. Physiological fatigue in the basic sciences has been defined as a temporal loss of voluntary force-generating capacity during exercise (Vollestad, 1997). The declining force in the muscles during contractions can originate from the impairment of the muscles or the neuro-muscular junction (peripheral fatigue) or from decreased central input (central fatigue or central activation failure). The conceptualization of fatigue in neurology is similar to the above model. As in physiology, peripheral fatigue is attributed to impaired neuromuscular transmission, neuro-metabolic disorders or disorders in the contractile mechanisms but a broader, neurological conceptualization also includes diseases of the peripheral, afferent nerves and of the lower motor neuron (Chaudhuri \& Behan, 2004). From a neurological perspective, 
central fatigue is defined as muscle fatigability due to decreased input from the nervous system at or above the level of the upper motor neuron.

A yet broader conceptualization framework describes peripheral (physical) fatigue as a decreased ability to maintain force because of impairment of the muscles, cardiovascular or the nervous system without impairment of sustained mental tasks. Central fatigue, on the other hand is defined as a difficulty with initiating or maintaining cognitive or physical activities that require self-motivation (Chaudhuri \& Behan, 2000).

Psychological fatigue. Psychological (experienced, subjective, or central) fatigue is the lived experience of fatigue that is constructed by our perceptions of the internal and external environment, our mood, life experiences, our knowledge and belief systems. The definitions of psychological fatigue typically refer to a multi-dimensional construct, a subjective sense of energy or effort imbalance, and functional impairment as, for example "an overwhelming sense of tiredness, lack of energy and a feeling of exhaustion, associated with impaired physical and or cognitive functioning” (Shen, Barbera, \& Shapiro, 2006, p.70)

The above definitions of physiological and psychological fatigue imply that fatigue is a pathological condition associated with "overwhelming” sensations and anatomical or functional impairment. Alternatively, fatigue can we viewed as both a normative experience and a pathological condition.

Normal and pathological fatigue. A simple distinction between normal and pathological fatigue is based on characteristics of duration, associated conditions, functional impairment, and alleviation by usual adaptive behaviours (Shen, Barbera, \& Shapiro, 2006) (Table 2).

Insert Table 2 here 
The shortcoming of this approach is that some fatigue conditions do not fit neatly to these categories. For example, fatigue associated with acute viral infections could be considered normal, since it is temporary and is alleviated by rest. However, also it could be categorized as pathological in that it is associated with an illness and is functionally impairing.

From a neurophysiological perspective, fatigue has been depicted as an essentially normal adaptation mechanism (Chaudhuri \& Behan, 2004). According to this model, perceived exertion has an optimal set point that is maintained by the balance between the amount of voluntary effort and work output. This balance is the function of the interaction among control factors in the internal and external environment (e.g., hormone levels, humidity, temperature) and the effortless information flow among sensory systems, the efferent motor system and brain areas regulating motivation and voluntary motor control. Pathological fatigue is an enhanced sense of normal perceived exhaustion produced by a homeostatic imbalance due to abnormal changes in the system at any level. For example, decreased motivational input leads to perceived fatigue in depression; diseases of the motor cortex, neurons, or muscles results in fatigue in neurological disorders; changes in hormone levels cause fatigue in endocrine diseases or working in a hot, humid environment is associated with amplified level of perceived exhaustion.

Finally, as it has been alluded to above, whether fatigue is regarded as a normal experience or a pathological condition is influenced by socio-cultural traditions and beliefs. These beliefs and attributions influence whether individuals seek medical help when feeling fatigued and whether they receive medical care for their fatigue (and if so, which specialist they are sent to and what will be the focus of treatment). 


\section{The Assessment of Fatigue}

The conceptualization of fatigue guides the choices of measurement methods used to assess it (Table 1).

\section{The Measurement of Physiological Fatigue}

Physiological fatigue is measured by electrophysiological methods. A force transducer, for example measures peripheral fatigue as reduction of actual force produced by the muscle in response to electrical stimulation following exercise. An alternative, non-invasive method is using surface electromyography (sEMG) which allows amplitude, frequency and muscle-fibre conduction velocity to be measured online, simultaneously in several muscles. Central physiological fatigue can be measured as a decreased output of the motor cortex after magnetic stimulation after fatiguing mental activity (Gandevia, 2001). A more simple measure of central fatigue is readiness potential: a movement-related negative cortical electroencephalograph (EEG) potential appearing approximately one second before a voluntary motor act. The amplitude of the readiness potential is related to the voluntary input and perceived effort and has been shown to change when individuals are engaged in fatigue-inducing physical tasks (Schillings et al., 2007).

The objective measurement of cognitive (central or mental) fatigue, as defined by decline of mental performance over the course of mentally demanding cognitive tasks, has traditionally been measured in neuropsychological experiments. In these situations, fatigue is typically induced in following ways: (a) prolonged mental effort, e.g., participation in prolonged neuropsychological testing (typically 3-5 hours in length); (b) sustained mental effort, e.g., sustaining maximal, or other determined level of mental performance during neuropsychological testing; (c) performing challenging mental tasks; or (d) performing challenging physical tasks. 
Alternatively, cognitive performance is measured after prolonged wakefulness, for example after partial or total sleep deprivation.

An intriguing pattern emerging from these experiments is that fatigue induction leads to cognitive fatigue but there is no consistent difference in the cognitive performance between groups with medical conditions in which fatigue is a characteristic feature and healthy controls (Leavitt \& DeLuca, 2010). In other words, patients reporting moderate-severe fatigue before or over the course of testing, show similar performance to those healthy individuals who do not report increased fatigue. These findings raise the possibility that physiological and psychological fatigue are separate phenomena or that experiential fatigue is related to, but is a more complex phenomenon than, physiological fatigue.

\section{The Assessment of Psychological (Experienced, Subjective, Central) Fatigue}

Questionnaires. The most widespread approach for the assessment of experienced fatigue is using self-report scales and questionnaires. Since there may be different types of fatigue and there is not a single, "gold standard" definition of experienced fatigue, the fatigue questionnaires assess distinct fatigue constructs. A summary of the most frequently used questionnaires is found in Appendix A. The questionnaires are printed in the STOP, THAT and One Hundred Other Sleep Scales (Shahid, Wilkinson, Marcu, \& Shapiro, 2011).

Some tools (for example the Fatigue Assessment Scale) are based on the assumption that fatigue is a unidimensional construct; others are designed for the assessment fatigue as a multifaceted phenomenon (Fisk et al., 1994; Michielsen, De Vries, \& Van Heck, 2003; Schwartz, Jandorf, \& Krupp, 1993; Smets, Garssen, Bonke, \& De Haes, 1995). The multidimensional fatigue scales distinguish between for example physical, cognitive and motivational fatigue as well as assess the impact of fatigue on everyday functioning. There is a 
variation in the items belonging to the abovementioned dimensions of fatigue, sometimes similar items falling in different categories. The Chalder Fatigue Scale, for example, has "physical symptoms" and "mental symptoms" subscales and it lists both the general feeling of tiredness and motivational fatigue under "physical symptoms”(Chalder et al., 1993). In contrast, the Multidimensional Fatigue Inventory separates the core feeling of tiredness into a "general fatigue" category and links the motivational fatigue to the "reduced motivation” subscale (Smets, et al., 1995).

An important issue related to construct validity is that some questionnaires do not differentiate between sleepiness and fatigue despite the fundamental differences between the two constructs. Sleepiness correlates with a homeostatic drive to sleep and traditionally is measured as the degree of sleep propensity under standard circumstances in the sleep laboratory. The differentiation between sleepiness and fatigue is crucial in clinical assessment, since they have different pathophysiology, causes, consequences, and clinical implications. For example, certain sleep disorders (e.g. narcolepsy) are predominantly associated with excessive daytime sleepiness whereas others (e.g. insomnia) are more related to daytime fatigue. When both are present, the alleviation of excessive daytime sleepiness should be the treatment priority, since it increases the risk for motor-vehicle and occupational accidents (Teran-Santos, Jimenez-Gomez, \& CorderoGuevara, 1999). Differentiation between sleepiness and fatigue is also a key element of the cognitive-behavioural therapy for insomnia. Fatigue questionnaires that include items about sleepiness, for example the Chalder Fatigue Scale and the Fatigue Impact Scale, or other scales that were validated using established sleepiness scales, for instance the Visual Analogue Scale for Fatigue, neglect this important distinction (Chalder, et al., 1993; Lee, Hicks, \& Nino-Murcia, 1991; Schwartz, et al., 1993). One can argue that even the questionnaires using the word 
"tiredness", which in lay language means both sleepiness and fatigue, may fail to differentiate between the two phenomena.

An abundance of fatigue scales are available for use in clinical populations. For example, The Brief Fatigue Inventory, the FACT-F subscale, the Rhoten Fatigue Scale, the Fatigue Symptom Inventory, the Swartz Cancer Fatigue Scale, and the Piper Fatigue Scale have specifically been developed to assess cancer-related fatigue (Hann et al., 1998; Mendoza et al., 1999; Piper et al., 1989; Rhoten, 1982; Schwartz, et al., 1993; Yellen, Cella, Webster, Blendowski, \& Kaplan, 1997). The Fatigue Severity Scale, the Fatigue Assessment Instrument, the Fatigue Impact Scale and the Fatigue Questionnaire have been designed and validated for use in multiple medical conditions (Chalder, et al., 1993; Fisk, et al., 1994; Krupp, LaRocca, MuirNash, \& Steinberg, 1989a; Schwartz, et al., 1993).

Questionnaires provide a cost- and time effective way to assess fatigue in clinical practice and research. The choice of questionnaire should be based on the psychometric properties of the particular tests and on understanding of the characteristics of fatigue constructs they assess. It is important to note that responses are influenced by the respondents' interpretation of the items, their mood and recall bias. Unfortunately, most fatigue questionnaires omit to offer a definition of fatigue before prompting respondents to mark their answers. In order to circumvent recall bias, questionnaires or electronic devices (e.g. actigraph or cell phone) can be used to detect the momentary experience of fatigue several times a day (Buysse et al., 2007; Dimsdale, AncoliIsrael, Ayalon, Elsmore, \& Gruen, 2007). This Ecological Momentary Assessment Method can reveal individual fatigue profiles that retrospective methods may conceal.

Qualitative methods. Qualitative methods are ideal for understanding the subjective experience and functional impact of fatigue. The advantage of these methods is that individuals 
are free to use their own words to describe what fatigue means to them without being constrained by a limited choice of the questionnaire items. Inquiry about the individual's fatigue validates this experience and can also reveal the processes that link experiential fatigue to changes in function and quality of life. As will be described below, this is especially important in medical settings, where fatigue is among the most common patient-reported factors contributing to distress and disability. Indeed, patient focus groups have expressed a preference of describing their fatigue experience, including the tandem life changes and coping strategies with the health care team, rather than merely assigning numbers to fatigue symptoms on scales that do not fully capture the experiential aspects of their fatigue (Yorkston, Johnson, Boesflug, Skala, \& Amtmann, 2010). The value of qualitative research on fatigue has been demonstrated in several medical conditions. Qualitative studies have shown, for example that fatigue is the most frequently reported concern of individuals with chronic hepatitis C infection (Kleinman et al., 2012). Sleep restriction therapy may seem to be a low risk intervention for insomnia, but a qualitative study showed that patients may experience severe fatigue in the first week of the treatment (Kyle, Morgan, Spiegelhalder, \& Espie, 2011). Patients described their fatigue management behaviours and beliefs about fatigue in qualitative studies on chronic fatigue syndrome, rheumatoid arthritis and cancer (Anderson, Jason, Hlavaty, Porter, \& Cudia, 2012; Larun \& Malterud, 2007; Repping-Wuts, Uitterhoeve, van Riel, \& van Achterberg, 2008; Siegel, Lekas, \& Maheshwari, 2012; Spichiger, Rieder, Muller-Frohlich, \& Kesselring, 2012). These studies provided invaluable information for cognitive behavioural interventions. The assumption underlying these qualitative assessment methods is that individuals are reliable reporters of their internal states. One can argue though that these approaches may be biased, since individuals have 
limited insight to their internal reality or may be so much influenced by it that their self-report will be distorted.

\section{Fatigue in Chronic General Medical Conditions}

Fatigue is among the leading, most distressing, and debilitating symptom of many medical conditions (DeLuca, 2005). When fatigue is present in conjunction with a parallel disorder, it is usually considered to be the epiphenomenon of the primary medical condition. However, the relationship between the illness and fatigue appears to be more complex, in that it does not dissipate with recovery from the illness or it appears to be unrelated to the severity of the disorder.

The complexity of fatigue in the medically ill prompted the development of two-stage fatigue models. These models posit that the medical illness brings about biological changes that give rise to primary fatigue, which eventually becomes perpetuated by additional conditions, for instance depression, sleep disorders, and pain (De Luca, 2005; Kessel, \& Moss-Morris, 2006). (Figure 2).

Insert Figure 2 here

The contribution of depressive symptoms, anxiety, self-reported sleep problems and pain to fatigue has been shown in some general medical conditions (Table 3).

Insert Table 3 here 
The recent realization that the experience of fatigue involves high level mental processes represents a paradigm shift and opens a path to a different conceptualization for understanding fatigue. From a psychological perspective, patients' beliefs about their symptoms (beliefs about their illness, fatigue and their consequences) and views about themselves shape the coping behaviours they adopt in response to the illness and fatigue. These coping behaviours, in some cases, may be non-adaptive whereby they perpetuate rather than alleviate fatigue.

Cognitions and behavioural factors may also provide the causal link between secondary fatigue and conditions (e.g. insomnia, depression and anxiety) that perpetuate such fatigue. The cognitive-behavioural pathways by which secondary fatigue may be causally linked to its perpetuating conditions has not been systematically explored in the context of general medical conditions. The integration of these possible mechanisms to the two-stage fatigue model could provide a causal explanation of secondary fatigue and also could open new possibilities for a (cognitive behavioural) treatment of fatigue in the medically ill.

While the features and perpetuators of fatigue have received multi-disciplinary attention in some medical conditions, for example breast cancer and multiple sclerosis, it has been much less explored in other illnesses. The phenomenology and the perpetuating emotional, cognitive, and behavioural processes that provide the link between the primary physiological fatigue and the lived experience of chronic, secondary fatigue are especially neglected. Understanding the features and contributors of fatigue in these conditions are essential for the development of effective fatigue management strategies. Such a condition is chronic hepatitis C infection.

\section{Fatigue in Chronic Hepatitis C Infection}

The hepatitis C virus (HCV) has been endemic in humans for centuries but it was only discovered in 1989 (Choo et al., 1989; Kuo et al., 1989). Today, 130-170 million people live 
with chronic HCV infection worldwide. HCV-related liver failure causes 350,000 deaths annually (WHO, 2011). HCV causes a public health burden around the world. It spreads faster via shared needles, blood transfusion and reused medical supplies and has caused an epidemic five times larger than HIV (Gravitz, 2011). However, as opposed to HIV which has attracted significant public health attention and research funding, the awareness of the severity of the HCV epidemic is generally low (Gravitz, 2011). In countries, including Canada, where the screening of transfusion blood and use of disposable medical instruments resulted in a dramatic decline in the rate of new infections, the screening of those with high risk for infection (immigrants, injection drug users) is not widespread (Pottie et al., 2011). The rate of new infection in North America is highest in teenagers and young adults who may carry the virus for decades.

As of December 2007, approximately 242,500 Canadians had been infected with HCV (Public Health Agency of Canada, 2011). Forty percent of infected individuals live in Ontario. There has been an increase of approximately 8000 new cases annually between 2007 and 2010 countrywide; with the largest increase in annual rate of 107 new reported cases per 100,000 in the Yukon Territory. Transfusion with infected blood was the major cause of HCV infection in Canada until the introduction of second-generation anti- HCV assays in 1992. According to the latest Health Canada Statistics (2011), injection drug use (IDU) is associated with about twothirds of new infections, a further $14 \%$ of newly infected individuals contracts the disease through sexual contact with a HCV infected partner, nine percent get infected by drug snorting, and eight percent of new infections derive from other causes (including occupational exposure to blood, household contact with HCV carriers, incarceration without other risk factors). Ethnic minorities are disproportionately affected: immigration from countries where HCV infection is 
endemic contributes to 20-30\% of new cases in Canada and the reported rate of acute HCV infection is about five times as high in the Aboriginal versus the non-Aboriginal population.

There is no vaccine available for the prevention of HCV infection. The majority of infections stay undetected and become chronic since the virus replicates slowly and causes only a few, unspecific symptoms for years or decades. The chronic infection may gradually cause cirrhosis: the death and replacement of the liver cells by fibrotic tissue resulting in the decline of liver function, accumulation of toxins in the blood, jaundice, bleeding, accumulation of fluid in the abdomen, cognitive problems, and kidney failure. One to five percent of chronically infected patients will have liver failure or hepato-cellular carcinoma (liver cancer) (Wiegand \& Berg, 2013).

The standard treatment of HCV infection is the combination of weekly injection PEG interferon and daily oral ribavirin. The effectiveness of antiviral treatment depends on multiple factors, including the virus genotype and stage of liver disease. Despite advancements in the treatment method, approximately $55 \%$ of patients with genotype 1 infection and $20-25 \%$ of those with genotype 2 and 3 infections do not show sustained response to treatment (Sherman et al., 2007). Those who cannot be cured may develop specific symptoms and complications of chronic liver disease. In these cases, patient reported disease outcome measures, including quality of life, subjective well-being and functional capacity become the key issues of concern.

Chronic HCV infection is considered to be a largely asymptomatic disorder. On the other hand, research studies suggest that fatigue is among the most frequent patients-reported concerns in chronic HCV infection (Hilsabeck \& Malek-Ahmadi, 2004; Kleinman, et al., 2012). Whether the rate of fatigue is higher in the population with chronic hepatitis $\mathrm{C}$ infection than in the general population or the high rate reported in research reflects selection bias and weak study 
designs have been disputed (Wessely \& Pariante, 2002). Nevertheless, when persistent fatigue is present, it impacts on patients' quality of life and functional capacity (Foster, Goldin, \& Thomas, 1998; Kallman et al., 2007). Converging evidence indicates that there appears to be no direct relationship between the virus load and fatigue of patients with chronic HCV infection (Goh, Coughlan, Quinn, O'Keane, \& Crowe, 1999; Poynard et al., 2002; Wessely \& Pariante, 2002). Therefore, as is the case in several other general medical conditions, it is likely that factors other than the disease per se contribute to patients' lived experience of fatigue. Factors that have been studied thus far are presented below.

\section{Anti-viral Treatment}

Interferon (IFN) alpha is a cytokine with anti-viral properties. IFN administered to patients with HCV infection activates an innate immune reaction with increased release of cytokines. The acute physiological reaction to these innate cytokines is a flu-like syndrome with fever, arthralgia, myalgia, headache and anorexia. Subsequently, as patients undergo the standard, long-term treatment, cognitive and behavioural changes may emerge which range from irritability, emotional instability, sleep alterations, cognitive difficulties and psycho-motor slowing to full-blown, clinical depression. However, the most frequent chronic treatment side effect is fatigue which is present in $60-85 \%$ of patients undergoing IFN -alpha treatment (Majer et al., 2008; Nogueria, 2012; Raison et al., 2010; Zeuzem et al., 2000). It has been speculated that innate cytokines induce fatigue by altering the dopamine neuro-transmission in the basal ganglia (Majer, et al., 2008). Fatigue was also predicted by specific changes in sleep architecture, in particular decrease of slow-wave sleep and increase in rapid eye movement sleep (REM) latency in patients with HCV receiving IFN-alpha treatment (Raison, et al., 2010). 


\section{Depression}

The association between depression and fatigue is well documented; however, the prevalence of depression and its contribution to fatigue in the population with chronic HCV infection has not been established. One study, for example did not find any difference between the rate of depression between HCV positive and negative drug abusers (Johnson, Fisher, Fenaughty, \& Theno, 1998). On the other hand, studies conducted with samples recruited from tertiary hepatitis centers show that at least one quarter of patients suffered from current depression symptoms and of patients referred to psychiatric consultation, 58\% were diagnosed with depression (Dwight et al., 2000; Sockalingam et al., 2011; Wessely \& Pariante, 2002). However, the specific settings, the small sample size and low response rate, the cross-sectional design and the lack of control groups raise questions about the validity and generalizability of these results. These studies have confirmed that depression and the severity of fatigue are associated in patients with hepatitis C. One study also showed that depression accounted for one third of variation of fatigue in the sample (Dwight, et al., 2000). However, due to the abovementioned shortcomings of these studies, it is difficult to discern as to what proportion of clinically fatigued patients with HCV infection are depressed and whether fatigue is simply secondary to or comorbid with depression in the HCV population.

\section{Sleep problems}

Only a few studies have examined the prevalence of sleep disorders and their relationship to fatigue in chronic HCV infection (Carlson, Hilsabeck, Barakat, \& Perry, 2010). The evidence generated from these studies indicate that $60-70 \%$ of patients with chronic HCV infection treated in tertiary liver centers complain of low sleep quality and sleep disturbance. There is lack of data on the prevalence of specific sleep disorders (for example sleep apnea, insomnia, restless legs 
syndrome) in the HCV infected population. Of those receiving antiviral treatment, $18-24 \%$ may suffer from insomnia (Zeuzem, et al., 2000). Additionally, limited data suggests high rates of obstructive sleep apnea and daytime sleepiness in patients with liver cirrhosis (Carlson, et al., 2010). The relative contribution of sleep disorders to the fatigue of patients with hepatitis $C$ is unknown. In this context it is notable that approximately $20-50 \%$ of patients with chronic fatigue syndrome have undiagnosed organic sleep disorders, including obstructive sleep apnea, narcolepsy, or restless legs syndrome (Jackson \& Bruck, 2012). Furthermore, 70-95\% of patients with chronic fatigue syndrome struggle with insomnia or have objective sleep features (e.g. low sleep efficiency, prolonged sleep onset latency) that are common in insomnia (Jackson \& Bruck, 2012; Morriss, 1993; Watson et al., 2003). This suggests that undiagnosed sleep disorders may contribute to chronic fatigue of unknown origin.

\section{Illness uncertainty}

Illness uncertainty, defined as difficulty to make sense of illness-related events, have been found to be associated with fatigue over a 12 months period of watchful waiting in a sample of patients with CHV infection (Bailey et al., 2010). Patients experiencing high uncertainty about their illness showed consistently higher level of fatigue during the observation period compared with patients with low and medium levels of illness uncertainty. The authors have postulated that the unpredictability of fatigue and the perception that fatigue is an indicator of disease progression might increase illness uncertainty.

\section{Attributions}

A qualitative study exploring the experience of transition from being healthy to being diagnosed with hepatitis $\mathrm{C}$ infection described that attributions of physical sensations, including fatigue change after the diagnosis. Before having been diagnosed with HCV infection, 
participants attributed fatigue to having small children, ageing, or high workload. The diagnosis gave a new meaning to these experiences; they have been identified and validated as symptoms of a medical illness (Glacken, Kernohan, \& Coates, 2001). Attributing fatigue exclusively to the illness instead of considering other, possible explanations has been described in multiple sclerosis and in insomnia (Morin, Stone, Trinkle, Mercer, \& Remsberg, 1993; Skerrett \& MossMorris, 2006). The possible causal relationship between fatigue attributions and the experience of fatigue has not been explored in the HCV population.

Given that psychological fatigue is an intimately subjective experience that may transform ways of everyday life, it is surprising that only one research report could be located that has explored the characteristics of experienced fatigue among people living with HCV infection (Glacken, Coates, Kernohan, \& Hegarty, 2003). In this qualitative study, participants experienced fatigue as a deleterious condition. Some could recall the exact time of onset whereas other experienced the evolution of fatigue as an insidious process. Fatigue was uniformly described as a multi-dimensional experience encapsulating physical, cognitive, and emotional components. Physical fatigue was experienced as a sense of whole body heaviness, weakness or ache. Cognitive fatigue was predominantly perceived as forgetfulness and emotional fatigue meant experiencing a range of unpleasant emotions, including frustration, anger and anxiety. The trajectory of fatigue fell into two discrete categories: "chronic" and "idiopathic" fatigue. Chronic fatigue was experienced as a permanent mild to severe tiredness. Idiopathic fatigue, on the other hand, was described as a transient, overwhelming sensation perceived as an unpredictable, uncontrollable, paralyzing attack. The description provided by 28 participants in the UK gives insight into the experience of living with HCV infection related fatigue. They shared the multidimensional experience of fatigue with patients of other medical conditions but they also were 
different, as much as they attributed less significance to mental fatigue than to physical fatigue. This is in contrast to reports by patients with multiple sclerosis. Pain, as a feature of physical fatigue also appears to be a phenomenon uniquely presented by this group.

Taken together, clinically significant fatigue is reported by a substantial proportion of patients with chronic HCV infection, independently of the severity of their liver disease. Fatigue is a side-effect of the INF-alpha treatment and is often present when one is clinically depressed. It also may be related to sleep problems, which are frequent complaints of those having a chronic HCV infection. However, the actual prevalence of specific sleep disorders and their contribution to fatigue in this population is unknown. The role of other co-morbidities generally associated with secondary fatigue is unexplored. Preliminary evidence indicates that illness-related cognitive factors, including awareness of having a chronic HCV infection, symptom attributions and uncertainty about the illness might amplify the fatigue experienced by those suffering from HCV infection. The presence or roles of further psychological factors, some of which have already been identified in other patient groups, are yet to be explored.

\section{Study Rationale}

Fatigue is among the leading patient-reported complaints of chronic HCV infection and its treatment. Persistent fatigue may have a significant impact on patients' functional capacity; yet, due to the dearth of research in HCV fatigue in general and the treatment of HCV fatigue in particular, fatigue management is not part of routine clinical practice. Since data generated so far has not shown a causal relationship between hepatitis C virus load and fatigue, identifying modifiable factors that perpetuate the fatigue experienced by individuals with HCV infection is warranted. Preliminary evidence indicates that depression contributes to the fatigue of some individuals with chronic HCV infection. There is also data suggesting that sleep problems may 
be common in this condition and may be related to the fatigue that is ubiquitous in this population. To date, the prevalence of specific sleep disorders and their relative contribution to fatigue in chronic HCV infection has not yet been studied. Also, the behavioural and cognitive patterns associated with fatigue in the context of chronic HCV infection have not yet been systematically explored. The cognitions (for example beliefs about the illness and fatigue and confidence in one's ability to cope with their symptoms) and the actions fueled by these cognitions may have a role in perpetuating fatigue with or without the presence of comorbid sleep and mood disorders. Additionally, patients' beliefs about their fatigue may influence whether and how they disclose it to the health care team. Ultimately, understanding these specific characteristics, triggers and perpetuators of fatigue in hepatitis $\mathrm{C}$ infection can form a basis for the development of fatigue management strategies which may include patient and health care team education, fatigue screening, multi-disciplinary treatment of fatigue-inducing co-morbid conditions, and direct fatigue treatment/management.

This cross-sectional, concurrent mixed methods study explored the characteristics and perpetuating factors of chronic HCV related fatigue. In the study, questionnaires assessed fatigue related functional impairment, sleep disorders (i.e. insomnia and sleep apnea) depression and anxiety symptoms, and fatigue- related cognitive and behavioural factors (e.g. rumination, fatigue perception, and fatigue acceptance). Additionally, the features of fatigue, fatigue management behaviours, beliefs about fatigue and ways in which has fatigue changed patients' functioning were explored using in-depth interviews with patients suffering from moderate and severe, functionally impairing fatigue. 


\section{Theoretical Lens}

The study questions, objectives and design are framed by the scientific theory of critical realism. Critical realism advocates that on the one hand, there is a reality existing independently of our perceptions (ontological realism), while on the other hand our understanding of reality is constructed by mental processes influenced by our personality, identities, social interactions, culture, and situational factors (epistemological reality).

Consistent with the critical-realism framework, the current study acknowledges that fatigue is a real phenomenon (ontological realism) and the experience of fatigue is constructed by the individuals’ perceptions; beliefs about themselves, their illness and fatigue; their social roles and relationships; their personality and lifestyle (epistemological reality). In the particular context of hepatitis $\mathrm{C}$, fatigue is both a physical reality (physiological aspect of fatigue) and a psychological construct (experienced or psychological fatigue). In line with the basic assumptions of critical realism, in order to understand reality, one has to explore both its ontological and epistemological aspects. The former may involve questions concerning the nature of the phenomena that ones’ concepts refer to, whereas the latter focuses on the process of how individual reality is constructed and experienced. The advantage of using a mixed design in the present project was that it allowed the researchers to study the severity and co-morbidities of fatigue (using a quantitative method to explore the ontological aspect of fatigue). At the same time, it made it possible to explore how fatigue is experienced and understood by individuals and the way it shaped their lives (using a qualitative method to explore the epistemological aspects of fatigue).

In addition to being shaped by the perspective of critical realism, the current research project is conceived in the spirit of pragmatism. Pragmatism is concerned with solutions to 
problems and the intent to bring about change. The current study honed-in on a clinical issue, patients' fatigue, and was designed to be able identify modifiable factors that may contribute to fatigue. These factors were assessed both on the quantitative and qualitative components of the study. The qualitative component also included screening for sleep disorders, thus providing the possibility for potential referrals for sleep assessment.

\section{Thesis Hypotheses and Objectives}

Objective 1: Identify the medical, cognitive, and behavioural predictors of fatigue in treatment seeking patients with chronic HCV infection using quantitative methods.

Hypothesis 1: Hierarchical multiple regression analysis will reveal that insomnia, depression, anxiety, fatigue perception, fatigue acceptance, and fatigue-related rumination will significantly predict fatigue above and beyond the severity of liver disease and comorbid medical conditions.

Objective 2: Test the mediation role of specific fatigue- related cognitions and behaviours on the relationship between medical/mental health predictors and hepatitis $C$ related fatigue.

Hypothesis 2: Post hoc exploratory bootstrapping mediation analyses will reveal that fatigue-related cognitions and behaviours mediate the relationship between the medical/mental health predictors and fatigue.

Objective 3: Explore the characteristics of clinically significant fatigue in a sample of patients with chronic HCV infection using qualitative methods.

Hypothesis 3: The qualitative interviews will reveal the perceived course and functional impact of fatigue from the patients' perspective.

Hypothesis 4: The qualitative interviews will reveal general and hepatitis $C$ specific cognitive and behavioural factors related to fatigue. 
Objective 4: Determine if there is an elevated occurrence of insomnia and obstructive sleep apnea in treatment seeking patients with chronic HCV infection.

Hypothesis 5: Screening will identify a greater proportion of patients who: (1) suffer from insomnia, or (2) are at higher risk for obstructive sleep apnea than expected in the general population. Clinically significant insomnia is defined as a score above 14 on the Insomnia Severity Index (ISI). Patients screen positive for obstructive sleep apnea if their scores are at or above 3 on the STOP-BANG Questionnaire.

\section{Methods}

\section{Mixed Methods Approach}

Mixed method research is a strategy of enquiry that combines both quantitative and qualitative approaches. The use of both quantitative and qualitative methods opens the possibility to build on the strength of both approaches and to gain a broader, deeper and richer insight into a specific issue. A further advantage of choosing a mixed method design is that it provides researchers with the freedom to embrace different worldviews and to select the procedures that best serve the purposes of the project. Researchers may use a mixed methods design to expand findings produced first by one method and then to use this information to obtain data by the other method (sequential strategy). For instance, qualitative methods may be used to identify a phenomenon of concern, or to develop a theory which subsequently can be tested and generalized using quantitative methods. An alternative way to use a mixed method design is to integrate qualitative and quantitative findings in order to gain a broad and detailed view of the research topic (concurrent strategy). Finally, researchers may have an overarching theoretical perspective that guides the choice of both quantitative and qualitative strategies (transformative 
mixed method). Within this transformative method, either sequential or concurrent strategies may be used to address the research problem.

The present study employed a concurrent, transformative mixed methods model, using an embedded mixed methods design. In the present project quantitative and qualitative data were collected concurrently at separate research sites. The project was transformational, since the study design and specifically the choice of a mixed method inquiry, the selection of research questions, the specific data collection methods, the data analysis and interpretation of the findings were shaped by the theoretical perspectives of critical realism and pragmatism. The study was designed to generate data that gives a comprehensive view of the psychological fatigue of those with chronic HCV infection with the intent to produce results that have direct clinical utility. To meet this purpose, the research questions prompted the exploration of different aspects of fatigue at different levels of investigation. Specifically, the quantitative component identified some possible medical and psychological predictors of fatigue and explored the mediating role of fatigue-specific cognitions between the medical predictors and fatigue. It also provided a numerical measure of the severity of fatigue-related functional impairment which allows comparisons with other medical populations. The qualitative part was embedded in the quantitative part in that included a sub-sample of participants of the quantitative component, focusing on individuals who had fatigue which caused moderate-severe functional impairment. This subsample was composed of individuals who could provide insight into the phenomenological features of moderate- severe fatigue and who may benefit from fatigue interventions. The inquiry in the qualitative component was on the cognitive and behavioural level of the individual patient. The mixing of the data from the quantitative and qualitative methods was accomplished in the discussion section, when the findings were integrated to 
provide a comprehensive view of fatigue. The advantages of using a concurrent, transformative, embedded method in the research project was that it allowed the exploration of different aspects of fatigue outcomes at different levels. Also, since the study was conducted under the time restrictions of a Master's thesis framework, the concurrent method was a feasible option for data collection.

\section{Participant Characteristics}

A consecutive sample of male and female participants $(\mathrm{N}=132)$ diagnosed with chronic hepatitis C infection was recruited at the University Health Network (Toronto General Hospital) Liver Clinic between October 2012 and May 2013 (Table 4). Individuals were eligible for this study if they were anti-HCV positive verified by ELISA II or III, and confirmed by RIBA II or III, or by PCR for HCV RNA based on their hospital medical records. Only individuals who: (a) had not received antiviral treatment within 6 weeks of enrolment; (b) were over 18 years of age; (c) could read and speak English fluently, without the use of a translator based on their selfreport; and (d) were able to provide informed consent were included in the study. Two individuals were excluded from the study (one did not speak English fluently and the other was not anti-HCV positive). Fifteen participants who did not return the questionnaires were excluded from the analysis. This yielded a response rate of $87 \%$.

The final sample included 115 participants (Table 4). The participants were 23-82 years old $(M=55.82, S D=9.85)$. Almost two-third of the sample (63.85\%) was male. In terms of cultural identities, the majority of participants identified themselves as a Canadian (68.7\%) or European (19.10). Two-third of the participants was married or was in a relationship and $35 \%$ were single. The range of self-reported chronic HCV infection diagnoses ranged from less than one year to 35 years prior to the data collection $(M=12.64, S D=8.95)$. Based on the medical 
records, the sample included participants with the full range of liver disease severity (fibrosis stages F0 to F4). Specifically, 27.7\% of participants had no fibrosis or had mild fibrosis (F0 or F1); $27.7 \%$ had fibrosis of moderate severity (F2 or F3) and $44.6 \%$ had cirrhosis. Almost half of the sample reported a history of anti-HCV treatment (49.6\%); the rest of the sample has not received treatment. Participants reported a history of three comorbid medical conditions on average. Sixty percent of the participants reported that they engaged in regular exercise or did physical work.

Every participant with FSS scores four or above was eligible to participate in the qualitative component. Fifty of the 68 eligible individuals gave consent to be contacted regarding the interview. Four of the 50 potential interview participants did not answer the phone and did not have voicemail services. The remaining 46 participants were invited to participate. Twentyone participants gave informed consent for participation. The main reasons for non-participation were not returning the phone calls or not being available. Of those consented, six participants cancelled the interviews or were not available at the scheduled interview dates. Fifteen interviews were conducted, but one interview was not recorded due to technical problems. The characteristics of the 14 interview participants are found in Table 5. There were significantly more females $\left(\chi^{2}=11.54, p=.001\right)$ and participants who were married or were in a in relationship $\left(\chi^{2}=7.50, p<.01\right)$ in the qualitative sample than in the group of eligible patients with severe fatigue (FSS $\geq 4$ ).

Insert Table 4 here

Insert Table 5 here 


\section{Procedures}

The patients’ physician identified potential participants based on the inclusion criteria and assessed patients’ interest for participation. The physician asked for the permission of interested patients to be approached by the researchers. The study staff explained the study to the interested patients and obtained written consent. Participants completed the questionnaires at the clinic after their appoitment or took the questionnaires home and mailed them to the researchers within 10 days after their appointment. The most recent measure of disease severity was retrieved from the participants' electronic medical records. The diagnosis of HCV infection was also confirmed from the medical records.

During the consent process, participants could also provide a separate, written consent to be contacted by the researchers in the event they were eligible to participate in the qualitative part of the study. Only participants with significant fatigue (Fatigue Severity Scale score at or above 4) were deemed eligible to be selected for participation in the interview. Participants were contacted by phone to assess interest in the participation of the qualitative component. Verbal consent was obtained from those who participated in a phone interview. The interview dates were booked after the informed consent process was completed. Those who opted to participate in an in-person interview were given a description of the interview part of the study during the phone call. These participants also provided written consent in person, before the interview. The interviews were conducted at the Ryerson University Sleep and Depression Laboratory. Each interview was conducted by the investigator of the study. The interviews were digitally audiotaped. Each participant received a \$15 Starbucks gift card as a thank you gift for participating in the interview. 


\section{Sample Size and Power}

The sample size was calculated for the hierarchical regression analysis, since this tested the primary hypothesis. There were no previous studies in hepatitis $\mathrm{C}$ samples with the same measurement tools that this study employed upon which to base the power calculations. Instead, the power analysis was done by setting an a priori desired power level, alpha and the number of predictors, and consulting the literature regarding an appropriate ratio of number of predictors in regression. In order to achieve an adequate power (.8) with eight predictors in the multiple regression model (illness severity, comorbid conditions, DASS depression subscale, DASS anxiety subscale, ISI, DISRS, BIPQ, CFAQ) a sample size between 100 and 150 was required to find a medium size effect (one hundred participants was sufficient for 6 predictors, 150 was sufficient for 10 predictors) (Miles \& Shevlin, 2001). Based on general guidelines, a required minimal sample size is $104+\mathrm{k}$, where k equals the number of predictors in the model (Green, 1991). Based on this guideline, a sample size of $104+8=112$ was necessary for the regression analysis. Finally, calculations using the GPower software set at $\alpha=.05$ with a desired power $=$ .80 and a medium effect size $f=.15$ yielded a required sample size of 109 participants. This equals the sample size (Cohen, 1992) suggested to detect a medium size effect at power $=.80$ and $\alpha=.05$.

\section{Measures, Interviews and Materials}

\section{Measures}

\section{Questionnaires.}

\section{Fatigue Severity Scale.}

The outcome measure of the quantitative component was the Fatigue Severity Scale (FSS). The FSS is one the most popular fatigue scales developed for the assessment of the 
functional outcomes of fatigue (Krupp, et al., 1989a). Respondents indicate their strength of agreement with the nine items of the scale from 1 (strongly disagree) to 7 (strongly agree) referring to the past week or past two weeks. The fatigue score is the mean score of the 9 items. The scale was first validated in lupus erythematosus and multiple sclerosis and showed an internal consistency of .88 and test-retest reliability of .84 (Krupp, et al., 1989a). The FSS has been used in research studies to assess fatigue of patients with multiple sclerosis, cancer, sleep disorders, chronic fatigue syndrome, major depressive disorder, Parkinson’s disease, brain injury, amyothropic lateral sclerosis, and chronic HCV infection (Dittner, Wessely, \& Brown, 2004). The FSS can differentiate between fatigue outcomes reported by samples with major depression, chronic fatigue syndrome and multiple sclerosis (Pepper, Krupp, Friedberg, Doscher, \& Coyle, 1993). The FSS was more strongly associated with the symptoms and the patientreported functional outcomes of chronic fatigue syndrome than the Chalder Fatigue Scale (Taylor, Jason, \& Torres, 2000). Based on the latter findings, it was concluded that the FSS is the more appropriate measure of the functional outcomes of chronic fatigue and of fatigue-related disability (Taylor, et al., 2000). The FSS showed excellent psychometric properties (internal consistency $\alpha=.94$; test-retest reliability during an average of 25 days interval $=.82$, convergent validity with SF-36 vitality subscale $r=-0.76$ ) in a multi-center clinical trial, wherein fatigue was assessed at screening, and baseline before initiation of a combined antiviral treatment in chronic HCV infection (Kleinman et al., 2000). The pre-treatment score was 3.8 in latter sample. It has been suggested that clinical trials use the FSS as a patient reported-outcome measure in chronic HCV infection, because the questionnaire measures functional impairment, it is sensitive to change and it has excellent psychometric properties in this population (Kleinman, et al., 2012). The FSS was chosen as the outcome measure in the thesis because: (a) the construct it assesses 
(the impact/ outcomes of fatigue) was the dependent variable; (b) it has been recommended for use in the HCV population as a patient-reported outcome measure; (c) it has excellent psychometric properties in the HCV population; and (d) it has been validated in a number of chronically ill populations, which allowed the comparison of the mean fatigue score obtained in the present study to fatigue measured in other medical conditions.

\section{Insomnia Severity Index.}

In addition to the fatigue outcome measure, an insomnia questionnaire was also included in the study because fatigue is the leading daytime symptom of insomnia and insomnia is a common disorder in chronic medical conditions (Budhiraja, Roth, Hudgel, Budhiraja, \& Drake, 2011). Limited data also suggests that sleep complaints are common in patients with chronic HCV infection (Carlson, et al., 2010). The Insomnia Severity Index (ISI) is a seven item questionnaire measuring the subjective severity of sleep difficulty; the interference of insomnia with daily functioning; satisfaction with sleep pattern; worry about sleep and daytime symptoms associated with poor sleep (Morin, 1993). The total score is calculated by summing the ratings and higher scores indicate more severe insomnia. Traditionally, the following scores have been used to identify the severity of insomnia: $0-7$ indicates that there is no clinical insomnia; $8-14$ is "sub-threshold" insomnia; 15-21 is moderate insomnia and 22-28 is severe insomnia. Recently, the developer of the questionnaire suggested using a cut-off score of 10 in epidemiological research in the general population and for including patients in clinical trials (Morin, Belleville, Belanger, \& Ivers, 2011). The ISI has adequate internal consistency ( $\alpha=.77)$ and good convergent validity compared with sleep diary; with the sleep onset latency on polysomnography (PSG); and with the global score on the Dysfunctional Attitudes and Beliefs about Sleep scale (Bastien, Vallieres, \& Morin, 2001). An expert consensus has recommended the use of ISI in 
standard insomnia research (Buysse, Ancoli-lsrael, Edinger, Lichstein, \& Morin, 2006). The ISI showed excellent psychometric properties in a sample of cancer patients (internal consistency $\alpha$ $=.90$; test-retest reliability $r=.73-.83$ ), (Savard, Savard, Simard, \& Ivers, 2005). Studies using the ISI in chronic hepatitis C samples could not be located; therefore the psychometric properties of the questionnaire were computed in the present study. Since the lower cut-off score was recommended for use in the general population, the conservative cut-off of 14 were used in the present clinical sample to detect patients with clinically significant moderate-severe insomnia symptoms.

\section{STOP-BANG questionnaire.}

In addition to chronic insomnia, obstructive sleep apnea is also a common sleep disorder (Young, Skatrud, \& Peppard, 2004). A screening tool for obstructive sleep apnea is included in the current study because untreated sleep apnea is associated with daytime fatigue (Tsai \& LeeChiong, 2013). The STOP-BANG questionnaire is a self-administered screening tool for obstructive sleep apnea (Chung et al., 2008). The questionnaire includes seven yes/no questions and a scale where respondents can mark their height and body weight, which is also converted to a positive/negative answer. The questionnaire was validated with pre-surgical patients. A cut-off of three "yes" answers showed a sensitivity of $84 \%, 93 \%$ and $100 \%$ for identifying individuals with apnea-hypopnea indices (AHI) above five, 15 and 30 respectively. The specificity of scores three, four, five, six, seven, eight were $40.3 \%, 60.6 \%$, $79.7 \%$, 91.5\%, $97.5 \%$ and $98.5 \%$ in the pre-surgical population. The cut-off of three was suggested in populations with a high risk for sleep apnea because of the high sensitivity at this cut-off. On the other hand, the cut-off of five is recommended if one wants to achieve high specificity to detect moderate and severe sleep apnea (Chung et al., 2012). The likelihood that one had sleep apnea using three "yes” answers as a cut- 
off was $72 \%-86 \%$ as the scores increased from three to eight, based in the validation study (Chung, et al., 2012). The likelihood that one has a sleep apnea at cut-off five to eight is 77-86 \%. The likelihood of having moderate sleep apnea with scores five to eight was $50-60 \%$. The likelihood that one had severe sleep apnea with scores of 5-8 was 30-38\%. In the current study the distribution of STOP-BANG scores were determined and the the cut-off of 3 "yes" answers was used for screening.

\section{Epworth Sleepiness Scale.}

Sleepiness is the propensity to fall asleep. Fatigue and sleepiness are conceptually separate conditions that are important to be distinguished (for a detailed description, see page 8). A sleepiness measure was included in the study to assess whether subjective excessive daytime sleepiness was a clinically significant problem in the sample. The Epworth Sleepiness Scale (ESS) has been developed to evaluate daytime sleepiness (Johns, 1991). Respondents mark on a scale from zero to three how likely they would fall asleep in eight different everyday life situations. No time-frame is specified. Total scores represent a sum of individual items and range from zero to 24. A score above 10 indicates excessive daytime sleepiness (Johns et al., 1994). The ESS was used to measure excessive daytime sleepiness in a sample of patients with chronic HCV infection referred to psychiatric consultation; the mean score in the sample was 7.4 (SD= 5.76) (Sockalingam, et al., 2011).

\section{Depression Anxiety Stress Scale-21.}

The assessment of depression and anxiety symptoms was important in the thesis because fatigue is listed as a symptom of depression and generalised anxiety disorder in the Diagnostic and Statistical Manual for Mental Disorders, $5^{\text {th }}$ edition (2013). Also, it has been previously suggested that depression may be a contributor to fatigue in chronic HCV infection (Dwight, et 
al., 2000; Sockalingam, et al., 2011; Wessely \& Pariante, 2002). The Depression Anxiety Stress Scale-21 (DASS-21) is a 21 item scale evaluating depression, anxiety, and stress (Antony, Bieling, Cox, Enns, \& Swinson, 1998; Lovibond \& Lovibond, 1995). Respondents rate how they felt over the past week from zero (did not apply to me at all) to three (applied to me very much, or most of the time). The three factors of the scale are: depression (low mood); anxiety (psychophysiological arousal) as well as stress (overlapping tension/irritability components of both depression and anxiety) (Antony, et al., 1998). One study found that the items from the three subscales loaded onto a general negative affect factor, which suggests that the scores can be added to calculate a general negative affect score (Henry \& Crawford, 2005). Scores from each subscale are added and multiplied by two, which yields a total subscale score of zero to 42 . On the depression subscale scores 10-13 indicate mild, 14-20 suggest moderate, 21-27 imply severe, and above 28 imply extremely severe depressed mood. The cut-off scores for anxiety are: 8-9 mild, 10-14 moderate, 15-19 severe, and above 20 is extreme anxiety. On the stress subscale the following cut-off scores indicate increasing degree of stress: 15-18 mild, 19-25 moderate, 26-33 severe, and 34 extremely severe stress. The DASS-21 has good internal consistency (alpha coefficient .94 for depression subscale, .92 for the anxiety subscale and .95 for the stress subscale). The scale shows good construct validity: the correlation between the depression subscale and the BDI was .79; the correlation between the anxiety subscale and the BAI was .85 and the correlation between the stress scale and BDI, STAI-T and BAI were .69, .68, 70 showing that the depression subscale measures depression, the anxiety subscale identifies anxiety and the stress subscale measures the overlapping symptoms of depression and anxiety (Antony, 1998). The DASS-D was chosen to assess depression and anxiety in the present study because the depression subscale does not contain somatic items and the DASS reliably differentiates between 
anxiety and depression (Antony, et al., 1998). There is no available data on the psychometric properties of this questionnaire in the chronic hepatitis $\mathrm{C}$ population.

\section{Brief Illness Perception Questionnaire.}

Three cognitive questionnaires have been included in the thesis, to provide data on fatigue cognitions and behaviours for hypotheses 1 and 2. The Brief Illness Perception Questionnaire (BIPQ) is a short version of the Revised Illness Perception Questionnaire (Broadbent, Petrie, Main, \& Weinman, 2006; Moss-Morris et al., 2002). The BIPQ has nine items, assessing cognitive and emotional components of illness representations based on Leventhal's self-regulatory model (Levethal, 1984). These include beliefs about the identity, timeline, causes, consequences, and comprehensibility of the illness, as well as illness related

personal control, treatment-control, concerns, and emotions. The questionnaire applies a singleitem scale approach for the assessment of each component of illness perception. In the original validation study in a sample of patients with chronic kidney disease the test-retest reliability of the items ranged between .48 and .70 at three weeks and $.42-.75$ at six weeks between the two administrations (Broadbent, et al., 2006). The correlations between the corresponding items of the BIPQ and Revised Illness Perception Questionnaire were between .32 and .63. The low correlation items had moderate correlations with illness specific measures of self-efficacy $(r=$ .47 and $r=.61$ with asthma self-efficacy and diabetes self-efficacy measures). The BIPQ scores discriminated between respondents with diabetes, asthma, minor illness (i.e. colds, allergies, headache), myocardial infarction, and chest pain. The BIPQ was adapted to capture beliefs about fatigue in a clinical trial on the mediators of effect of CBT for fatigue in multiple sclerosis (Knoop, van Kessel, \& Moss-Morris, 2012). In the abbreviated version, two items (causes and identity) were excluded from the questionnaire. A sum score was used for the analysis and lower 
scores indicated a more negative fatigue perception. The psychometric properties of this revised scale were not reported. In the present study five relevant items (concern, coherence, personal control, consequences, emotional representations) were included to assess fatigue- related beliefs. The psychometric properties of the questionnaire were determined in the present study.

\section{Chronic Pain Acceptance Questionnaire.}

The second cognitive -behavioural questionnaire included was an adapted version of the Chronic Pain Acceptance Questionnaire (CPAQ) to assess acceptance of fatigue (McCracken, Vowles, \& Eccleston, 2004). Fatigue acceptance was assessed in the thesis because limited evidence indicates that acceptance may predict changes in fatigue/fatigue outcomes in chronic fatigue syndrome (Brooks, Rimes, \& Chalder, 2011a; Poppe, Petrovic, Vogelaers, \& Crombez, 2013). The CPAQ has 20 items and respondents chose a number from 0 to 6 indicating how frequently the statement is true $(0=$ never true and $6=$ always true). The CPAQ has two subscales: pain willingness (little need to avoid or to control pain) and activities engagement (engaging in normal activities regardless of pain). The total score is calculated from the sum of subscale total scores. The internal consistency of the pain willingness and the activity engagement subscales were $\alpha=.78$ and $\alpha=.82$ in a sample of patients referred to an interdisciplinary pain management program (McCracken, et al., 2004). The scales were significant predictors of pain related disability and distress. The scale was adapted to assess the change in acceptance of fatigue in a cognitive-behavioural therapy (CBT) trial for chronic fatigue syndrome (Brooks, Rimes, \& Chalder, 2011). The researchers used only the "pain willingness” subscale and in the questions they replaced "pain” with “fatigue”. The internal consistency was $\alpha=.83$ in the study. The scale significantly correlated with the total score of the Chalder Fatigue Scale $(r=.241, p<.01)$. In the present study, similar to the above study, the 
word "pain” was substituted with the word "fatigue” and the adjective "chronic” was deleted from the questions. The adapted version is called Fatigue Acceptance Questionnaire. The psychometric properties of the questionnaire are presented in the "quantitative results" section.

\section{Daytime Insomnia Symptom Response Scale.}

The third cognitive - behavioural questionnaire in the thesis was included to assess rumination about fatigue. The Daytime Insomnia Symptom Response Scale (DISRS) assesses symptom focused rumination, defined as repetitive thinking about the daytime symptoms of insomnia, including changes in thoughts and motivation; mood and tiredness (Carney, Harris, Falco, \& Edinger, 2013). The DISRS was validated in a sample of undergraduate students and in adults with comorbid insomnia and depression (Carney, et al., 2013). The construct validity of

the DISRS has been confirmed by: (a) its correlation with measures of insomnia severity, fatigue and general rumination (b) the significantly higher scores in poor sleepers than in good sleepers; (c) the significant, independent predictive value of the DISRS on insomnia severity and (d) separate factor loadings of rumination and worry items (Carney, et al., 2013). The scale had good internal consistency in both the nonclinical and in the insomnia-depression sample $(\alpha=.93$ and .94 respectively). The psychometric properties of the questionnaire were assessed in the current study.

Measure of Illness Severity. According to the Canadian Association for Study of the Liver Consensus Guidelines the degree of liver fibrosis (i.e. deposition of scar tissue in the liver) is the indicator of the severity of liver disease in HCV infection (Meyers, Ramji, Bilodeau, Wong, \& Feld, 2012). The most widely used scoring system validated for use in HCV infection is the METAVIR classification (Bedossa, 2008). According to the METAVIR classification, the stages of fibrosis range from F0 to F4 (F0 = no fibrosis; F1 = portal fibrosis without septa; F2 = 
portal fibrosis with few septa; F3 = portal fibrosis with many septa; and F4 = cirrhosis). In terms of disease severity, F0-F1 is considered none to mild, F2-F3 moderate to advanced fibrosis and F4 as cirrhosis. Fibrosis has traditionally been identified with biopsy. Recently, non-invasive methods have been recommended as frontline assessments of fibrosis in clinical practice (Poynard et al., 2004). FibroTest, for example is a panel of indirect serum markers (Apolipoprotein A1, $\alpha 2$ macroglobulin, haptoglobin, total bilirubin and GGT) that are entered in a formula with demographic variables (age and gender) to calculate a test value that maps into the METAVIR stages of fibrosis as follows: $0-0.19=\mathrm{F} 0 ; 0.2-0.39=\mathrm{F} 1 ; 0.4-0.59=\mathrm{F} 2 ; 0.6-0.79=\mathrm{F} 3$ and $0.8-1=F 4$. The 5 year prognostic value for survival of the FibroTest is similar to the prognostic value of liver biopsy (Poynard et al., 2011). Transient elastography (TE, FibroScan) - an ultrasound based method that has been used for measuring liver stiffness as an indicator of liver fibrosis (Castera, Forns, \& Alberti, 2008). The first validation study of FibroScan in the Canadian population concluded that the major strength of TE from the clinical perspective is the exclusion of F3 fibrosis and cirrhosis (Meyers, Elkashab, Ma, Crotty, \& PomierLayrargues, 2010). The suggested cut-off scores for significant fibrosis (METAVIR equal or larger than F2) range from 5.2kPa-8.6kPa; a meta-analysis based on 50 studies suggested a cutoff of 7.6kPa for fibrosis. (Castera, et al., 2008; Friedrich-Rust et al., 2008). The suggested cutoff scores for diagnosing cirrhosis (F4) are between 9.6 and 14.8kPa; the above mentioned metaanalysis suggested a cut-off of 13kPa (Friedrich-Rust, et al., 2008). The stage of liver disease was retrieved from the most recent medical reports.

Co-morbid conditions, history of previous anti-HCV treatment, and demographic data were obtained from self-reports, as part of the survey. 


\section{Interviews}

A semi-structured interview guide had been developed prior to the qualitative data collection. The interview questions were developed to address objective 3 and hypotheses 3 and 4 of the study (Appendix B). For example, the functional impact of fatigue was assessed using the following questions:

- "What impact has fatigue had on your life?”

- “How does fatigue affect your everyday life?"

- "What are the things you give up doing when you feel fatigued?"

- "What are the things that you can still do when you are fatigued?"

- “How would your life be different if you were less fatigued?”

Follow-up questions asked participants to clarify and elaborate on their answers. Minor changes of the interview questions were implemented after the first interviews. For example, the question whether fatigue affected their infection did not make sense to participants, therefore this question was omitted in the last interviews.

\section{Materials}

The interviews were audio-recorded using an Olympus WS-210S digital voice recorder. A Nexxtech 4318237 telephone recording unit was attached to the audio-recorder to record the phone interviews. SPSS 19 statistical software was used for the quantitative analysis. The bootstrapping mediation analyses were conducted with the PROCESS macro (Hayes, 2012). The qualitative data was coded with NVivo 10 software.

\section{Analysis}


Quantitative Analyses. One participant did not complete the FSS, six participants did not fully complete the CPAQ and four participants had missing items in the DASS. The total scores of these questionnaires were treated as missing data and were excluded from the analyses. Descriptive statistics were calculated to describe the sample characteristics, the distribution of variables, and the survey results (Tables 4, 5, 6, 7, and 8; Figures 3, 4, 5, and 10). Reliability analyses determined the internal consistency of the FSS, ESS, ISI, DISRS, CFAQ and BIPQ questionnaires. The BIPQ was also subjected to a principal component analysis. KolmogorovSmirnov tests were used to determine whether the distribution of variables were significantly different from a normal distribution with the same mean and standard deviation than the sample.

Bivariate, non-parametric correlation tests were used to determine the correlation between variables to inform regression (Table 10). The $\alpha$ levels were set at .05 for the inferential statistical analyses. Bootstrapping multiple hierarchical regression analyses were conducted to test hypothesis 1 (Tables 11 and 12). The bootstrapping sampling method was chosen for the regression because it allowed the precise calculation of the regression statistics from nonnormally distributed samples. The bootstrapping generated the sampling distribution of the regression coefficient by creating 5000 samples from the original sample with replacement and calculated the 95\% bias-corrected accelerated confidence interval of the point estimate of B. Liver disease the severity (stage of fibrosis) and the number of medical conditions reported by the participants were entered in the first block, then ISI, DASS-depression and DASS- anxiety in the second block and finally DISRS, CFAC, and BIPQ were entered in the third block in the regression model.

Exploratory bootstrapping mediation analyses tested whether fatigue related cognitions mediate the effect of medical conditions on fatigue (Figures 6, 7, 8, 9 and Table 13). The 
bootstrapping method was chosen for the mediation analyses because: (a) it allows the inclusion of multiple mediation pathways, while eliminating the issue of estimation bias that occurs when several mediators are tested individually in simple mediation models; (b) it calculates the free-ofscale effect size for each mediator, thus these effect sizes are directly comparable and (c) can calculate indirect effect even when there is not direct (non-mediated) effect between two variables.

Insert Figure 6 here

The frequency distribution of the ISI and the STOP-BANG questionnaires were used to inform hypothesis 5 (Table 7, and Figures 5, 10). Additionally, a one-way ANOVA and KruskalWallis analysis tested whether there were significant differences in FSS and ESS scores between groups with moderate/severe clinical insomnia and possible moderate/severe sleep apnea (Table 14).

Qualitative analysis. The interviews were audio-taped and transcribed verbatim. The analysis of the qualitative data used a template coding method. Initial code manuals had been developed prior to the data collection. These coding templates have been derived from the researchers' clinical experience and from existing literature on fatigue in medical conditions (Appendix C). The main themes in the initial draft code books were also incorporated into the main sections and prompts of the interview guide (Appendix B). The data analysis started when two-third of the qualitative data had been collected. As the first step of the analysis, the transcribed interviews were read several times both vertically (individual interviews from beginning to end) and horizontally (identical sections across interviews). In line with the 
pragmatic perspective of the research project, the pre-existing code manuals were modified in this initial, reading stage and after the coding of the first interviews. Next, the transcribed interviews were coded by the principal coder using these manuals. The data collection was completed at saturation (the existing codes captured the relevant content of the interviews and subsequent interviews did necessitate changes in the existing themes) (Kelly, 2013). A reliability coder (PhD candidate) coded 100\% of the interviews. The training of the reliability coder took place on one interview. Subsequent to the training, the reliability coder analyzed $50 \%$ of the interviews and the coders met to discuss the results. The principal coder made refinements in the coding instructions based on the discussion. Next, the coders independently coded the remaining data. A next meeting took place after $100 \%$ of the interviews had been coded. The discrepancies between the results were discussed and the items were recoded according to the consensus between the coders. In rare situations, wherein a consensus was not easily achieved, the coders did not make changes in the coding. This ensured the autonomy of the coders and the left space for alternative interpretations within the existing coding framework. Inter-coder reliability was determined as the percentage agreement between coders per each sub-category across participants. The final average inter-rater reliability for the whole sample was $97.7 \%$ (86-100\%).

Mixed method analysis. The BIPQ "causes" item asked participants to rank-order the three most important factors that they believe caused their fatigue. Participants were free to write the perceived causes of their fatigue (i.e. this was not a forced choice item). The fatigue attributions generated by the whole sample were first categorized and sorted (qualitative analysis) then the attributions of the high fatigue $(\mathrm{FSS} \geq 4$ ) and the low fatigue (FSS $<4$ ) groups of the full sample were compared using quantitative methods. Chi-square test compared whether 
there was an association between fatigue (high versus low) and attribution categories. The effect size (odds ratio) was calculated for the significant effects (Table 16).

\section{Results}

\section{Quantitative Results}

Below is the description of the statistical results based on the survey data. This section contains the descriptive statistics of the data. It also presents the statistical test of the models pertaining to the relationship between the medical, psychiatric and cognitive predictors and fatigue.

Preliminary analyses. Tables 6, 7, and 8 and Figures 3, 4, and 5 display the descriptive statistics of the study variables and survey results. The descriptive statistics presented in Table 6 were calculated using bootstrapping to provide accurate estimations of the population parameters.

Fatigue and sleepiness. The FSS scores ranged from 1 to 7 (Table 6). The distribution of the FSS was negatively skewed. The median FSS score was 4.67 (interquartile range = 2.92). The scale showed excellent internal consistency in this study (Cronbach's $\alpha=.94)$. Almost $60 \%$ of the total sample (56.9\% of males and $64.3 \%$ of females) obtained a score indicating moderate or severe fatigue - related functional impairment (FSS $\geq 4)$.

The median ESS score was 9.00 (interquartile range = 8). The questionnaire had good reliability (Cronbach's $\alpha=.83$ ). Almost half of the sample experienced excessive daytime sleepiness based on their ESS scores (ESS $\geq 10$ ). There was a significant correlation between fatigue and sleepiness (Spearman's rho $=.51, p<.001$ ). Based on the cut-off scores for excessive daytime sleepiness (ESS $\geq 10$ ) and moderate-severe fatigue (FSS $\geq 4$ ) the sample could be divided into four groups (Figure 3): those without significant sleepiness and fatigue (26.8\%); 
participants with only fatigue but no excessive daytime sleepiness (26.8\%); those with excessive daytime sleepiness and normal fatigue (12.5\%); and those with both excessive daytime sleepiness and fatigue (33.9\%). Figure 4 displays the results of the survey question on the possible benefits of a fatigue treatment/management service at the liver clinic.

Insert Table 6 here

Insert Figure 3 here

Insert Figure 4 here

Symptom questionnaires. Tables 7 and 8 display the distribution of the symptom questionnaire scores. The ISI scores were normally distributed. The mean ISI score was 11.59 $(S D=7.76)$, which is in the range of "sub threshold" insomnia and is above the cut-off suggested for use in epidemiological studies in the general population (Bastien, et al., 2001; Morin, et al., 2011). The questionnaire had excellent internal consistency $(\alpha=.92)$. Figure 5 shows the distribution of the STOP-BANG scores in the sample. The scores ranged from 0 to 8 . The median score was 3 and the mean was 3.6, which are at/above the suggested cut-off to detect possible sleep apnea cases with high sensitivity (Chung, et al., 2012). The majority of participants had DASS depression and anxiety scores in the normal range (DASS-D $=82 \%$, 
DASS-A = $80.9 \%$ ). The scale had high internal consistency (depression subscale Cronbach's $\alpha$ $=.89$; anxiety subscale Cronbach's $\alpha=.82$; stress subscale Cronbach's $\alpha=89$ ).

Insert Table 7 here

Insert Table 8 here

Insert Figure 5 here

Cognitive-behavioural questionnaires. The DISRS had a normal distribution with a mean of $41.97(S D=15.63)$, which is similar to the mean score of the non-clinical validation sample (41.43, $S D=11.42$ ). The internal consistency of the questionnaire was excellent ( $\alpha=$ .97). Both CFAQ subscales showed good reliability (CFAQ-activities engagement subscale $\alpha=$ .85; the CFAQ- fatigue willingness subscale $\alpha=.82$; CFAQ whole scale $\alpha=.92$ ). The mean of the fatigue willingness subscale in the current study $(M=27.60$, bias corrected $95 \%$ confidence interval $=24.95-30.07, S D=13.58)$ was lower than it was in a sample of patients with chronic fatigue syndrome $(M=34.86, \mathrm{SD}=9.56)$ indicating a higher acceptance of fatigue (less avoidance and less need to control the fatigue) in the current sample (Brooks, Rimes, \& Chalder, 2011).

Each BIPQ scale represents one dimension of illness perception in Leventhal's selfregulation model (Levethal, 1984). It has been recommended that the items are used 
individually; alternatively, a composite score can be calculated with higher scores indicating a more threatening view of the illness (Sivertsen, 2013). Based on the scoring instructions, the internal consistency of the questionnaire may differ across illnesses (Sivertsen, 2013). One previous study has included the BIPQ to assess fatigue perception (Knoop, et al., 2012). In this study two items were omitted and the composite score was used to represent how threating the fatigue was perceived by the participants. Since both the composite score and the individual items can (and have been) used and the present study included only selected items, an exploratory factor analysis was conducted before the main analyses. The questionnaire had adequate reliability (Cronbach's $\alpha=.76$ ). However, the questionnaire item "coherence” (i.e. "How well do you understand your fatigue") had very low correlation with the total score ( $\alpha=$ .08). Deleting this item increased the overall reliability to Cronbach's $\alpha=.85$. Additionally, this item had lower than .3 correlations with the other four items. Therefore the "coherence" item was excluded from the factor analysis (Field, 2009). A principal component analysis (PCA) with orthogonal (varimax) rotations was conducted on the items "concern”, "consequences", "control” and "emotional representations". The Kaiser-Meyer-Olkin measure confirmed that the sample size was adequate for PCA, $K M O=.78$. The $\mathrm{KMO}$ values for the retained individual items were between .77 and .93 which are significantly higher than the acceptable threshold of .5 (Field, 2009). The Barlett's test of sphericity was significant $\chi^{2}(6)=238.358, \mathrm{p}<.001$ indicating that the correlation between the items were adequate for PCA. The PCA revealed that only one component had an eigenvalue above the Kaiser's criterion of one. This single "fatigue perception” factor explained $69.77 \%$ of the variance. The factor loadings were as follows: concern .91; consequences .90; emotional representations .89; and control .61 (Table 9). Given that the items loaded into a single "fatigue perception" factor and the scale showed a high 
internal consistency, the total score of relevant items were appropriate to be used in the current study. From a theoretical perspective, it appears that there may be an overlap between the BIPQ “consequences" item and the FSS as both elicit information about the perceived consequences of fatigue. In order to test a theoretically sound regression model (i.e. to avoid a possible conceptual overlap between a predictor and the outcome), the "consequences" item was not included in the total BIPQ score in the regression analyses. The BIPQ including the items "concern”, "control” and “emotional representations” had a good internal consistency (Cronbach's $\alpha=.80$ ).

According to the Kolmogorov-Smirnov test, the ISI, DISRS, and the CFAQ scores were normally distributed. The FSS scores were reversed and then a square root transformation was performed to eliminate the negative skewness of the distribution. On the other hand, the DASSD, DASS-A and BIPQ total scores were not normally distributed, even after transformations. Therefore, a non-parametric statistic (Spearman's rho) was chosen to test correlations between the relevant variables and bootstrapping was used to determine the distribution of descriptive statistics and regression coefficients. The non-transformed FSS was used in these non-parametric tests.

Insert Table 9 here

\section{Main analyses.}

Exploratory correlations. Table 10 displays the correlation coefficients of the relevant study variables. The correlation between the FSS and the degree of liver fibrosis was low ( $r$ ho $=$ $.13, p=.10)$. The FSS had the strongest correlations with the BIPQ (rho $=.75, p<.01)$, in particular with "concern about fatigue" ( $r h o=.74, p<.01)$. Further, the FSS was moderately 
correlated with other fatigue-specific cognitive and behavioural questionnaires, in particular with the BIPQ emotional representations (rho $=.66, p<.01)$; the DISRS $(r h o=.64, p<.01)$ and CFAQ $(r h o=-.57, p<.01)$. In addition, there were moderate correlations between the FSS and the ISI ( $r h o=.51, p<.01)$ and the FSS and the DASS-depression subscale $(r h o=.51, p<.01)$.

\section{Insert Table 10 here}

Hypothesis 1 stated that insomnia symptom severity, depressed mood, anxiety, fatigue perception, fatigue acceptance, and fatigue-related rumination would significantly predict fatigue above and beyond illness severity and medical comorbidities. Hierarchical linear regression analyses based on 5000 bootstrap samples tested this hypothesis.

The degree of liver fibrosis and the number of other medical conditions were entered in the first block to test the predictive value of the liver disease and comorbid medical conditions on fatigue. This initial model significantly predicted fatigue, accounting for $5.4 \%$ variance in the FSS scores $F(2,98)=3.85, p<.05$, adjusted $R^{2}=.054$ (Table 11). The bootstrap bias corrected accelerated 95\% confidence intervals of the regression coefficients indicate that only the number of other medical conditions (excluding fibrosis) contributed significantly to the model. The ISI, the DASS depression and DASS anxiety were added to the model in the second step to test whether insomnia, depression and anxiety symptoms predict fatigue. The ISI and the DASS depression scores significantly improved the model, accounting for an additional 23\% variation in the fatigue scores $F(3,95)=8.95, p<.001$, adjusted $R^{2}=.284$. Finally, the cognitive variables (DISRS, CFAQ and BIPQ) were included in the third block. The inclusion of the cognitive-behavioural variables increased the predictive value of the model by $24 \%$. The final model accounted for $52 \%$ of the variation in fatigue $F(3,92)=14.57, p<.001$, adjusted $R^{2}=$ 
.521. The bootstrap bias corrected accelerated 95\% confidence intervals of the regression coefficients indicate that the only cognitive variable that contributed significantly to the final model was the BIPQ.

After having established which variables contributed significantly to the initial model, a second regression analysis was run with the significantly contributing variables according to (Field, 2009) (Table 12). Specifically, the medical conditions (total number of medical comorbidities, depression, and insomnia) were entered in the first step and the BIPQ was entered in the second step. The "medical block" explained $30.4 \%$ of the variation in fatigue and was accounted for by the significant contribution of the DASS-D and the ISI to the model, $F(3,103)$ $=16.41, p<.001$, adjusted $R^{2}=.30$. Adding the illness perception improved the predictive value of the model by an additional $18 \%$. Thus, the final model accounted for $48.4 \%$ of the variation in the FSS, $F(5,101)=25.87, p<.001$, adjusted $R^{2}=.48$. The symptoms of depression as well as the BIPQ made statistically significant contributions to the final model.

The individual contribution of the illness representation domains were examined by entering the three constituents of the BIPQ separately in the third block. This model shows that the emotional and cognitive symptoms of depression (DASS-D) as well as the "concern" and "control” domains of illness representations (but not the BIPQ emotional representations domain) were the significant predictors of fatigue related functional impairment in the final model. The above model was the best fit of the data for the whole sample and for both sexes separately.

Insert Table 11 here 
Insert Table 12 here

Test of hypothesis 2. Post hoc bootstrapping mediation analyses explored whether the effect of medical conditions on fatigue was mediated by fatigue-specific cognitions and behaviours. In the regression model only the IBQ concern and control domains predicted fatigue therefore only these cognitive items were included as mediators in the mediation models. A general mediation model is depicted in Figure 6. The specific models described below are displayed in Figure 7-9.

The first model examined the effect of depression on fatigue (Table 13 and Figure 7). The total effect of DASS-D on FSS was significant (effect $=.175, S E=.033, p<.001)$. Based on the model, the DASS-D exerted its effect on the FSS through two paths: a significant direct effect $($ effect $=.065, S E=.026, p<.05)$ and a significant indirect effect through the cognitive mediators (effect $=.109, S E=.024, p<.001)$. Comparing of the individual effect sizes of the two illness representation domains revealed that the effect of the BIPQ concern (effect $=.091, S E=.024$, bias corrected 95\% interval $=.050-.142)$ was larger than the BIPQ personal control (effect $=$ $.018, S E=.012$, bias corrected 95\% interval $=.001-0.051)$.

The second model explored whether fatigue representations (i.e. BIPQ concern and control items) mediated the relationship between insomnia (ISI) and fatigue related functional impairment (FSS). In this model, insomnia significantly predicted fatigue (effect = .115, $S E=$ $.177, p<.001)$. Based on the model, insomnia exerted its effect on fatigue primarily through the fatigue representations, indicated by the significant indirect effect paths. On the other hand, the direct effect of ISI on FSS was not significant. The magnitude of the indirect effect of the ISI on 
FSS was larger through the BIPQ concern about fatigue (effect $=.082, S E=.152$, bias corrected $95 \%$ interval $=.054-.113)$ than it was through the BIPQ personal control item (effect $=.020, S E$

$=.009$, bias corrected $95 \%$ interval $=.006-.045)$.

The third model tested the effect of the medical comorbidities on FSS (Table 13 and Figure 9). The comorbid medical conditions (excluding fibrosis) had both a significant direct and indirect effect on fatigue. The indirect effect was mediated only by the IBQ-concern item.

Insert Figure 6 here

Insert Figure 7 here

Insert Figure 8 here

Insert Figure 9 here

Insert Table 13 here

Hypothesis 3 and 4. The characteristics of fatigue; fatigue cognitions; fatigue management behaviours and the functional impact of fatigue are described in the qualitative 
results section below.

Hypothesis 5 - Insomnia and obstructive sleep apnea statistics. More than third of the sample (30.1\% of males and $43 \%$ of females) scored above the cut-off indicating moderate or severe clinical insomnia (Table 7). Figure 5 displays that $71 \%$ of the sample $(80.9 \%$ of males and $54.8 \%$ of females) had scores suggestive of obstructive sleep apnea (i.e. scores at or above the cut-off score of 3 on the STOP-BANG questionnaire). The STOP-BANG score 5 and above indicating high risk for moderate or severe sleep apnea were obtained by $29.5 \%$ of the sample (41\% of males and $9.5 \%$ of females). Sixty two percent of the individuals with scores STOPBANG $\geq 5$ also had subjective excessive daytime sleepiness. Only $7 \%$ of the total sample had been diagnosed with sleep apnea.

The sample was divided into four groups based on the cut-off scores for moderate-severe clinical insomnia (ISI $>14$ ) and obstructive sleep apnea (STOP-BANG $\geq 5$ ): $49.6 \%$ did not endorse symptoms suggestive of clinically significant OSA or insomnia; $20.9 \%$ had probable clinical insomnia only; $14.8 \%$ had probable OSA only; and $14.8 \%$ scored above the cut-off for both moderate-severe clinical insomnia and OSA (Figure 10). A one way ANOVA showed that there was a significant statistical difference between the above groups in fatigue $F(3,109)=7.79$, $p<.001$ (Table 14). Post hoc tests indicated that participants with clinical insomnia only and those with a combination of clinical insomnia and potentially moderate/severe sleep apnea reported significantly higher fatigue than those without a clinically significant sleep problem or with a possible sleep apnea only (Table 14). Since the ESS scores were not normally distributed in the non-sleep disordered group, a Kruskal-Wallis test determined that there was a significant difference in sleepiness across the four groups. A one-way ANOVA did not show significant differences in sleepiness across the three groups reporting symptoms of sleep disorders, 
$F(3,109)=4.86, p<.05$, but post-hoc tests indicated that those who scored above the cut-off on both the insomnia and sleep apnea questionnaires had significantly higher scores on the ESS than those who did not report clinically significant sleep issues. The mean ESS was above the cut-off for excessive daytime sleepiness in the group with insomnia and in the group with the combination of clinical insomnia and sleep apnea (Table 14).

Insert Table 14 here

Insert Figure 10 here

\section{Qualitative Results}

The results of the interview data are presented below. Fourteen interview transcripts were analysed. The final coding book included eight main themes and 37 sub-categories. The mean word length of the interviews was 7994 words. The main themes and the sub-categories within these themes are described below with pertinent quotes to highlight the categories. The main themes are: characteristics of fatigue; the course of fatigue; triggers of fatigue; fatigue-related cognitions; fatigue-management behaviours; the functional impact of fatigue, communication about fatigue with health care providers and reasons for not communicating about fatigue with health care providers. The main themes and categories are also displayed in Table 15.

Insert Table 15 here 
Characteristics of fatigue. The following themes emerged from participants' description of their fatigue: "physical”, "cognitive”, "emotion as a consequence”, "persistent” "starts early”, and "sudden, complete exhaustion". Seventy two percent of participants described their fatigue as an exclusively, predominantly or primarily a physical experience. Some participants also identified a cognitive aspect of fatigue, such as an impaired ability to focus or concentrate. Participants reported that physical and cognitive fatigue may cause emotional changes that are experienced as sadness, irritability, or "feeling down”. Participants stated that these emotions occurred after the physical sensation of fatigue had started or that they were the direct consequences of fatigue. Participants reported that their fatigue may be different from normal fatigue because it is persistently present every day or most days without any apparent trigger and because it starts early in the day. Their understanding was that "normal" fatigue starts later in the day and is a consequence of "being worn down". Some reported that they experienced fatigue as a sudden wave of complete, debilitating exhaustion.

Course of fatigue. The categories of "chronic stable course" and "chronic progressive course” emerged within this main theme. Every participant indicated that their fatigue had a chronic course. Their perception was that it changed from normal to unusual or had become problematic months or years prior to the interview. The participants' descriptions revealed two types of chronic fatigue: some reported that their problematic fatigue level has been overall stable over the course of months/years (chronic stable course), whereas others described that their fatigue progressively became worse (chronic progressive course).

Triggers of fatigue. Participants identified the following factors that exacerbated their fatigue: activity, inadequate sleep, stress, and HCV-related anxiety. Seventy eight percent of participants described that their fatigue was exacerbated by activities. The key activities 
provoking fatigue were those that involve movement including: chores; walking to the neighbours; bending; exercise; and physical work. Some noted that routine, light activities, for example bending, walking at short distance, or chores triggered extreme fatigue. Some also mentioned that sustained mental activity triggered their fatigue. In addition to the effect of particular types of activities, participants also discussed that an imbalance between one's energy and the amount, duration, or intensity of activities ("overdoing” or "pushing the envelope too hard”) exacerbated fatigue.

Fifty-seven percent of participants noted that disrupted sleep may have triggered their fatigue. Stress related to family issues, social relationships, work, living circumstances, and finances was also identified as a trigger of fatigue by $43 \%$ of the sample. Uncertainty and anxiety about the prognosis of the HCV infection and the threat of a foreshortened future were mentioned in the context of exacerbated fatigue by $36 \%$ of participants. Representative quotes are presented below.

There’s concern there. I mean I always get upset to some minor degree when I go down every six months for my appointments and I worry in advance because you know the day will come and they’ve basically told me this, that if I - when I come if they do the test and if there's liver cancer. You learn to manage it like you have to manage; everyone has to manage their stress. But it is stressful you can say, call it what you like. It is; it is stressful.

You know I get anxious when I am waiting for the news; I'm waiting for information from the doctor. Or I get a phone call from the doctor and it’s like, everything starts moving so fast and I get overwhelmed and that drags me out. 
Fatigue-related cognitions. "Fatigue attributions”, “control”, "prognosis”, “acceptance” "positive attitude” and "cognitive avoidance” emerged as fatigue-related cognitive themes. Participants attributed their fatigue to the HCV infection, to other medical conditions, or to a chronic sleep problem/sleep disorder. Eighty six percent of participants shared their beliefs that their HCV infection has caused their fatigue. Some of these participants discussed that they attributed their fatigue to the HCV infection because they were otherwise healthy and led a healthy lifestyle.

I think it's from hepatitis C, it has to be the virus in me. Where else is it coming from? I'm not taking any drugs of any kind I'm not on any medication. I don't drink alcohol. I'm not eating bad food, I don't drink coffee... So you tell me where it's coming from. It can’t be because something happening, this is dragging me down, it just happens. Another participant reasoned:

I'm not overweight. I have no blood pressure problems, I'm not diabetic. I have nothing. None of, I have no illnesses whatsoever other than hep C. That's the only problem I have and that's the only thing that's doing this to my body.

Others attributed their fatigue to the virus because of the temporal relationship between the onset of the fatigue problem and the diagnosis of the infection.

"The reason that I did attribute it to hep C was that... when I was diagnosed it was the fatigue that took me to the doctors."

Another participant explained:

"Well it seems to be the only thing that changes. Like I got this liver disease and then all of a sudden I started feeling worse. I didn’t get anything else that can explain it.” Thirty six percent of participants attributed their fatigue to a medical condition other than 
hepatitis C. These conditions included urological problems, diabetes, high hemoglobin, and fibromyalgia. Of these participants, two believed that their fatigue was also caused by a chronic sleep problem. An additional participant who did not have another chronic medical condition attributed her fatigue to her poor sleep.

Seventy-nine percent of the sample shared their thoughts about their control over fatigue. One third of these individuals reported some degree of control over their fatigue or over the functional impact of fatigue. In this group, 57\% of participants discussed that balanced activities and a regular schedule helped them to keep their fatigue under control.

I think I can regulate it in the sense that I know I'm limited with what I can do so I don't try to push myself before I get started. I say I'm going to do this much, this much I feel like I accomplish and I can. And so it allows me to be in control rather than let the situation control me.

The other $43 \%$ of the group described that they could manage their fatigue by going for a disability, sick leave, or part time work, as they had more freedom to schedule their activities and they could rest whenever they needed to.

I just can deal with it but I don’t know I can handle it. Like I said the only way I can handle it is to, that I haven't had to go into work and I don't have to do long stretch of anything.

Others reported that they did not believe that they had any control over their fatigue.

Seventy-nine percent of participants shared their beliefs about the prognosis of their fatigue. Fifty-five percent of these individuals predicted that their fatigue would worsen as they aged and their liver disease progressed. Twenty-seven percent predicted their fatigue would not worsen as long as they maintained their current fatigue management strategies (activity 
scheduling or staying at home). Eighteen percent hoped that their fatigue would decrease if their liver disease was effectively cured.

The participants' descriptions revealed three main cognitive coping strategies:

acceptance, cognitive avoidance and positive attitude. Sixty four percent of participants indicated that they had accepted chronic fatigue as part of their lives, had come to peace with this situation, and had learned how to live with it.

"I’ve just learned to just roll with what life sends me... it’s basically you know I think, you know we have to, all of us have to be able to cope and adjust.”

"I think I’ve accepted my reality and I think that's half the battle right?”

Descriptions of cognitive avoidance were found in $43 \%$ of the sample. These participants reported that they tried to avoid thinking about their fatigue because it was not helpful and could make things worse.

If you think about how sick you are, if you think about how tired you are, if you think about how angry you are you're going to stay in that component of thought. And so you have to learn to distract yourself.

"So out of mind, out of sight out of mind you know what I mean, like I know I'm trying not to think of it.”

The importance of positive attitude in coping with fatigue was discussed by fifty seven percent of participants.

I'm trying to do my best and I'm a strong man, you didn't see me, you don't meet me but I'm a strong man as I mentioned, I did a lot of sports. I have to go on for my kids... I am trying to you know as strong as I can and I am forcing myself to be strong. 
“I try to keep as positive as I can. That’s my way of trying to cope with it. I would see it as a disability. It doesn't have to stop you from getting to your goals.”

“And you might as well enjoy and live your life, because it won’t change by “boo hooing”, will it? You make yourself worse by doing that so be positive and be strong and be happy.”

Fatigue-management behaviours. Participants engaged in the following behaviours to manage their fatigue: non- active rest; sleep; active rest; physical activities; nutrition and pleasurable activities. Two additional fatigue-related behavioural patterns could be identified based on the participants' descriptions: fatigue driven activity and pacing.

Seventy two percent of participants reported non-active rest as a behavioural response to fatigue. These participants rested without falling asleep or engaging in other activities. Although non-active rest was the most frequent coping behaviour in the sample, only one-third of those engaging in this coping behaviour reported that non-active rest actually alleviated their fatigue. Sixty four percent of the sample described that they slept as much as possible during the night or they take short naps to manage their fatigue. Half of this group reported that they got a burst of energy after a good sleep. Fifty seven percent of participants reported that they used active rest when they felt fatigued. During active rest, they sat or lied down while they actively engaged in non-physical activities for example reading, paperwork, watching an interesting program on the TV, knitting, or using the computer for checking their e-mails, social networking, or surfing on the internet. Participants also discussed nutrition as a fatigue management behaviour. Some described that they followed a healthy diet and took vitamins or food supplements to combat their fatigue. Others emphasized the importance of eating and proper hydration to prevent exhaustion. 
Although I'm really, really tired, I'm just aching to sit down, I know I have to eat, I have to make myself something to eat because I will sit down and in half an hour I will start to feel really sick as well as feeling really tired.

One participant reported a habit of eating carbohydrate-rich food when feeling fatigued whereas another participant avoided food containing processed sugar because "I find that I get really hyper and then I crash.” Someone found eating protein-rich foods helpful. Two participants mentioned that they drank coffee when feeling fatigued. In terms of the effectiveness of these behaviours, some reported that healthy diet was instrumental in preventing their exhaustion. Others described that they felt fatigued despite following a healthy diet.

"I also changed my diet. I had this idea that if I ate really well and took all these supplements, I'm on an anti-inflammatory diet. And that's super-duper healthy, but I feel like crap.”

Some participants mentioned that they tried to engage in pleasurable activities to prevent or to combat fatigue.

Overall participants described two main activity patterns: fatigue driven activities and pacing. Fatigue driven activities were "reactive” behavioural responses to fatigue. The fatigue driven coping patterns included frequent or excessive rest alternating with bursts of activities in periods when one feels less tired; cancelling activities because of fatigue; and making decisions on activities in the moment based on current fatigue level.

“The fatigue definitely is: How do I feel at this moment? It’s a day to day thing. I do make the plans and of course when that time comes if I can't do it, I just don't do it.”

"Most physical stuff that I do is the house cleaning and I kind of do it in little spurts when I’m feeling okay.” 
The second activity pattern reported by the participants was proactive pacing. Those who used pacing described that they budgeted their energy to last for the whole day; made activity plans in order to avoid over-exertion; broke up activities into small parts; scheduled rests between activities and adapted the intensity of their activities to their level of fatigue.

I make out a list every week for the whole week of what I'm going to do this day, the next day, the following day. If I'm tired today then I'll do less. If I'm fatigued, the same, you know the same thing.

"Rather than just rushing through something, doing it at a slower pace my energy levels tend to stay a little longer so I'm able to accomplish more even though I'm working a little slower.”

Over the course of this, especially now that it's increasing, I'm a firm believer that it's like a bank account that I can deplete. And if I spend too much then I'm taking from tomorrow. Well if there's three things that need to get done, instead of worrying about plowing through one of them all the way from start to finish knowing that's going to exhaust the crap out of me, I'll work a bit on all three or I'll tell myself “you've got Monday and Tuesday to do it”. But I tend, I do try and structure in these breaks... usually work for about 45 minutes, an hour and then I'll get up, wander, go get a glass of water or something. It also helps me become aware of “Okay, how long do you still have?”; “How far have you gotten?”; “Oh this is more than you thought it would be”. That's another thing that is, to check with myself, “Okay you thought this would take two hours, you've been at it for an hour, you're one third through, okay stop now, work on it again tomorrow because today you also have to do this and this. 
Functional impact of fatigue. Participants reported that fatigue has caused impaired functioning across multiple life domains, including "family life and intimate relationships", “physical functioning”, “work”, “social life”, “active recreation” "finances”, and with respect to “future plans”.

Seventy-two percent of participants reported that family life, including family dynamics and roles in the family, the activities that the family engages as well as participants' intimate relationships or relationship status have been affected by fatigue. Participants discussed their struggle to maintain their self-esteem and their work contribution to the family. They also described the emotional impact of their fatigue in the context of family dynamics.

Rob $^{1}$ and I are still in love. You know nothing’s going to change that but like, my husband and I have never had a knock down drag out fight. We have never had a major argument. But I’m finding that sometimes he's trying too hard and that makes for a tense situation too. I'll try to do it and I knock myself out kind of thing... and then he gets upset with me because I overdid it.

Another participant decribed:

They try to be supportive but I know my son's worried about my health and that and he tried to get me motivated in going to the gym or doing stuff. My wife's the same way but I mean you know it’s frustrating on her because like I said our house has been a construction zone for some time now and it's grating on her... and she'll say to me: Hire somebody to do it. No I don’t wanna hire somebody I want to do it myself. You know and that kind of stuff and I know she's thinking of everything like she's thinking of me and she's thinking of what needs to be done. But I'm a stubborn man and I don't like to admit what I can’t do.

\footnotetext{
${ }^{1}$ The name was changed to protect confidentiality.
} 
Twenty-one percent of participants shared their beliefs that they did not have intimate relationships or were single because of their fatigue.

I was in a relationship where you know, he was one of those people that wanted to go twenty four-seven and I couldn’t keep up, I just couldn’t keep up. There was never any peace and I was really suffering physically for it and, in truth, I feel that that relationship ended because I was exhausted. And he had issues but from the physical perspective for me I just felt exhausted like I just couldn’t physically live that way anymore.

Another participant commented is follows:

And the fatigue... without a doubt you know. My marriage failed because of that and most of my relationships do. Again I would say it's like a disability because you can't be intimate with a man if you're feeling really fatigued. You know the last thing on my mind... it’s like "Oh go away” you know.

In terms of physical functioning, participants described an impaired ability to engage in physical activities, for example walking to places or doing chores. Participants described that they had given up some of these activities or they did less and at a slower pace than they had used to. Sixty four percent of the sample reported that their ability to work had been significantly affected by fatigue. For some, fatigue contributed to significant changes in their work status: they went on disability; could not go back to work from disability; gave up an active volunteer life altogether or had been on sick leave for a year at the time of the interview because of their fatigue. Others reported that they worked only part time to be able to manage their fatigue. Some of those working described that they became exhausted at work and as a consequence, they were almost dysfunctional at home after work. Others explained that they set limits in terms of work hours per day or per week and it helped them to manage their fatigue. 
Sixty four percent of participants described that fatigue affected their social lives in that they went out less, visited their friends less frequently, spent shorter time with their friends, were able to engage only in sedentary activities when they spent time with their friends and invited their friends less frequently than they used to because of their fatigue. Half of the sample reported giving up recreational activities, for example exercise, participation in clubs, gardening and traveling. Fatigue also compromised future plans. Some described that they did not make long term plans related to their goals in life; others mentioned that they did not make mid-term plans, for example vacation or attending social events because they were concerned that they would not be able to follow-through due to their exhaustion. Participants described that they lived day-by-day and often the plans they made for the day were compromised because of their fatigue. A participant described that her ongoing fatigue may influence her decision about the treatment plan of her liver cancer.

Well that comes down to the decisions that I make. I mean I could make the decision that I don't want you doing anything for me. Just leave me and let me go. Or I could have this risky surgery. So it’s hard to tell right now. I want; I look forward to the surgery that they were talking about; yesterday they found another area of concern. So it could possibly be two surgeries. I'm too tired to do that right now, you know what I mean? I’m just tired and I don’t, I don't know. I asked them about quality of life, on both aspects and he didn’t want to say anything until he gets more information back. Twenty nine percent of participants reported difficulties related to finances. Some mentioned that fatigue affected their financial situation because of lost income due to their impaired ability to work. Others described that they did not have the energy to organize or to pay 
their bills or that they spent significant amounts on nutritional supplements and alternative medications to treat their fatigue and their liver disease.

Although fatigue affected the lives of each participant in some way, there were some in the sample who emphasized that one can have a high quality of life despite having fatigue.

I would hate to think that in this survey that you're portraying that you know this is something that is insurmountable because it's not, I think that with just some thought and planning and good nutrition and some good health habits you know a lot can be done; it doesn't have to bring you down.

Communication about fatigue with health care providers. Participants discussed that they spoke about their fatigue to their primary care providers or to the gastroenterologist. The participants’ description revealed that family physicians or nurse practitioners had done a thorough investigation to try to find the causes of fatigue. In some cases, it led to the diagnosis of the HCV infection; in others, with already known HCV infection, they could not find any alternative cause. Based on the participants’ descriptions, advice on specific fatigue management strategies was not offered, but the participants appreciated they were listened to and their problem was taken seriously by their primary health care providers.

I think he's pretty much, it’s so much he says stuff about it, he thinks, the thing like you he’s trying to learn about it at the same time. So he's questioning me and I think he’s trying to learn for himself too. So he knows so much about it but at the same time he’s asking questions so he’s trying to learn and get basically a handle on how you know, cause I guess he's trying to figure out too what part is your disease playing on it and how much of it is you know, me driving back and forth. And then my work day, my 
medications that I've gone through. So I think it's not so much what he tells me it's, I think he kind of works through it with me.

"I think that she’s done her... I mean I don't know. I think she's done what she can do...which is to send me to people.”

"My family doctor ran every imaginable test in the world to find out what the fatigue was about and didn’t come up with anything other than it’s probably your hepatitis C.”

I've mentioned it to my family physician probably, I don’t even know how many times, every time I have my yearly physical, every time I go see him about how tired I am but nothing is really done about that. I haven't really been given any advice as to what I could change to help me feel better so I haven't really changed much. I just kind of work around, around the fatigue.

Based on their communication with the gastroenterologists, participants' understanding was that the specialists had clear opinions about the relationship between the liver disease and fatigue. In some cases, they reported that the gastroenterologist told them that the cause of the fatigue was the infection.

Even when I was there to see the doctor I explained to him you know that I've been feeling really tired, really tired, you know like not tired, sleepy tired but fatigued. My body starting to, you know, shut down insofar as I can’t do much after you know. And he could only say so much to me because he knows as a liver doctor that, why that's happening is the hep C and that's what he said. He said "It's the hep C that's doing this." Others heard the opposite opinion from their treating physicians. Hearing from the specialist that fatigue may have had causes other than the infection led some patients to retrospectively reevaluate the impact of the infection and fatigue on their lives: 
I have mentioned it to him but I know that he said to me, he said physical fatigue is not always a sign of the hepatitis C. I thought that was interesting. It seemed to confuse me at the time 'cause I always thought that my physical fatigue was very much a part of the hep C. But he said that it could be a sign of, being depressed and I thought, after I thought you know “okay” that quite, 'cause he says he’s had patients that have hep C who are not tired... and that's when I started to really associate lifestyle with ... Yeah it's true you know. I could do a lot of things even with the hep C. It's just, it, what I've tried to do has not worked out not the fact that I have the hep C. You know so I do agree with him on that you know. But again it’s so intertwined I think you know.

Another patient reported difficulty generating new causal attributions to the fatigue after hearing from the specialist that the infection was not the cause of fatigue:

I went to see him because I hadn’t seen him for eight years and he ran all kinds of blood work and everything and said; he said, and he's the expert: the fatigue has nothing to do with the hepatitis C.” So if that's true than I don't know what this is about.

This patient had a theory about the association between the infection and fatigue that was not challenged by the physician's statement because no alternative explanations were available:

The reason that I did attribute it to hep C was that when I was diagnosed, it was the fatigue that took me to the doctors... I have to let it go though, because both doctors have told me it’s not. But I don't know, I have no idea...Like, I keep associating the fatigue with hepatitis C but even the specialist said it’s not.

\section{Reasons for not communicating about fatigue with the health care providers.}

Those who do not discuss their fatigue with their physicians described their reasons for not communicating on this topic. One explanation that patients gave was that they did not bring up 
problems on their own during the medical encounters. Patients described that they did not bring up issues because they found the appointments to be too short to have time for both their and the doctors' agenda, so they let their doctors to determine the topics of the conversation:

"Same thing, he’s busy too. You go in there, you see him for 10 minutes and I let him direct the interview.”

Others explained that they generally did not share problems unless they were asked specifically:

"It tends not to come up in a general conversation and I tend, unless I'm asked something directly, I don’t say anything."

The relationship between the participants and their family doctors also contributed to the lack of communication about fatigue.

“I haven't had this doctor very long. Maybe five years and I'm, I don't want her to know. ... I don’t want her to know my fatigue. I don’t want her to know that.” Another participant discussed:

He didn’t really give me an opportunity to talk to him about any of this...Wasn’t given any opportunity. No matter what's gone on between him and I in the last few years, yeah he's just discounted me. Now I think he has a prejudice around how I most likely got hepatitis and he’s just dismissed me ever since...And I've had him for 20 years and for the first 15 we got along really well, I really trusted him, I thought he was a great doctor. And then in the last five we have not gotten along at all."

The final set of reasons stems from the participants' beliefs about fatigue and the treatability of fatigue. In particular, patients described that they knew the cause of their fatigue, so mentioning it to the physician would not have yielded any benefit, especially because they 
believed that fatigue could not be treated. Some also thought that fatigue was not an appropriate reason for going to the physician because it was not a serious or a specific complaint.

I tell the doctor nothing unless it's specific why I'm there. If I go and see a doctor there's a reason. I got a pain, like I told you this thing right there too, lately. I've had it before, he knows what it is and I want to, I want to know exactly what it is and how to take care of it... There's no sense in going through with that with the doctor unless it’s a very specific... There's no sense. I basically already know where it's coming from... when we get to these questions, basically it will help in a way but I already know the answers to them. There is no real answer to the way I feel. Like I have hepatitis C, it's the virus that causes this.

Another participant commented as follows:

I guess because I say to myself, I say “Okay I know I have hep C, I know it’s my problem, I know what's going on in my body and what's happening is because of it and there's nothing she can do so why bother talking to her about it?

Someone else said:

I don't know what they could do. They might be able to come up with some solution. But as I say I just don’t know, I don’t know what if any treatment there is for fatigue except for just living with it.

\section{Mixed method results: Fatigue Attributions}

This section describes the fatigue attributions provided by the whole sample in response to the BIPQ “causes” item. The BIPQ “causes” item asks participants to identify and rank-order the three most important causes of their fatigue. 
Participants provided a total of 254 fatigue attributions on the BIPQ. The three most frequent attributions in the whole sample were in decreasing frequency (a) HCV infection, (b) sleep (including inadequate sleep quantity/quality; unhealthy sleep hygiene and insomnia), and (c) other medical conditions and work (including overwork and work schedule). There was a difference in the frequency of attributions in the in the less fatigued (FSS $<4$ ) and the high fatigued (FSS $\geq 4$ ) groups, wherein the three most frequent attributions in the former group were: (a) sleep, (b) other medical conditions, and (c) physical activity (including exercise) whereas in the high fatigue group were: (a) HCV infection, (b) sleep, and (c) work. There was a significant association between the level of fatigue (low versus high) and whether participants attributed their fatigue to the HCV infection $\left(\chi^{2}=8.23, p<.05\right)$. This reflects the fact that, according to the odds ratio, those in the moderate-high fatigue group were three times more likely to attribute their fatigue to the liver infection than participants in the low fatigue group.

The "most important cause of fatigue" (i.e. the first attributions on the BIPQ list of causes) were also compared between the normal fatigue and the high fatigue groups. HCV infection was the top fatigue attribution in the severely fatigued group. It was ranked as the most important cause of fatigue by $37 \%$ of the participants in this group. There was a significant association between the level of fatigue (low versus high) and whether participants identified the infection as the most important cause of their fatigue $\left(\chi^{2}=6.25, p=.01\right)$. According to the odds ratio, participants with severe fatigue were four times more likely to believe that the most important cause of their fatigue was the HCV infection than participants with lower fatigue (Table16).

Insert Table 16 here 


\section{Discussion}

The purpose of the study was twofold: (a) to explore and understand fatigue as it manifests in participants' lives, and (b) to consider possible factors that contribute to adverse fatigue outcomes in patients with chronic HCV infection. The quantitative analyses confirmed that fatigue was an issue in the patient group, i.e., almost $60 \%$ of the sample suffered from severe fatigue-related functional impairment. This is consistent with other studies suggesting that fatigue is among the most frequent patient-reported concerns in relation to disease impact and treatment effects in chronic HCV infection (Kleinman, et al., 2012). The mean score of the present sample on the Fatigue Severity Scale (FSS) was higher than the mean values in healthy controls and also higher than the mean pre- treatment score of a large group of HCV infected patients in an international multi-center clinical trial (3.8) (Ettinger et al., 1998; Herlofson \& Larsen, 2002; Kleinman, et al., 2000; Krupp, LaRocca, Muir-Nash, \& Steinberg, 1989b; Valko, Bassetti, Bloch, Held, \& Baumann, 2008). The mean FSS scores in this sample were commensurate with the FSS scores in medical conditions that are known to be associated with high fatigue, for example sleep disorders (4.3) and multiple sclerosis (4.6-4.8) (Aguillard et al., 1998; Krupp, et al., 1989b; Sanchez-Ortuno, Edinger, \& Wyatt, 2011). Based on a review on patient reported outcomes in HCV infection, it has been recommended that the FSS be included in HCV clinical trials as an important patient reported outcome measure (Kleinman, et al., 2012). The patients' descriptions confirmed that the FSS items tap into issues that are relevant for individuals with HCV infection: physical activity as a trigger of fatigue; and functional impairment in life domains specified in the FSS. The causal attributions emerging from the interviews and from the "causes” item the BIPQ were also consistent with the FSS that refers to fatigue as one of the top three perceived symptoms of the illness. On the other hand, 
approximately half of the qualitative sample discussed two areas of functional impairment, namely "active recreation" and "future plans" that are not specifically captured by the FSS. In summary, the study results support previous findings that suggest fatigue may be a clinically significant issue among patients with chronic HCV infection treated in tertiary urban hepatitis centers. The FSS appears to be a sound tool to assess the impact of fatigue, although it does not specifically address two areas that fatigue has an impact on: functional impairment and future plans.

Two hypotheses were put forward regarding the factors that contribute to the impact of fatigue. The primary hypothesis was that insomnia, depression, anxiety symptoms, as well as fatigue specific cognitions and behaviours would predict fatigue above and beyond presence of liver disease and medical comorbidities. The results partially supported this hypothesis. Whereas there was no statistically significant contribution for liver disease, the strongest predictors of fatigue were two domains of "fatigue perception": concern about fatigue and control over fatigue. The association between illness perceptions and fatigue, in some ways, is not surprising, given that this relationship has been shown in some medical conditions. For example "illness perceptions” (IPQ-R) accounted for approximately one third of the variance in physical fatigue and in mental fatigue scores in two studies in multiple sclerosis, whereas disease measures did not significantly predict fatigue (Jopson \& Moss-Morris, 2003; Moss-Morris, et al., 2002). Similar to the findings of the current study, the "control" items had a unique, significant value in predicting physical and mental fatigue. The role of illness perceptions (including control and concern) in predicting fatigue has been further supported in prospective studies (Broadbent, et al., 2006; Moss-Morris, Spence, \& Hou, 2011). 
The above mentioned findings underscore the importance of illness perceptions in predicting fatigue. However, they are different in one important respect: the studies cited above assessed patients' perceptions of their respective illnesses and their association with fatigue; whereas the current study examined the potential role of fatigue perceptions specifically in predicting fatigue-related outcomes. The key role of fatigue-specific cognitions in the psychopathology of fatigue has been conceptually described in the cognitive behavioural fatigue model, and is supported by treatment studies in chronic fatigue syndrome and cancer fatigue (Knoop, Prins, Moss-Morris, \& Bleijenberg, 2010)

In addition to illness perception, depression symptoms were a lesser but nonetheless significant predictors of fatigue. The strength of association between the depression symptoms and the FSS in the current study (.5) was similar to what was found in a study at a psychiatric clinic treating patients with chronic hepatitis C infection in Canada (.4) (Sockalingam, et al., 2011). The predictive value of depression symptoms (before the inclusion of the cognitive variables) in the current study was comparable to the results of a previous study at a tertiary liver clinic (Dwight, et al., 2000). Beyond these similarities, there were some important differences between the current and the previous two studies on depression. Whereas the previous studies examined differences in mean scores between "depressed" and "non-depressed" groups or included only the severity of the liver disease and the depression scores in the regression model, the current study considered other, theoretically important factors that may account for fatigue in hepatitis C infection, including fatigue-specific cognitions. These cognitions had a larger, unique contribution to fatigue outcomes than did the depression, which underscores the importance of studying these cognitions (above and beyond depression) with regards to fatigue in this population. 
Unlike depression and fatigue cognitions, there was no statistically significant effect for anxiety and fibrosis severity on fatigue. The contribution of anxiety to fatigue has not been studied in chronic HCV infection but it has been shown in some other medical conditions, for example in cancer (Brown \& Kroenke, 2009). The challenge with anxiety assessment in fatigue research has been the lack of consistency in the constructs measured. The specific issue with some popular anxiety assessment tools (e.g. State-Trait Anxiety Inventory) in the context of medical conditions is that they assess both the symptoms of depression and anxiety (Antony, et al., 1998; Bieling, Antony, \& Swinson, 1998; Campbell-Sills \& Brown, 2011; Spielberger, Gorsuch, Lushhene, Vagg, \& Jacobs, 1983). The advantage of using the DASS in the current study was that this measure distinguishes between the specific symptoms of anxiety and depression (Antony, et al., 1998). Future research will need to use specific anxiety measures to determine the contribution of well-defined anxiety constructs to fatigue.

In addition to the non-significant effect of anxiety symptoms, there was also no statistically significant effect for the degree of liver fibrosis. The non-significant result for liver disease severity is consistent with the limited number of previous studies in hepatitis $\mathrm{C}$ infection (Dwight, et al., 2000; Goh, et al., 1999; Poynard, et al., 2002; Wessely \& Pariante, 2002). In general, measures of the severity/duration/stage/intensity of medical conditions have not been significantly related to fatigue (DeLuca, 2005). This observation can be explained on both methodological and theoretical grounds. In terms of methodology, if the outcome measure is assessed with the same method as some predictors are measured, the strong correlation between these predictors and the outcome may be, in part attributable to shared method variance. In the current study the fatigue measure and predictors were assessed with Likert type questionnaires. The liver fibrosis and the comorbid conditions were the only predictors that did not share this 
assessment method. Therefore, the regression results, in part could be attributable to the choice of the assessment methods. In addition to the shared method variance, a second possible explanation relates to the fact that the measure of liver fibrosis has a limited range. Fibrosis is the "gold standard" measure of liver disease severity and is used clinically to inform decisions on the initiation of antiviral treatment. Controlling for the severity of liver disease was a theoretically reasonable approach. However, because the range of fibrosis stages (zero to five) was limited, this may have restricted the predictive value of this measure in the regression model.

From a theoretical perspective, inflammatory markers may be better candidates for predicting fatigue than disease severity measures. Pro-inflammatory cytokines induce fatigue in acute and chronic infections or when they are administered to treat infections, as for example interferon treatment does in hepatitis C infection (Reichard et al., 1998; Zeuzem, et al., 2000). The challenge with respect to causal conclusions about disease-specific changes in cytokine levels and fatigue in chronic medical conditions is that common concomitant medical conditions with fatigue symptoms, in particular sleep disorders, depression and obesity may also alter cytokine secretion (Ciftci, Kokturk, Bukan, \& Bilgihan, 2004; Hotamisligil, 2006; Irwin \& Miller, 2007; Okun et al., 2011; Vgontzas et al., 2000). Given the high rate of clinically significant sleep complaints in the current sample, it would be difficult to disentangle the differential effect of liver infection and sleep disorders on potential changes in cytokine levels.

In summary, based on the results it is unclear whether liver disease specifically contributes to fatigue in chronic hepatitis $\mathrm{C}$ infection. Physiological processes probably contribute to the experience of fatigue but these physiological changes may reflect the combination of factors originating from the liver disease, co-morbidities (e.g. sleep disorders), medications, and behavioural/lifestyle factors. The theoretical implication of the primary results 
is that patients' perception of their fatigue may have a unique contribution to adverse fatigue outcomes above and beyond the liver disease and the symptoms of its comorbidities.

The secondary hypothesis predicted, and post hoc mediation analyses confirmed that fatigue perceptions may have a unique role in providing a link between medical/mental health conditions and fatigue-related functional impairment. The mediation results suggest that depression may influence patients' perceptions of their fatigue. This, in turn, may amplify the perceived intensity of fatigue and worsen fatigue outcomes. Indeed, previous studies have consistently shown that individuals with chronic medical conditions and comorbid depression report more physical symptoms and experience more significant illness-related functional impairment than patients without depression, independent of the disease severity (Rodin, Craven, \& Littlefield, 1991). This was also reflected in the previously described study on depression and fatigue in chronic HCV infection (Dwight, et al., 2000). Dwight et al (2000) did not show a difference between the mean fatigue scores of "depressed" and non-depressed patients. On the other hand, they noted that the "depressed” group reported more fatigue-related functional impairment than the non-depressed group even though there was not a significant difference in any objective measure of liver disease severity or liver enzyme levels between the two groups.

Although fatigue is the leading daytime symptom of insomnia, the relationship between insomnia and fatigue appears to be more complicated than a straightforward cause and effect. In the present study insomnia predicted fatigue but it exerted its effect through fatigue-specific cognitions. This finding supports contemporary, cognitive conceptualizations of insomnia. The cognitive model of insomnia posits that individuals' cognitions (i.e. their beliefs about sleep, monitoring physical sensations, attention bias to sleep “threats”, and misperceptions of sleep duration) increases arousal and fuels safety behaviours that maintain fatigue (Harvey, 2002). 
Indeed, individuals with insomnia appear to be excessively concerned about possible or actual daytime fatigue (Harris \& Carney, 2009). These individuals therefore may engage in avoidance behaviours in order to reduce their "fear of fatigue" and the probability of experiencing fatigue. Avoidance, in the face of non-harmful events and physical sensations, is non-adaptive, because it may increase the probability of a feared event to happen and may lead to actual functional impairment. For example, in the context of insomnia, concern (or fear) of fatigue may lead to avoidance behaviours (e.g. spending an extended period of time in bed) that leads to adverse consequences (e.g. decreased sleep continuity and disruption of the circadian rhythm), which amplify fatigue.

In summary, comorbid medical conditions appear to contribute to fatigue in chronic HCV infection, in part through cognitive pathways. In other words, not (only) the symptoms of comorbidities, but also specific fatigue cognitions in the context of these comorbidities are associated with the functional impact of fatigue. Fatigue cognitions, along with patients’ perceptions of fatigue and fatigue management behaviours were explored in the qualitative component of the study, described herein.

In the qualitative component of the study, the main cognitive themes were fatigue attributions, prognosis, control, cognitive avoidance, acceptance, and positive attitude. Most participants in the qualitative sample attributed their fatigue to the HCV infection. This was in line with the finding that in the full sample of 115 participants those with significant fatigue were four times more likely to say that the most important cause of their fatigue was the infection. The association between somatic fatigue attributions and intense fatigue is consistent with research in chronic fatigue syndrome, cancer and multiple sclerosis (Bol, Duits, Hupperts, Vlaeyen, \& Verhey, 2009; Servaes, Verhagen, \& Bleijenberg, 2002; Sharpe, 1997). Attributing fatigue to 
the infection was mentioned in relation to other themes by the participants in the present study, including the course of fatigue, the prognosis of fatigue, acceptance, and communication about fatigue. In terms of the course of fatigue, participants reported that their infection was chronic and they experienced chronic fatigue, and therefore reasoned the two must be related. Some participants described that they already had intense fatigue when they received the diagnosis of the infection and these participants made the causal connection between the fatigue and the infection soon after the diagnosis. These reports are similar to those described in a previous qualitative study on fatigue in HCV infection (Glacken, et al., 2001). Prospective, quantitative data also shows that baseline fatigue is the strongest predictor of persistent fatigue in cancer survivors (Servaes, Gielissen, Verhagen, \& Bleijenberg, 2007).

In line with a previous qualitative study, participants in the present study reported that they experienced negative emotions after they became aware that they were fatigued (Glacken, et al., 2003). They also associated the fatigue with illness progression. Previous studies in cancer, multiple sclerosis, chronic fatigue syndrome and fibromyalgia have consistently shown that being fatigued may become emotionally threatening and distressing. This is particularly the case if patients believe fatigue is a sign of disease progression/recurrence/flair-up (Lukkahatai \& Saligan, 2013). There is evidence to support that negative emotions can amplify unpleasant physical sensations because they increase self-focused attention and monitoring (Mor \& Winquist, 2002).

Most patients in the qualitative sample reported that they have limited/no control over their fatigue. These were the individuals whose primary fatigue management behaviours were fatigue-driven. The fatigue-driven behavioural pattern included a set of passive coping behaviours. These behaviours were passive in a sense that they were reactive to the experience of 
fatigue. These behaviours were also escape/avoidance behaviours because their goal was to reduce the intensity of the fatigue sensation. Avoidance is a life-saving coping behaviour in situations that are dangerous. On the other hand, persistent avoidance of non-harmful life events or benign physical sensations is non-adaptive because it prevents new learning, restricts the individual's behavioural repertoire and may render temporary, benign physical sensations of pain or fatigue chronic (Bol et al., 2010; Leeuw et al., 2007). Participants in the present study described pacing as an effective behavioural fatigue management strategy that some participants also associated with high sense of control. Nevertheless, participants using pacing as a fatigue management strategy also reported high fatigue-related impairment on the FSS. Emerging evidence has found that pacing has mixed support for patients in terms of improving fatigue/pain intensity (Karsdorp \& Vlaeyen, 2009; Murphy, Smith, \& Alexander, 2008; White et al., 2011). Cognitive avoidance also emerged as a separate theme in the interviews. Based on the interview data it cannot be determined whether cognitive avoidance was rooted in control beliefs or whether it was an adaptive or a non-adaptive coping strategy in the present sample. The qualitative data suggests that beliefs about the treatment control of fatigue were associated with communication about fatigue. Specifically, those who believed that there is no treatment for fatigue did not mention the problem of fatigue to their physicians. The low fatigue treatment control representations, in some cases were present in parallel to high HCV treatment control beliefs. In these cases, participants believed that they could not get medical help for their fatigue, and their fatigue would increase. At the same time they believed that a new antiviral medication would cure their infection and as a result, their fatigue would dissipate.

Acceptance of fatigue emerged as a cognitive coping strategy in qualitative sample. Acceptance of physical sensations is adaptive in chronic conditions that cannot be cured by 
routine medical interventions, for example in chronic pain conditions or in chronic fatigue syndrome (Brooks, Rimes, \& Chalder, 2011b; McCracken \& Vowles, 2006, 2008; McCracken, et al., 2004; Van Damme, Grombez, Van Houdenhove, Mariman, \& Michielsen, 2006). In the current qualitative sample, however, based on participants' reasoning, ascribing fatigue to the infection and accepting to have chronic fatigue as a "symptom" of the infection, prevented some patients from sharing their fatigue problem with their family physicians, who therefore did not even have the opportunity to intervene. In the event when fatigue is caused by well-treatable conditions, for example by sleep disorders, accepting chronic fatigue as an inevitable part of the infection is probably non-adaptive.

The above findings are in line with evidence from studies of other medical conditions, and imply that fatigue attributions, concern about fatigue, beliefs about the timeline, controllability/curability of the illness and fatigue, acceptance and fatigue management behaviours are important factors to consider in association with fatigue in HCV infection. Further, there appears to be a relationship between the beliefs about fatigue and about the HCV infection, and these appear to be associated with behavioural adaptation to fatigue. These results support the cognitive behavioural and illness perception models of chronic fatigue (Moss-Morris, 2005; van Kessel \& Moss-Morris, 2006). The association between insomnia, depression symptoms and fatigue traditionally has been interpreted in the framework of the two-stage fatigue model, according to which "secondary" medical conditions (i.e. insomnia, depression) maintain "secondary" fatigue in the medically ill (DeLuca, 2005). However, the current mediation results suggest that patients may endorse fatigue cognitions in the context of insomnia, depression or other comorbidities and these cognitions may specifically contribute to fatigue outcomes. A possible way to integrate the above findings is to consider the possibility that 
multiple medical conditions are comorbidities (instead of “primary” and “secondary” disorders) that may equally contribute to fatigue outcomes. Further, the way patients make sense and adapt to their (comorbid) illnesses (e.g. liver disease, insomnia, and depression) may be linked to their beliefs and adaptation to fatigue, and the these processes shape the experience and outcomes of fatigue. An integrated theoretical model depicting these relationships is presented in Appendix E.

As described above, the primary perceived cause of fatigue was the infection in the high fatigue group, according to both the quantitative and qualitative results. Sleep problems were the second most frequent perceived causes of fatigue. Additionally, patients in the interviews discussed sleep problems as the cause or the trigger of their fatigue. The sleep disorder screening results confirmed the final hypothesis of the study with respect to the high rate of insomnia and obstructive sleep apnea symptoms. The screening showed that more than one third of the sample reported moderate and severe symptoms of clinical insomnia. The rate of clinical insomnia in the sample was indeed higher than the prevalence rate for insomnia (i.e., $10 \%$ ) (Morin, LeBlanc, Daley, Gregoire, \& Merette, 2006; Ohayon, 2002). Based on cut-off scores on the STOP-BANG questionnaire, $70 \%$ of the sample had possible obstructive sleep apnea and almost 30\% had increased risk for moderate or severe obstructive sleep apnea. The distribution of the STOPBANG scores in the current sample was similar to the distribution in a sample of 750 patients waiting for surgery at the University Health Network and Mount Sinai Hospital (Chung, et al., 2012). In the above pre-surgical sample $68.4 \%$ had sleep apnea based on their polysomnography results. Approximately 50\% of those with clinically significant sleep apnea symptoms also reported clinical insomnia. This reflects the proportion of individuals with comorbid obstructive sleep apnea and insomnia, increasingly referred to as "sleep apnea plus” in the literature 
(Luyster, Buysse, \& Strollo, 2010; Vozoris, 2012). In summary, the sleep disorder screening results suggest that moderate/severe insomnia and obstructive sleep apnea may have been clinical issues in the sample. These disorders require clinical attention not only because they are associated with fatigue and excessive daytime sleepiness, but also because they have serious public health and economic consequences (Kessler et al., 2011; Leger, Bayon, Laaban, \& Philip, 2012).

\section{Clinical Implications}

Taken together, the findings that have possible clinical implications are: (a) fatigue was a clinically significant issue for patients in this study; (b) patients' beliefs about their fatigue were significant predictors of fatigue-related functional impairment; (c) approximately $50 \%$ of patients reported clinically significant symptoms of insomnia, obstructive sleep apnea or a combination of both; (e) patients with insomnia or with the combination of insomnia and clinically significant sleep apnea symptoms reported severe fatigue outcomes; and (f) some patients were reluctant to communicate about their fatigue with their health-care providers. Based on these findings it may be worthwhile to test the following interventions in future research: training healthcare providers and patients how to communicate effectively about fatigue; fatigue/sleep disorder screening and specific fatigue treatments. Ideas for these possible future interventions are discussed below.

With respect to communication, consistent evidence supports that patients' illness perceptions and their perceptions about the relationship with physicians determine what information they share, how they present the information, whether they consent to treatment, their treatment adherence, and treatment outcomes (Bensing \& Verhaak, 2004). Some patients in the qualitative sample stated that they would not mention their fatigue to their physicians unless they are specifically asked about that issue. Participants also noted that they perceive the medical 
visits as being too short to have time for both their and the physicians agenda, so they let their doctors determine the topics discussed. Giving an opportunity for patients to bring up items from their own agenda has been associated with improved health outcomes in hypertension, diabetes, peptic ulcer, and breast cancer (Crow et al., 1999). Asking patients directly whether they experience fatigue that interferes with their daily activities may open a door for patients to share their concern about fatigue. It also could transmit the message to patients that fatigue is a legitimate problem to discuss. This may be important for patients who believed that fatigue was not a legitimate reason to book a medical appointment with the family doctor as was reported by a number of individuals in the study.

The placebo effect demonstrates that treatment expectations can be as powerful as specific interventions on treatment outcomes (Price, Finniss, \& Benedetti, 2008). Interview participants in the present study explained that they did not discuss their fatigue with their physicians because they believed there is no treatment for fatigue. Negative expectations about treatment and negative aspects of clinical visits can cause adverse disease or treatment outcomes, sometimes also referred to as "nocebo effect” and "nocebo response” (Colloca \& Miller, 2011). Not only patients', but also the physicians' expectations about treatment outcomes may influence the intensity of symptoms that the patients experience (Galer, Schwartz, \& Turner, 1997). Fatigue, in this respect, may flourish in a "lose-lose" situation, created by low treatment expectations on both the patients' and the physicians’ part. In general terms, patients who discussed their fatigue with their physicians reported that the family doctors, despite their good intentions, could not provide effective help or useful advice on how to cope with fatigue. It is to be determined, if patients benefited from simple, individually tailored fatigue management 
advice given by family doctors or from receiving an educational booklet specifically on coping with fatigue in medical illnesses.

As the qualitative results in this study indicate, patients may attribute their fatigue to the infection because they are not able to find other, plausible explanations for fatigue. Therefore, it may not be sufficient to simply tell patients that the HCV infection causes/ does not cause their fatigue. Discussing fatigue attributions openly and generating ideas with patients collaboratively about possible, multiple contributors to fatigue may help patients to consider alternative causal explanations (Harris \& Carney, 2012). This might allow patients to engage in alternative fatigue management strategies. There is evidence to support the notion that short interventions following this approach have multiple measurable benefits on disease outcomes (Broadbent, Ellis, Thomas, Gamble, \& Petrie, 2009).

Screening for fatigue and sleep disorders might yield clinical benefits for patients. A simple, one page screening tool could be completed by the patients in 5 minutes while they are waiting for their appointments. The physician could obtain valuable information in a single glance that could inform clinical decision making about potential referrals. An example for a one page screening tool is included in Appendix D. An example for an algorithm to guide decision making accompanies the screening tool (Appendix D). Particularly germane areas to assess may include: fatigue, concern about fatigue, sleep disturbance severity, and possible sleep-disordered breathing. Patients who are concerned about their fatigue may be more likely to experience fatigue-related functional impairment. These patients may benefit from further assessment and potentially from a fatigue-specific treatment discussed below. A question on global dissatisfaction about sleep is the second item of the screening tool. This item was included because a question about global dissatisfaction with sleep quality or quantity was the best item to 
detect insomnia syndrome and specifically, daytime symptoms of insomnia in an epidemiological sample of close to 9000 individuals in the United States (Ohayon, Riemann, Morin, \& Reynolds, 2012). Patients scoring highly on this screening question would benefit from a sleep assessment. Finally, the STOP-BANG questionnaire is suggested for screening for obstructive sleep apnea. Scores of 5 and above indicate that the patient is at high risk for moderate or severe sleep apnea (Chung, et al., 2012). The STOP-BANG scores should be evaluated in combination with the assessment of comorbidities that may be caused or complicated by untreated sleep apnea, including cardiovascular disorders, obesity, diabetes, depression or dementia. The suggestions above are based on the findings of the study as well as the literature to date; future studies could investigate the best way to approach fatigue assessment in this group, as well as in other patient groups.

Lastly, in addition to more effective communication about fatigue and engaging in screening for sleep and fatigue issues, patients need effective specific fatigue interventions. Graded exercise therapy and cognitive behavioural therapy have been shown to reduce fatigue in patients with chronic fatigue syndrome, cancer and multiple sclerosis in randomized clinical trials (Gielissen, Verhagen, Witjes, \& Bleijenberg, 2006; Goedendorp, Gielissen, Peters, Verhagen, \& Bleijenberg, 2011; Jacobsen, Donovan, Vadaparampil, \& Small, 2007; Montgomery et al., 2009; R. Moss-Morris et al., 2012; van Kessel et al., 2008; van Weert et al., 2010; White, et al., 2011). Brief interventions targeting patients' illness perceptions can be offered as stand-alone interventions to improve disease outcomes (Broadbent, et al., 2009).

\section{Limitations}

The results provide helpful insight into the nature of fatigue in these patients and point to possible treatment/assessment implications; nonetheless, the design, sample characteristics and 
data collection methods of the study may have implications for the interpretation of the results. The issue of shared method variance was discussed previously (i.e., the fatigue-cognition questionnaires and symptom questionnaires are paper-and-pencil self-report measures, just like the outcome measure). In essence, the shared method of data collection could be a methodological explanation for the observed relationship between the predictors and the outcomes. As has been alluded to above, assessing the constructs with multiple methods in future studies could address whether shared method variance plays any role in the results.

The regression and the mediation analyses imply a directional relationship between variables. It is important to emphasize that the relationship between variables tested in this study are associations, rather than causal relationships. Sound conceptual models and theoretical arguments do convincingly support the directional changes of variables but these relationships cannot be confirmed methodologically in a study with a cross sectional design. In general terms, from a methodological perspective, an experimental design or a longitudinal quasi experimental design are appropriate ways to investigate cause and effect relationships. Such designs were beyond the scope of the current study. Notwithstanding the methodological considerations, it is important to point out that the relationships between the variables depicted in the theoretical model are multi-directional (Figure 11). The best way to interpret the results is that the statistical models singled out and tested only one direction of multiple relations between selected variables. Although the collinearity statistics indicated that the association between predictors was appropriate for regression, the strong correlation between the predictors may have increased the possibility of Type 2 errors. Also, setting the alpha levels at .05 for the mediation analyses may have increased the possibility of Type 1 errors. 
A particular challenge in designing the research was the paucity of established questionnaires assessing fatigue-specific cognitions. The BIPQ and the CPAQ was adapted in the study based on two previously published projects (Brooks, et al., 2011b; Knoop, et al., 2012). The DISRS was used in a homogeneous medically ill population for the first time in the present project. All the questionnaires had excellent psychometric properties in the current study. The construct validity of the DISRS was supported by the fact that the mean score was almost identical to the mean score determined in the original validation study, and the highest scores were obtained by patients with comorbid insomnia. Nevertheless, the construct validation of these questionnaires in future research is warranted.

Fatigue management behaviours can be instrumental determinants of fatigue outcomes. The behavioural adaptation to fatigue was explored in the qualitative component of the project. On the other hand, fatigue-related behavioural data in the quantitative component was only collected using self-report on the "activity engagement" subscale of the CFAQ. Future research on fatigue perception could use multiple activity measures in order to test the relationship between fatigue perception, fatigue management behaviours and fatigue outcomes.

The data collection at a specialized liver clinic has multiple implications on the actual data generated in the study and on the generalizability of the results. First, when patients complete questionnaires at a medical clinic, they may over-report symptoms due to demand characteristics of the social situation. In this study specifically, participants provided data after their medical appointments and the outcome of these appointments may have influenced participants' perceptions of their well-being. Also, the information provided during the informed consent process (i.e. the researchers wanted to understand the issue fatigue) may have led to a selection bias and may have prompted patients to over-report their difficulties with fatigue. In 
order to reduce the validity threat of a potential selection bias, every patient visiting the clinic during the data collection phase was assessed for interest in participation. Patients were also told that the participation of everyone, including those who were not fatigued was desirable. The response rate was high (above 80\%) which decreases the probability of a significant selection bias. A second issue related to the characteristics of the data collection site concerns the generalizability of the results. Ideally, the study would have involved multiple sites of data collection. The specific results of the current study may be the most relevant to treatmentseeking, non-drug user patients, attending North American tertiary liver clinics. However, the general "take -home" messages of the study concerning the importance of fatigue and illness perceptions and untreated sleep disorders in fatigue outcomes may be generally relevant and worth exploring in different settings where patients with chronic HCV infection are treated.

As with the quantitative data, the qualitative data is also a product of interaction between the participants and the research context in a particular moment of time. In this component of the study there probably was a selection bias, since only those willing to discuss their fatigue volunteered to participate in the interviews. Also, patients staying at home are more likely to have time to participate in the qualitative component. It is also notable that there were more women than men in the qualitative sample, whereas more men than women reported severe fatigue outcomes on the FSS in the full sample.

The interviews were conducted by one researcher, which may have influenced the qualitative results. To reduce this bias, the interviewer (DZ) followed a written, semi-structured interview schedule that was approved by two supervisors before the data collection was commenced. Using a semi-structured interview schedule and collecting data until saturation increased the probability that crucially important information relating specifically to the research 
questions were not missed. The downside of the semi-structured interview format was that the standard questions limited and influenced the information that the participants provided. The author's orientation in behavioural medicine, behavioural sleep medicine and in cognitive behavioural therapy made a clear mark on the research questions, the research objectives and the research design. It is important to state that the results and their present interpretation do not tell "the whole truth” about fatigue in chronic HCV infection. Rather, they tell a story about actual clinical issues that could have clinical relevance for clinicians and patients and may inspire future research.

\section{Future Directions}

The study provided preliminary evidence on the significance of untreated sleep disorders and of illness/fatigue cognitions in association with adverse fatigue outcomes. These results indicate that factors beyond the pathophysiology liver disease are important determinants of fatigue impact in chronic HCV infection. Further studies should test the predictive values of variables identified in this study, specifically sleep disorders, fatigue perception, acceptance, control and fatigue management behaviours on fatigue outcomes using multiple, precise measurement tools (e.g. polysomnography, questionnaires, actigraphy, ecological momentary measures of fatigue, activity log). Prospective studies should test whether fatigue and other relevant variables (comorbidities, activity level, illness and fatigue perception) predict chronic fatigue after patients are diagnosed with the infection. If the predictive values of these variables are established, testing the efficacy of interventions targeting specifically the predictors of chronic fatigue should ensue. These interventions may involve screening and treatment of sleep disorders and short interventions targeting non-adaptive illness/fatigue cognitions and behavioural coping strategies implemented soon after the diagnosis to prevent/treat chronic 
fatigue. The current study indicates that sleep apnea may be common at clinics with similar patient characteristics as the present sample. The distribution of STOP-BANG scores was very similar in this sample and in a large validation study of the STOP-BANG questionnaire but validating the questionnaire specifically in patients with chronic HCV infection would be worthwhile given the significant outcomes of untreated sleep apnea.

In conclusion, this study was the first to integrate qualitative and quantitative data on fatigue-outcomes, fatigue cognitions, and behaviours in chronic hepatitis C infection. The study confirmed that fatigue-related functional impairment is a problem that requires clinical attention in this population. The study provided preliminary evidence that modifiable factors, such as sleep disorders, fatigue cognitions, and coping behaviours are associated with the impact of fatigue in chronic hepatitis $C$ infection. Integrating these findings with existing evidence-based treatments for insomnia, sleep apnea, and fatigue could improve patient care and disease outcomes. 
Table1.

The Subtypes and Assessment of Physiological and Psychological Fatigue

\begin{tabular}{lll}
\hline Categories & Subtypes & Assessment \\
\hline Physiological & Peripheral & Force transducer, sEMG \\
& Central & \\
& & Magnetic stimulation, \\
& & EEG, performance in \\
\hline Psychological (Central, & Motivational & neuropsychological tests, \\
Subjective, Cognitive) & Cognitive & functional neuroimaging \\
& Physical & Questionnaire, clinical \\
& Emotional & interview, qualitative \\
& & research methods \\
\hline
\end{tabular}


Table 2.

Normal and Pathological Fatigue

Normal fatigue

Pathological fatigue

normal, adaptive function

temporary

persistent

relieved by rest

not relieved by rest

can be pleasant

distressing

does not impact on normal functioning

interferes with normal functioning

no impact on quality of life

negative impact on quality of life 
Table 3.

Factors Contributing to Fatigue in General Medical Conditions

\begin{tabular}{|c|c|c|c|c|c|c|c|c|c|}
\hline $\begin{array}{l}\text { Comorbid } \\
\text { Conditions }\end{array}$ & Cancer & HIV & $\begin{array}{l}\text { Chronic } \\
\text { kidney } \\
\text { disease }\end{array}$ & SLE & RA & pSS & $\begin{array}{c}\text { Hepatitis } \\
\text { C }\end{array}$ & CFS & COPD \\
\hline Depression & + & + & + & + & + & + & + & + & + \\
\hline Anxiety & + & + & $?$ & $?$ & ? & + & $?$ & ? & $?$ \\
\hline Sleep & + & $?$ & + & + & + & + & $+*$ & $?$ & $?$ \\
\hline Pain & + & ? & + & + & + & + & ? & ? & ? \\
\hline
\end{tabular}

Note. HIV= HIV infection, SLE = systemic lupus erythematosus , RA = rheumatoid arthritis, pSS = primary Sjögren syndrome, $\mathrm{CFS}=$ Chronic fatigue syndrome, $\mathrm{COPD}=$ chronic obstructive pulmonary disease

+ evidence suggesting an association

? not studied

*one study on sleep and fatigue during antiviral treatment 
Table 4.

Participant Characteristics $(N=115)$

\begin{tabular}{|c|c|c|c|c|c|}
\hline & Mean & SD & Range & $\mathrm{N}$ & $\%$ \\
\hline Age (years) & 56 & 10 & $23-82$ & & \\
\hline \multicolumn{6}{|l|}{ Gender } \\
\hline Male & & & & 72 & 63.5 \\
\hline Female & & & & 42 & 36.5 \\
\hline \multicolumn{6}{|l|}{ Cultural identity } \\
\hline Canadian & & & & 79 & 68.7 \\
\hline European & & & & 22 & 19.1 \\
\hline East-Asian & & & & 4 & 3.5 \\
\hline South -Asian & & & & 3 & 2.6 \\
\hline African & & & & 3 & 2.6 \\
\hline Caribbean & & & & 3 & 2.6 \\
\hline \multicolumn{6}{|l|}{ Relationship status } \\
\hline Married/ Common law & & & & 65 & 57 \\
\hline In a relationship & & & & 9 & 7.9 \\
\hline Not in a relationship & & & & 40 & 35.1 \\
\hline Diagnosis of HCV (years) & 13 & 9 & $0-35$ & & \\
\hline \multicolumn{6}{|l|}{ Fibrosis stage } \\
\hline F0 & & & & 11 & 9.8 \\
\hline $\mathrm{F} 1$ & & & & 20 & 17.9 \\
\hline $\mathrm{F} 2$ & & & & 10 & 8.9 \\
\hline F3 & & & & 21 & 18.8 \\
\hline $\mathrm{F} 4$ & & & & 50 & 44.6 \\
\hline \multicolumn{6}{|l|}{ Antiviral treatment } \\
\hline Received & & & & 58 & 50.40 \\
\hline Has not received & & & & 57 & 49.60 \\
\hline Number of comorbid medical conditions & 3 & 2 & $0-9$ & & \\
\hline Regular Exercise/ physical work & & & & 69 & 60.00 \\
\hline
\end{tabular}


Table 5.

Participant Characteristics- Qualitative Sample $(N=14)$

\begin{tabular}{|c|c|c|c|c|c|}
\hline & Mean & $\mathrm{SD}$ & Range & $\mathrm{N}$ & $\%$ \\
\hline Age (years) & 54 & 8 & $36-62$ & & \\
\hline \multicolumn{6}{|l|}{ Gender } \\
\hline Male & & & & 5 & 36 \\
\hline Female & & & & 9 & 64 \\
\hline \multicolumn{6}{|l|}{ Cultural Identity } \\
\hline Canadian & & & & 12 & 86 \\
\hline European & & & & 2 & 2 \\
\hline \multicolumn{6}{|l|}{ Relationship Status } \\
\hline Married/ Common law & & & & 7 & 50 \\
\hline Not in a relationship & & & & 7 & 50 \\
\hline Diagnosis of HCV (years) & 14 & 9 & $0-26$ & & \\
\hline \multicolumn{6}{|l|}{ Fibrosis Stage } \\
\hline F0 & & & & 2 & 14 \\
\hline $\mathrm{F} 1$ & & & & 1 & 7 \\
\hline F3 & & & & 2 & 14 \\
\hline $\mathrm{F} 4$ & & & & 9 & 64 \\
\hline \multicolumn{6}{|l|}{ Antiviral treatment } \\
\hline Received & & & & 9 & 64 \\
\hline Has not received & & & & 5 & 36 \\
\hline FSS & 5.35 & 0.87 & $4.00-6.67$ & & \\
\hline
\end{tabular}


Table 6.

Descriptive Characteristics of Study Variables

\begin{tabular}{|c|c|c|c|c|c|c|c|}
\hline Variables & Mean & $\begin{array}{c}\text { 95\% bias } \\
\text { corrected CI }\end{array}$ & Median & SD & Minimum & Maximum & $\begin{array}{c}\text { Interquartile } \\
\text { range }\end{array}$ \\
\hline FSS & 4.25 & $3.93-4.52$ & 4.67 & 1.65 & 1.00 & 7.00 & 2.92 \\
\hline ESS & 9.29 & $8.34-10.24$ & 9.00 & 4.99 & 0.00 & 23.00 & 8.00 \\
\hline ISI & 11.69 & $10.20-13.17$ & 11.00 & 7.72 & 0.00 & 28.00 & 11.00 \\
\hline DASS Anxiety & 3.92 & $3.17-4.67$ & 3.00 & 3.72 & 0.00 & 13.00 & 7.00 \\
\hline DISRS & 41.97 & 39.22-44.44 & 41.00 & 13.77 & 20.00 & 80.00 & 22.00 \\
\hline CFAQ activities engagement & 41.20 & 38.83-43.79 & 41.00 & 12.94 & 3.00 & 65.00 & 21.00 \\
\hline $\begin{array}{l}\text { BIPQ emotional } \\
\text { representation }\end{array}$ & 5.37 & $4.80-5.93$ & 6.00 & 3.18 & 0.00 & 10.00 & 6.00 \\
\hline BIPQ concern & 5.80 & $5.18-6.37$ & 6.00 & 3.21 & 0.00 & 10.00 & 6.00 \\
\hline BIPQ total & 16.32 & $14.64-17.93$ & 18.00 & 7.99 & 0.00 & 35.00 & 11.25 \\
\hline
\end{tabular}


Table 7.

Distribution of ISI Scores

\begin{tabular}{|c|c|c|c|}
\hline & $\%$ of sample & \% of males & $\%$ of females \\
\hline $\begin{array}{c}\text { None } \\
\text { (score 0-7) }\end{array}$ & 34.8 & 38.4 & 28.6 \\
\hline $\begin{array}{l}\text { Sub threshold } \\
\text { (score 8-14) }\end{array}$ & 30.4 & 31.4 & 28.6 \\
\hline $\begin{array}{c}\text { Moderate } \\
\text { (score 15-21) }\end{array}$ & 19.2 & 17.8 & 21.5 \\
\hline $\begin{array}{c}\text { Severe } \\
\text { (score 22-28) }\end{array}$ & 15.7 & 12.3 & 21.5 \\
\hline
\end{tabular}


Table 8 .

Distribution of DASS Scores

\begin{tabular}{|c|c|c|c|}
\hline & $\begin{array}{l}\text { Depression } \\
\text { (\% of sample) }\end{array}$ & $\begin{array}{c}\text { Anxiety } \\
\text { (\% of sample) }\end{array}$ & $\begin{array}{c}\text { Stress } \\
\text { (\% of sample) }\end{array}$ \\
\hline Normal & 82.6 & 80.9 & 90.4 \\
\hline Mild & 12.2 & 3.5 & 7 \\
\hline Moderate & 4.3 & 13.8 & 2.6 \\
\hline Severe & 0.9 & 0.9 & 0 \\
\hline Extremely severe & 0 & 0.9 & 0 \\
\hline
\end{tabular}

Note. DASS = Depression, Anxiety, Stress Scale.

The severity ratings are based on the recommended cut off scores (Lovibond \& Lovibond, 1995). Depression: normal (0-9), mild (10-13), moderate (14-20), severe (21-27), extremely severe $(28+)$. Anxiety: normal (0-7), mild (8-9), moderate (10-14), severe (15-19), extremely severe $(20+)$. Stress: normal (0-14), mild (15-19), moderate (19-25), severe (26-33), extremely severe $(37+)$. 
Table 9.

Factor Loadings for Principal Component Analysis with Varimax Rotation of the Brief Illness Perception Questionnaire

Scale $\quad$ Fatigue perception

BIPQ concern $\quad .91$

$\begin{array}{ll}\text { BIPQ consequences } & .90\end{array}$

BIPQ emotional representations $\quad .89$

BIPQ control $\quad .61$

Note. BIPQ = Brief Illness Perception Questionnaire 
Table 10.

Correlations Among Study Variables

\begin{tabular}{|c|c|c|c|c|c|c|c|c|c|c|c|}
\hline & ISI & DASS-D & DASS-A & $\begin{array}{c}\text { BIPQ } \\
\text { control }\end{array}$ & $\begin{array}{c}\text { BIPQ } \\
\text { Concern }\end{array}$ & $\begin{array}{c}\text { BIPQ } \\
\text { Emotion }\end{array}$ & $\begin{array}{l}\text { BIPQ } \\
\text { total }\end{array}$ & DISRS & CFAQ & $\begin{array}{l}\text { Number } \\
\text { of } \\
\text { medical } \\
\text { conditions }\end{array}$ & Fibrosis \\
\hline FSS & $.507 * *$ & $.514 * *$ & $.385 * *$ & $.505^{* *}$ & $.735 * *$ & $.656 * *$ & $.753 * *$ & $.642 * *$ & $-.569 * *$ & $.321 * *$ & .129 \\
\hline ISI & & $.425 * *$ & $.494 * *$ & $.305^{* *}$ & $.636^{* *}$ & .676 & $.656 * *$ & $.707 * *$ & $-.535 * *$ & $.308 * *$ & .159 \\
\hline DASS-D & & & $.627 * *$ & .186 & $.403 * *$ & $.452 * *$ & $.473 * *$ & $.643 * *$ & $-.525 * *$ & $.311 * *$ & .079 \\
\hline $\begin{array}{l}\text { BIPQ } \\
\text { control }\end{array}$ & & & & & $.399 * *$ & $.369 * *$ & $.619 * *$ & $.398 * *$ & $-.367 * *$ & .077 & .055 \\
\hline $\begin{array}{l}\text { BIPQ } \\
\text { concern }\end{array}$ & & & & & & $.753 * *$ & $.855 * *$ & $.631 * *$ & $-.588 * *$ & $.262 * *$ & .089 \\
\hline BIPQ & & & & & & & & & & & \\
\hline $\begin{array}{l}\text { emotional } \\
\text { representation }\end{array}$ & & & & & & & $.879 * *$ & $.687 * *$ & $-.590 * *$ & $.267 * *$ & .093 \\
\hline $\begin{array}{l}\text { Number } \\
\text { medical } \\
\text { conditions } \\
\text { Fibrosis }\end{array}$ & & & & & & & & & & & .147 \\
\hline
\end{tabular}

Note. The table presents the Spearman's rho coefficients for regression variables. FSS= Fatigue Severity Scale; ISI= Insomnia Severity Index; DASS-D= Depression Anxiety Stress Scale, Depression subscale; DASS-A= Depression Anxiety Stress Scale, Anxiety subscale; BIPQ control= Brief Illness Perception Questionnaire, Control item; BIPQ Concern = Brief Illness Perception Questionnaire, Concern item; BIPQ Emotion = Brief Illness Perception Questionnaire, Emotional representation item; DISRS = Daytime Insomnia Symptoms Response Scale; CFAQ = Chronic Fatigue Acceptance Questionnaire.

$* p<.05 * * p<.001$ 
Table 11.

Hierarchical Multiple Regression Models Predicting Fatigue

\begin{tabular}{|c|c|c|c|c|c|c|}
\hline Predictor & B & SE B & $\begin{array}{l}\text { Bias corrected } \\
\text { accelerated } \\
\text { 95\% CI for B }\end{array}$ & $ß$ & $\mathrm{R}^{2} \mathrm{adj}$ & $\mathrm{F}$ \\
\hline Step 1 & & & & & .054 & $3.85^{*}$ \\
\hline Fibrosis & .052 & .112 & $-.166-.259$ & .045 & & \\
\hline Total medical burden & .202 & .077 & $.039-.377$ & $.261 *$ & & \\
\hline
\end{tabular}

Step 2

$\begin{array}{lr}\text { Fibrosis } & -.00 \\ \text { Total medical burden } & .066 \\ \text { DASS-D } & .106 \\ \text { DASS-A } & -.019 \\ \text { ISI } & .08\end{array}$

$\begin{array}{llll}-.005 & .099 & -.202-.212 & -.004 \\ .066 & .077 & -.086-.203 & .085 \\ .106 & .051 & .002-.225 & .272^{*} \\ -.019 & .054 & -.119-.074 & -.042 \\ .083 & .022 & .041-.125 & .383^{* *}\end{array}$

Note. DASS-D= Depression Anxiety Stress Scale, Depression subscale; DASS-A= Depression Anxiety Stress Scale, Anxiety subscale; ISI= Insomnia Severity Index; BIPQ = Brief Illness Perception Questionnaire, DISRS = Daytime Insomnia Symptoms Response Scale; CFAQ = Chronic Fatigue Acceptance Questionnaire.

$* p<.05 * * p<.001$ 
Table 11.

Hierarchical Multiple Regression Models Predicting Fatigue (continued from previous page)

\begin{tabular}{|c|c|c|c|c|c|c|}
\hline Predictor & B & SE B & $\begin{array}{l}\text { Bias corrected } \\
\text { accelerated } \\
95 \% \text { CI for B }\end{array}$ & $ß$ & $\mathrm{R}^{2} \mathrm{adj}$ & $\mathrm{F}$ \\
\hline Step 3 & & & & & .521 & $14.57 * *$ \\
\hline Fibrosis & .022 & .081 & $-.142-.212$ & .019 & & \\
\hline Total medical burden & .082 & .062 & $-.037-.185$ & .106 & & \\
\hline DASS-D & -.002 & .044 & $-.090-.106$ & -.006 & & \\
\hline DASS-A & -.003 & .046 & $-.089-.069$ & -.007 & & \\
\hline ISI & -.022 & .021 & $-.066-.017$ & -.101 & & \\
\hline BIPQ & .101 & .027 & $.054-.154$ & $.518 * *$ & & \\
\hline CFAQ & -.027 & .008 & $-.031-.003$ & -.160 & & \\
\hline DISRS & .027 & .017 & $-.005-.062$ & .226 & & \\
\hline
\end{tabular}

Note. DASS-D= Depression Anxiety Stress Scale, Depression subscale; DASS-A= Depression Anxiety Stress Scale, Anxiety subscale; ISI= Insomnia Severity Index; BIPQ = Brief Illness Perception Questionnaire, DISRS = Daytime Insomnia Symptoms Response Scale; CFAQ = Chronic Fatigue Acceptance Questionnaire.

$* p<.05 * * p<.001$ 
Table 12.

Final Hierarchical Multiple Regression Model Predicting Fatigue

\begin{tabular}{|c|c|c|c|c|c|c|}
\hline Predictor & B & SE B & $\begin{array}{l}\text { Bias corrected } \\
\text { accelerated } \\
95 \% \text { CI for B }\end{array}$ & $ß$ & $\mathrm{R}^{2} \mathrm{adj}$ & F \\
\hline Step 1 & & & & & .304 & $16.414^{* *}$ \\
\hline $\begin{array}{l}\text { Number of medical } \\
\text { conditions }\end{array}$ & .067 & .074 & $-.086-.211$ & .087 & & \\
\hline DASS-D & .108 & .035 & .038-.183 & $.281 * *$ & & \\
\hline ISI & .077 & .020 & $.037-.116$ & $.350 * *$ & & \\
\hline Step 2 & & & & & .484 & $25.871^{* *}$ \\
\hline $\begin{array}{l}\text { Number of medical } \\
\text { conditions }\end{array}$ & .096 & .058 & $-.023-.208$ & .124 & & \\
\hline DASS-D & .063 & .031 & $.006-.133$ & $.163^{*}$ & & \\
\hline ISI & .001 & .018 & $-.036-.035$ & .004 & & \\
\hline BIPQ & .116 & .024 & $.070-.163$ & $.582 * *$ & & \\
\hline
\end{tabular}

Note. DASS-D= Depression Anxiety Stress Scale, depression subscale; ISI= Insomnia Severity Index; BIPQ = Brief Illness Perception Questionnaire

$* p<.05 * * p<.001$ 
Table 13.

Results of the Exploratory Mediation Analyses

\begin{tabular}{|c|c|c|c|c|c|c|c|}
\hline Predictor & Mediator & Outcome & $\begin{array}{l}\text { Total effect of } \\
\text { predictor on } \\
\text { outcome } \\
\text { (c path) }\end{array}$ & $\begin{array}{l}\text { Direct effect of } \\
\text { predictor on } \\
\text { outcome } \\
\text { ( c’ path) }\end{array}$ & $\begin{array}{c}\text { Indirect effect } \\
\text { of } \\
\text { predictor } \\
\text { on outcome } \\
\text { (a x b path) }\end{array}$ & $\begin{array}{c}\text { Completely } \\
\text { standardized } \\
\text { indirect effect }\end{array}$ & $\begin{array}{c}\text { Completely } \\
\text { standardized } \\
\text { indirect effect } \\
95 \% \text { bias } \\
\text { corrected CI }\end{array}$ \\
\hline \multirow[t]{3}{*}{ DASS-D } & & FSS & $.175 * * *$ & $.065 *$ & .109 & .285 & .160-.407 \\
\hline & BIPQ concern & & & & .091 & .238 & $.128-.371$ \\
\hline & BIPQ control & & & & .018 & .047 & $.004-.130$ \\
\hline \multirow[t]{3}{*}{ ISI } & & FSS & $.115^{* * *}$ & .013 & .102 & .465 & $.336-.589$ \\
\hline & BIPQ concern & & & & .082 & .376 & $.244-.513$ \\
\hline & BIPQ control & & & & .020 & .089 & $.029-.197$ \\
\hline \multirow{3}{*}{$\begin{array}{l}\text { Number of } \\
\text { medical } \\
\text { conditions }\end{array}$} & & FSS & $.258^{* *}$ & $.098 *$ & .160 & .215 & $.066-.345$ \\
\hline & BIPQ concern & & & & .138 & .185 & $.076-.310$ \\
\hline & BIPQ control & & & & .022 & .030 & $-.022-.108$ \\
\hline
\end{tabular}


Table 14.

Differences in Fatigue and Sleepiness in Insomnia and Sleep Apnea Groups

\section{Sleep Disorder Groups}

\begin{tabular}{lcccccc}
\cline { 2 - 5 } & $\begin{array}{c}\text { No clinical } \\
\text { insomnia } \\
\text { or OSA } \\
\text { reported }\end{array}$ & Insomnia & OSA & $\begin{array}{c}\text { Insomnia } \\
\text { and OSA }\end{array}$ & $\mathrm{F}$ & $\mathrm{p}$ \\
\hline $\begin{array}{l}\text { Fatigue } \\
(\text { FSS })\end{array}$ & $3.71_{\mathrm{a}}$ & $4.98_{\mathrm{b}}$ & $3.89_{\mathrm{a}}$ & $5.53_{\mathrm{b}}$ & 7.79 & .001 \\
$\begin{array}{l}\text { Sleepiness } \\
(\text { ESS })\end{array}$ & $7.8_{\mathrm{a}}$ & $10.75 \mathrm{a}_{\mathrm{b}}$ & $9.65 \mathrm{a}_{\mathrm{b}}$ & $12.69_{\mathrm{b}}$ & 4.86 & .001 \\
\hline
\end{tabular}

Note. Means with different subscripts in a row are significantly different at $p<.05$ based on Hochberg and Games-Howell post hoc tests. FSS= Fatigue Severity Scale; ISI= Insomnia Severity Index; OSA= obstructive sleep apnea. 
Table 15.

Qualitative Themes

\begin{tabular}{|c|c|c|}
\hline Themes & $\mathrm{N}$ & $\%$ \\
\hline \multicolumn{3}{|l|}{ Characteristics } \\
\hline Physical & 10 & 71.4 \\
\hline Emotion as a consequence & 8 & 57.1 \\
\hline Cognitive & 6 & 42.9 \\
\hline Persistent & 6 & 42.9 \\
\hline Sudden feeling of complete exhaustion & 4 & 28.6 \\
\hline Starts early & 3 & 21.4 \\
\hline \multicolumn{3}{|l|}{ Course } \\
\hline Chronic progressive & 6 & 42.9 \\
\hline Chronic stable & 3 & 21.4 \\
\hline \multicolumn{3}{|l|}{ Triggers } \\
\hline Activity & 11 & 78.6 \\
\hline Sleep & 8 & 57.1 \\
\hline Stress & 6 & 42.9 \\
\hline HCV related anxiety & 5 & 35.7 \\
\hline \multicolumn{3}{|l|}{ Cognitive } \\
\hline \multicolumn{3}{|l|}{ Attributions } \\
\hline HCV infection & 12 & 85.7 \\
\hline Other medical conditions & 5 & 35.7 \\
\hline Sleep disorder/chronic sleep problem caused & 3 & 21.4 \\
\hline \multicolumn{3}{|l|}{ Other cognitions } \\
\hline Control & 11 & 78.6 \\
\hline Prognosis & 11 & 78.6 \\
\hline Acceptance & 9 & 64.3 \\
\hline Positive attitude & 8 & 57.1 \\
\hline Cognitive avoidance & 6 & 42.9 \\
\hline \multicolumn{3}{|l|}{ Fatigue related behaviours } \\
\hline Non-active rest & 10 & 71.4 \\
\hline Sleep & 9 & 64.3 \\
\hline Active rest & 8 & 57.1 \\
\hline Physical activity & 8 & 57.1 \\
\hline Nutrition & 9 & 64.3 \\
\hline Pacing & 9 & 64.3 \\
\hline Fatigue driven activity & 7 & 50.0 \\
\hline Pleasurable activities & 5 & 35.7 \\
\hline \multicolumn{3}{|l|}{ Functional impact of fatigue } \\
\hline Family life and intimate relationships & 10 & 71.4 \\
\hline Physical functioning & 9 & 64.3 \\
\hline Work & 9 & 64.3 \\
\hline Social life & 9 & 64.3 \\
\hline Active recreation & 7 & 50.0 \\
\hline Future plans & 6 & 42.9 \\
\hline Finances & 4 & 28.6 \\
\hline
\end{tabular}


Table 15.

Qualitative Themes (continued)

Themes

$\mathrm{N}$

$\%$

Communication with health care providers

Specialist

8

57.1

Family physician

7

50.0

Reasons for not communicating with health care providers

8

57.1

Note. $\mathrm{N}=$ the number of participants who reported at least one instance in the sub-category. $\%=$ the percentage of the total sample. The sum of $\mathrm{N}$ can be larger than 14 and the sum of \% can be larger than 100 in the main themes, since a participant's response could have included more than one sub-category within the theme. 
Table 16.

The Most Important Fatigue Attributions

\begin{tabular}{|c|c|c|c|}
\hline \multicolumn{2}{|c|}{ High fatigue (FSS $\geq 4$ ) } & \multicolumn{2}{|c|}{ Low fatigue $(\mathrm{FSS}<4)$} \\
\hline $\mathrm{HCV}$ & (37\%) & Sleep & $(19 \%)$ \\
\hline Sleep & $(13 \%)$ & $\begin{array}{c}\text { Other } \\
\text { medical } \\
\text { conditions }\end{array}$ & $(16 \%)$ \\
\hline $\begin{array}{l}\text { Other } \\
\text { medical }\end{array}$ & $(13 \%)$ & $\begin{array}{l}\text { Physical } \\
\text { activity }\end{array}$ & $(16 \%)$ \\
\hline & & Stress & $(16 \%)$ \\
\hline
\end{tabular}

Note. The table shows the factors that patients named as the most important causes of their fatigue on the BIPQ. The numbers in brackets indicate the percentage of total attributions. FSS $=$ Fatigue Severity Scale 
Figure 1. Conceptual Framework for Understanding Fatigue

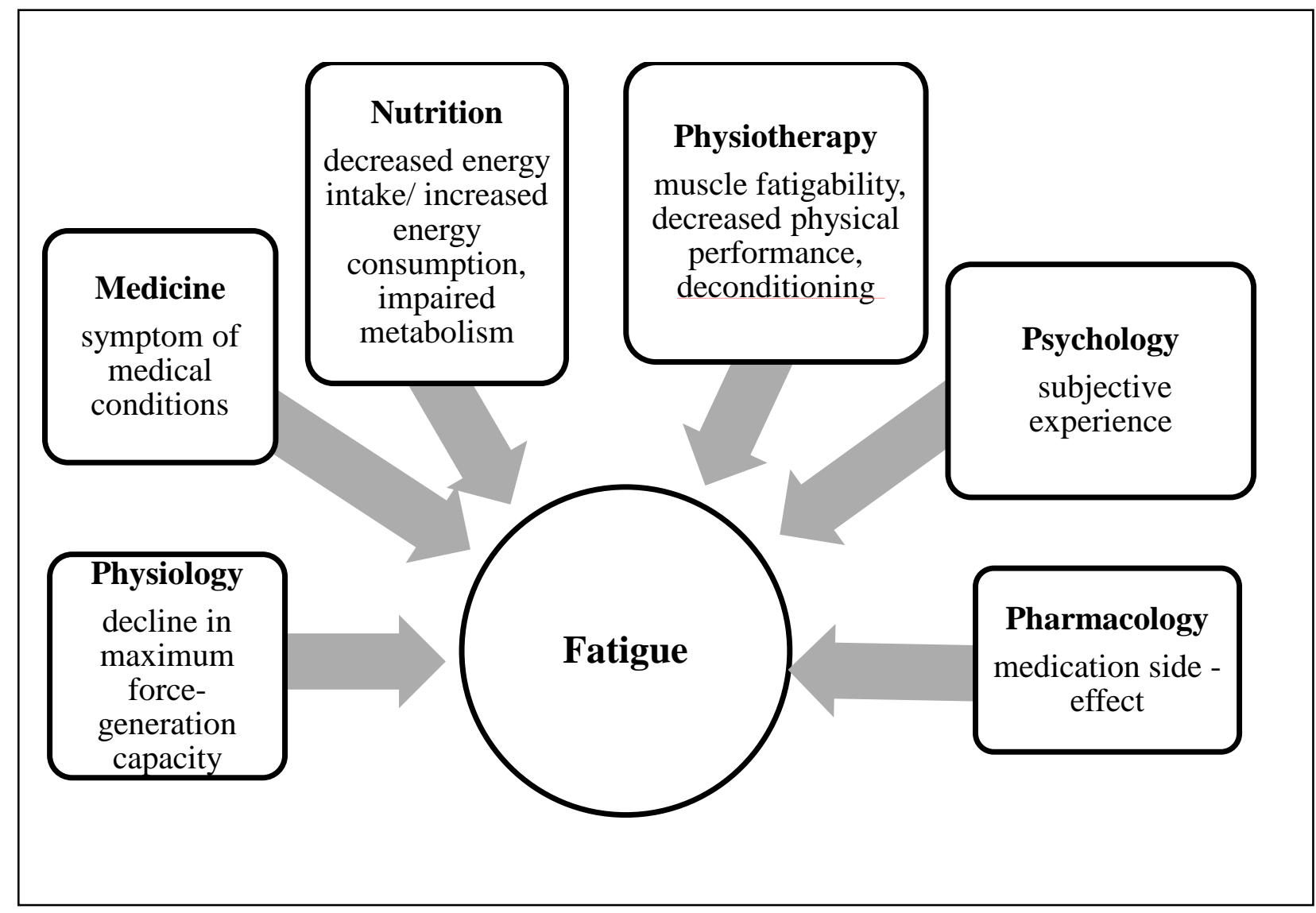


Figure 2. The Two Stage Model of Fatigue in Medical Conditions

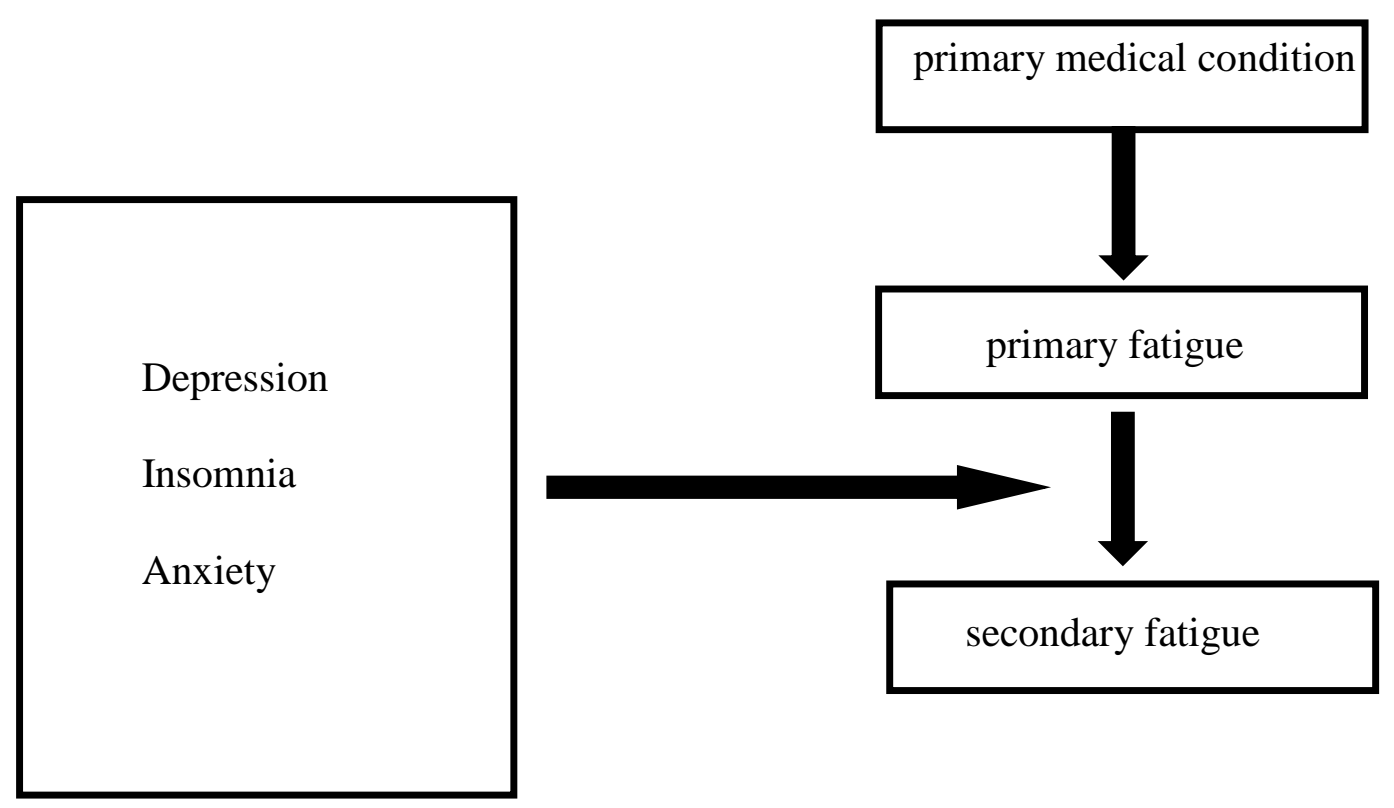


Figure 3. The Scatterplot of Fatigue (FSS) and Sleepiness (ESS) Scores

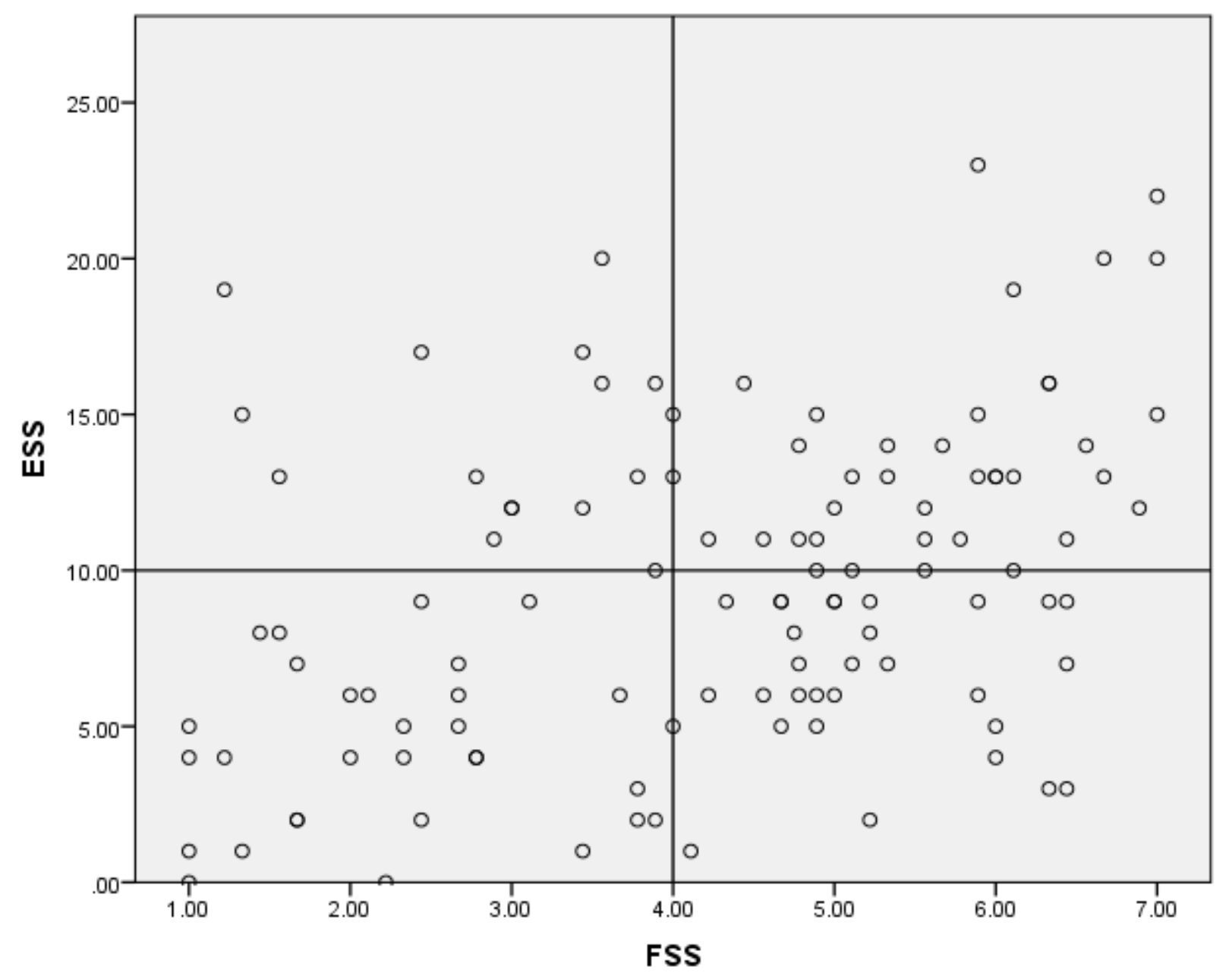

Figure 3. The figure displays the scatter plot of the fatigue (FSS) and subjective sleepiness (ESS) scores. The cut-off scores of the questionnaires divide the sample into four groups: only high fatigue; only high sleepiness; both high fatigue and sleepiness and neither high fatigue or high sleepiness. FSS = Fatigue Severity Scale, ESS = Epworth Sleepiness Scale 
Figure 4. The Results of the Survey Question on Possible Personal Benefits of a Fatigue Management Service at the Liver Clinic

a.

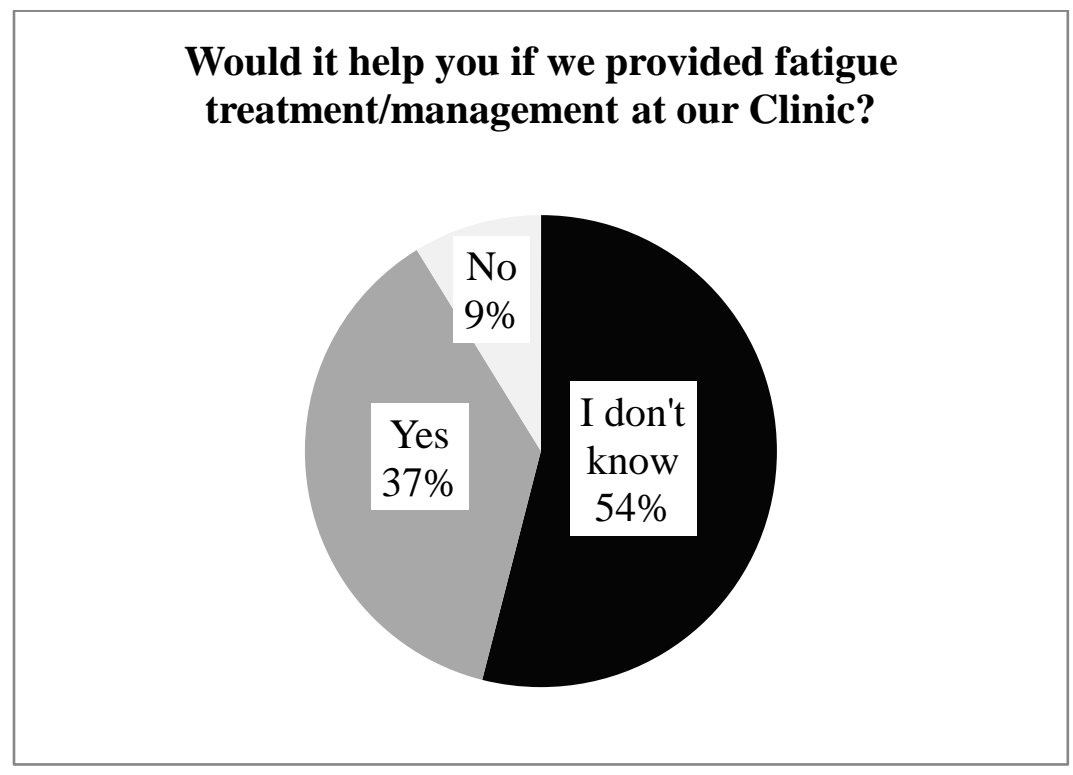

b.

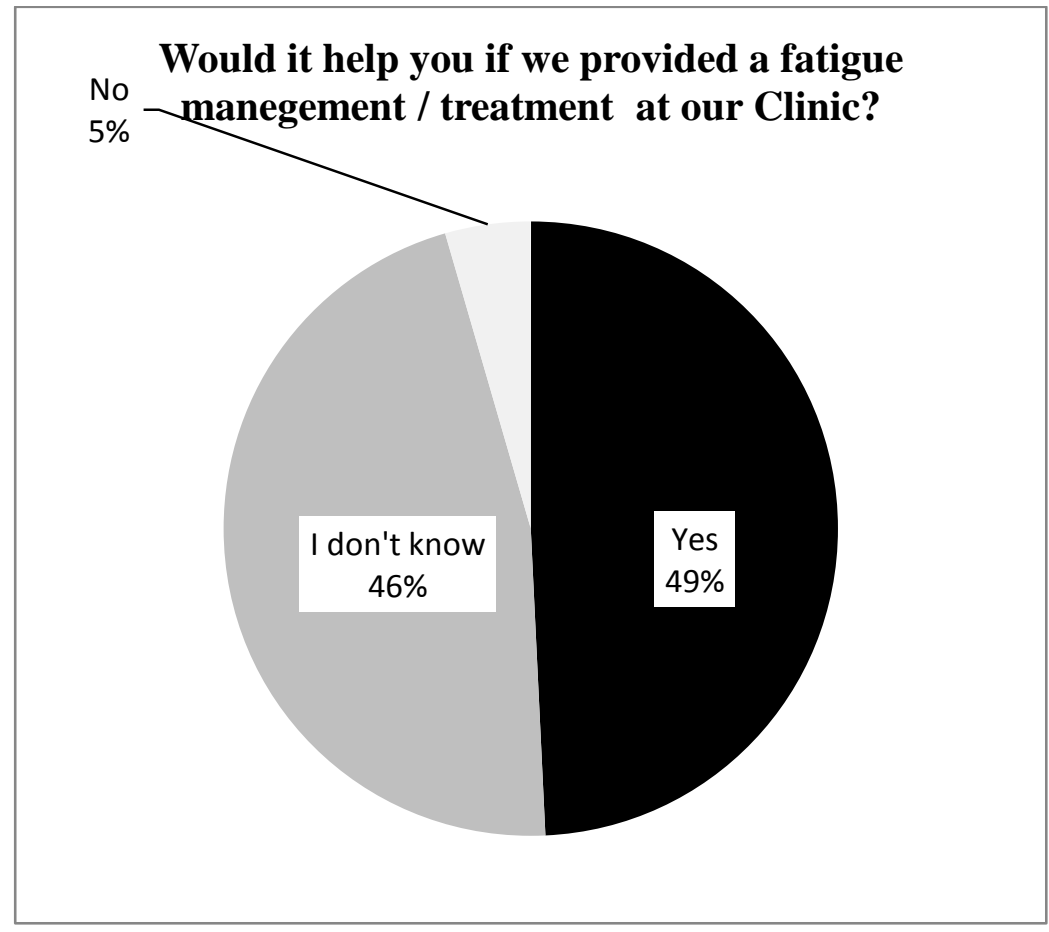

Figure 4. (a) total sample (b) patients with severe fatigue outcomes (FSS $\geq 4$ ) 
Figure 5. The Distribution of STOP-BANG Scores

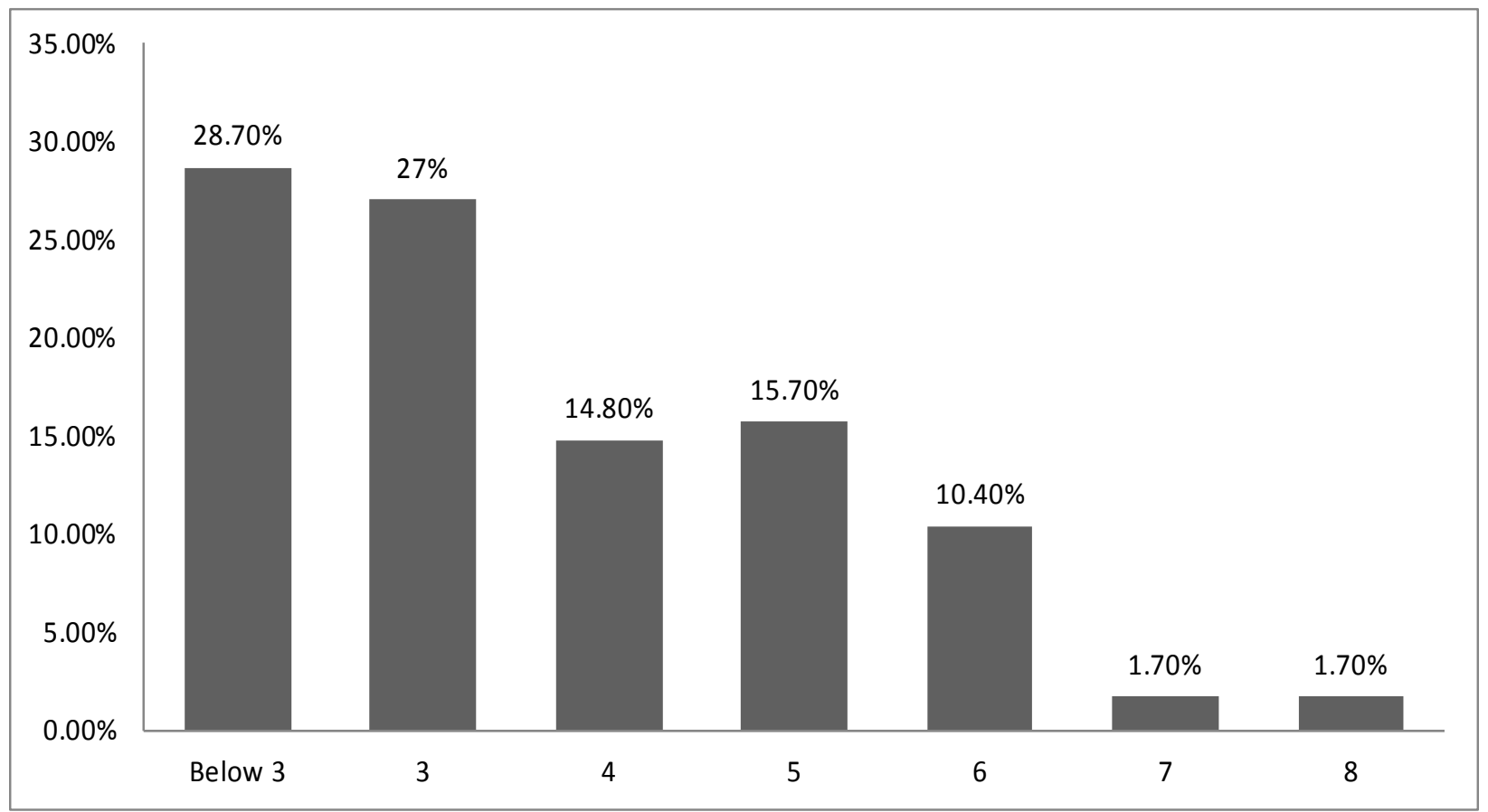

Figure 5. The minimum score is 0 and the maximum score is 8 . The suggested cut-off score for screening is 3 . The suggested cut-off to screen for moderate or severe sleep apnea is 5 . Scores indicating moderate or severe sleep apnea (scores 5-8) were obtained by $29.5 \%$ of the sample. 
Figure 6. General Mediation Model with Multiple Mediators.

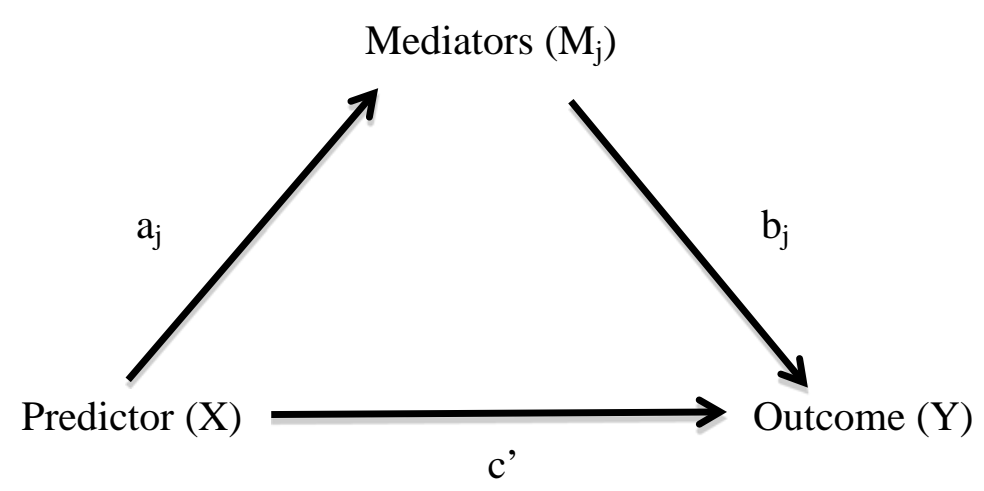

Total effect $(\mathbf{c})=\operatorname{direct}$ effect $\left(c^{\prime}\right)+\sum_{\mathbf{j}}\left(\mathbf{a}_{1 \mathbf{j}} \mathbf{b}_{1 \mathbf{j}}\right)$

Figure 6. The figure illustrates that the total effect a predictor $(\mathrm{X})$ on an outcome $(\mathrm{Y})$ can have two constituents: a direct effect and an indirect effect. When a direct effect occurs, the changes in $\mathrm{X}$ cause changes in Y directly (path c'). The indirect effect happens when changes in X cause changes in $\mathrm{Y}$ through changes in intervening variables or mediators (M). The indirect effect of $\mathrm{X}$ on $\mathrm{Y}$ is therefore the sum of the products of " $\mathrm{a}$ " (the effect $\mathrm{X}$ on $\mathrm{M}$ ) and $\mathrm{b}$ (the effect of $\mathrm{M}$ on Y after controlling for c'). 
Figure 7. Mediation Model 1

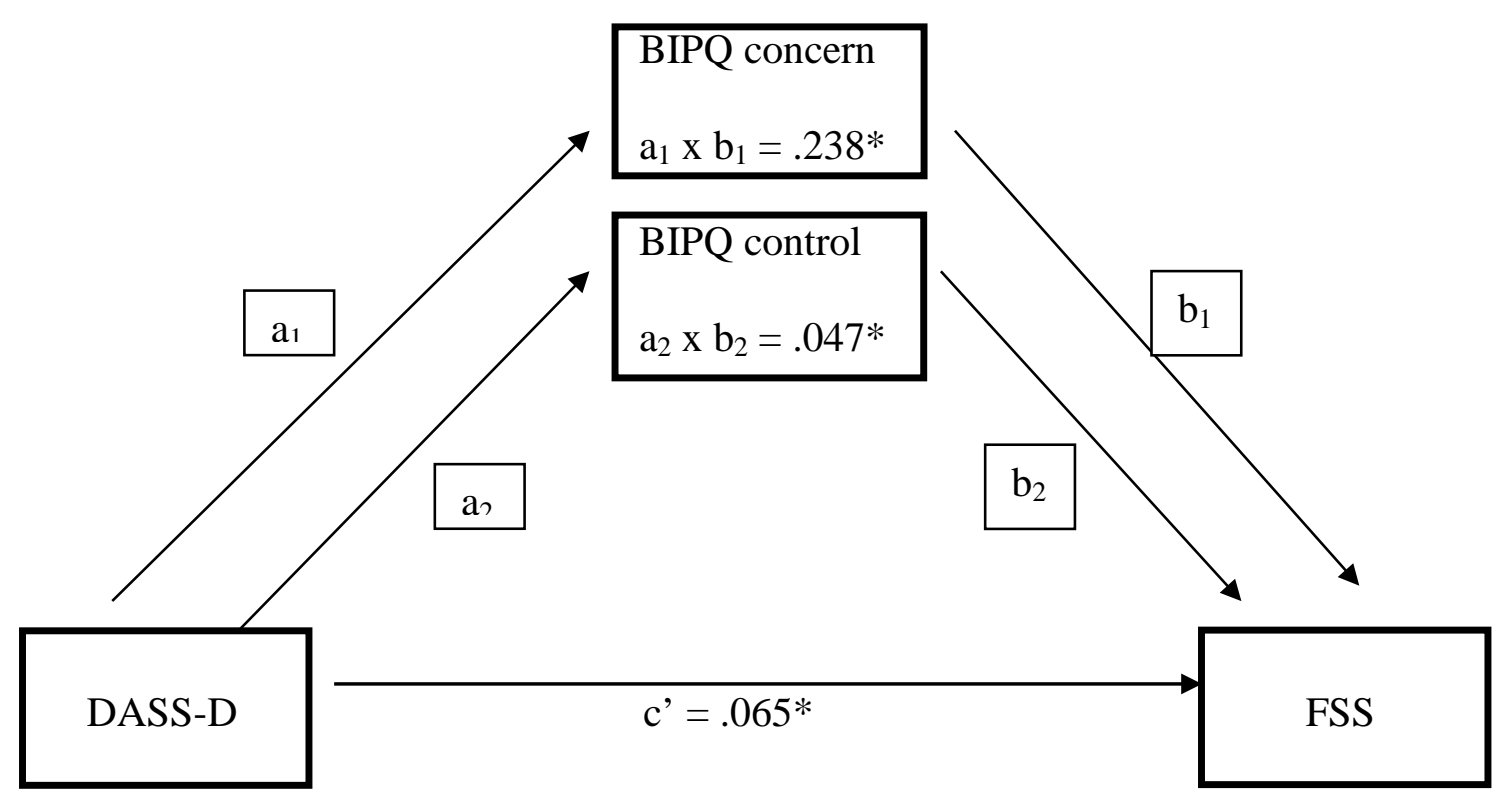

Total effect $(\mathrm{c})=.175^{*}$

Figure 7. The mediated effect of DASS-D on FSS through the BIPQ. a $\mathrm{x} b=$ totally standardized indirect effects. c and c' are not standardized. * denotes statistically significant results. DASS$\mathrm{D}=$ Depression, Anxiety, Stress Scale- Depression subscale. BIPQ = Brief Illness Perception Questionnaire, FSS = Fatigue Severity Scale 
Figure 8. Mediation Model 2

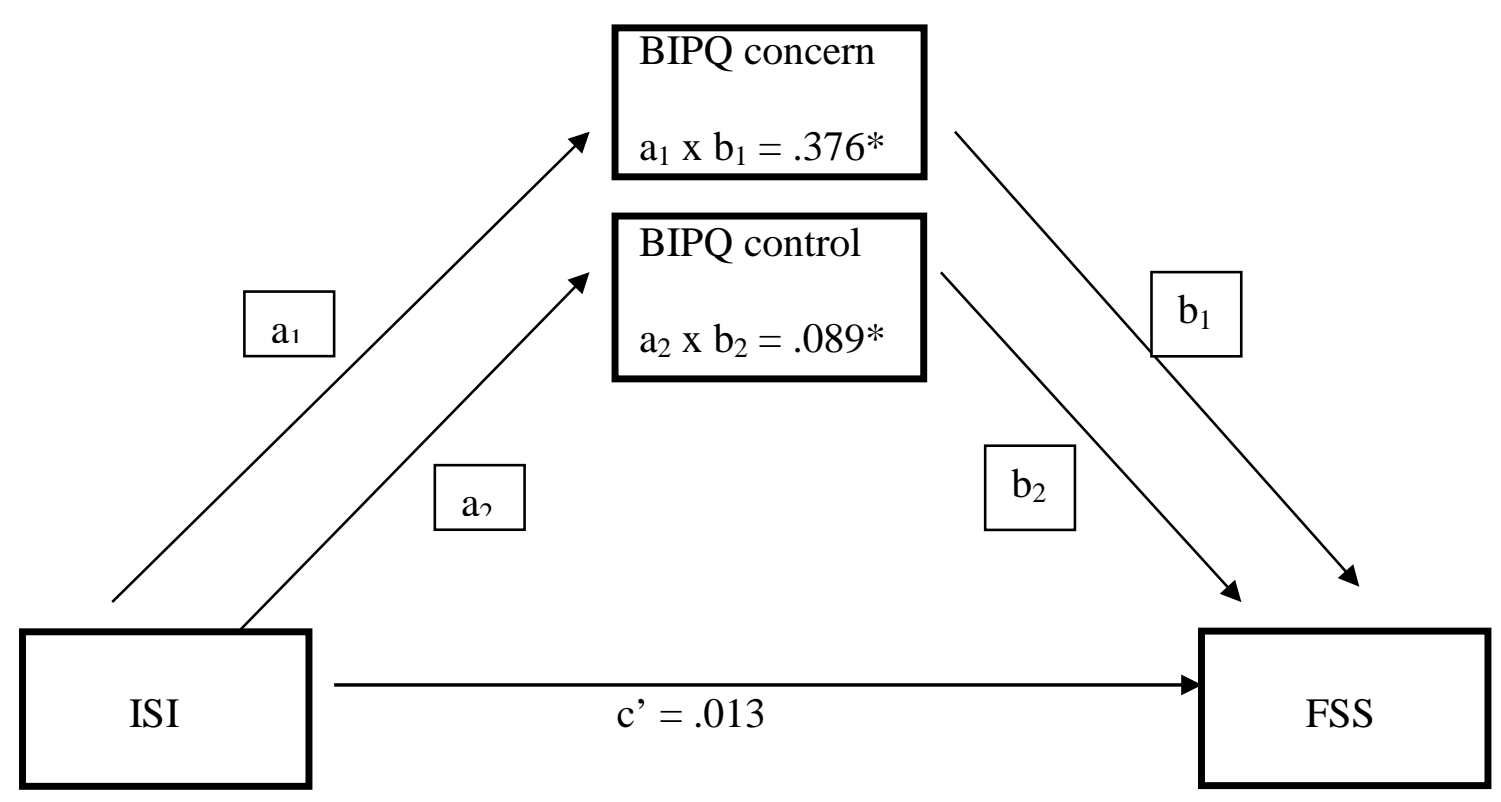

Total effect $(\mathrm{c})=.115^{*}$

Figure 8. The mediated effect of ISI on FSS through the BIPQ. a $\mathrm{x} b=$ totally standardized indirect effects. c and c' are not standardized. * denotes statistically significant results. ISI= Insomnia Severity Index, BIPQ = Brief Illness Perception Questionnaire, FSS = Fatigue Severity Scale 
Figure 9. Mediation Model 3

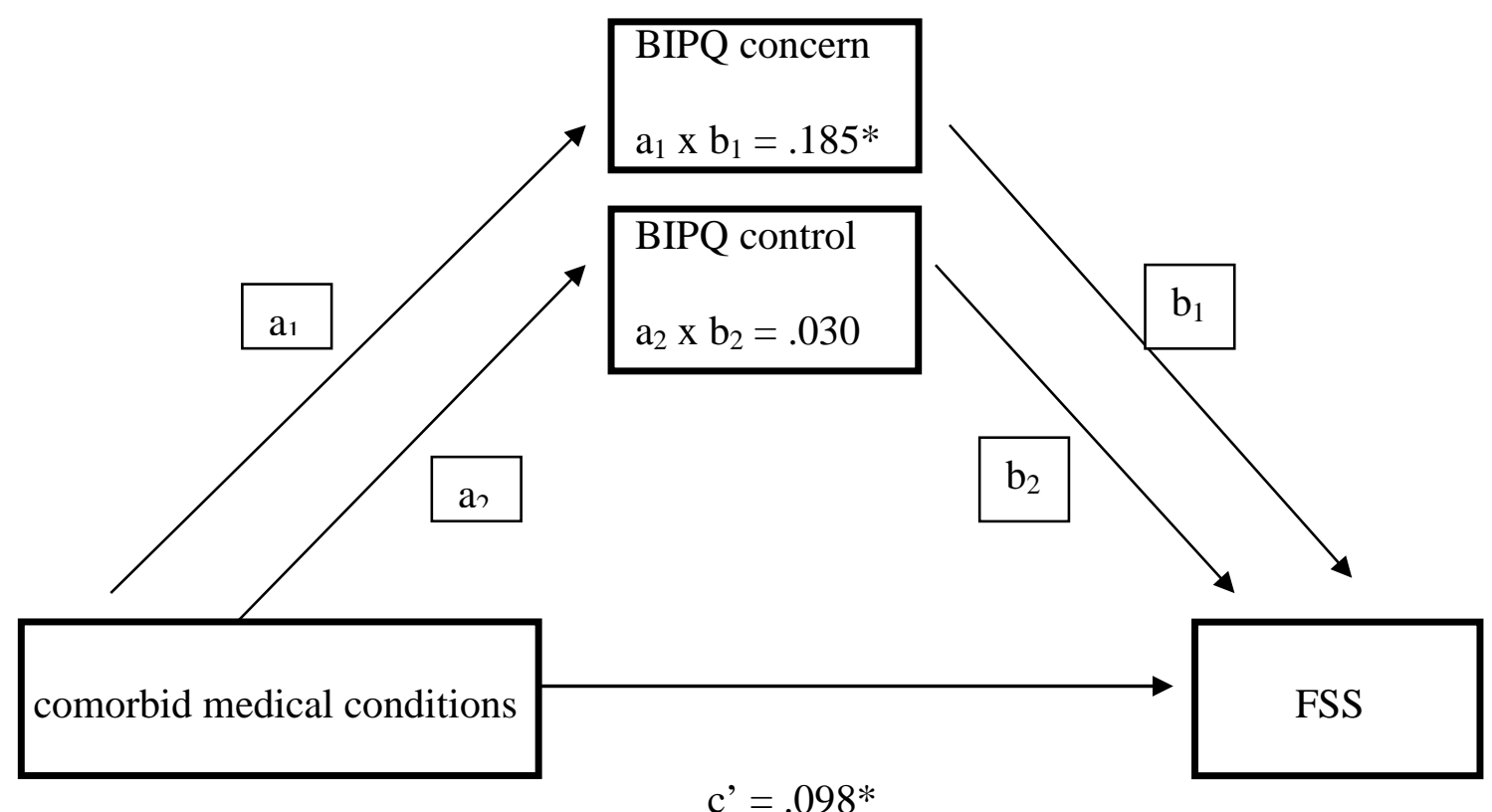

Total effect $(\mathrm{c})=.258 *$

Figure 9. The mediated effect of comorbid medical conditions on FSS through the BIPQ. $\mathrm{a} \times \mathrm{b}=$ totally standardized indirect effects. c and c' are not standardized. * denotes statistically significant results. FSS = Fatigue Severity Scale; BIPQ = Brief Illness Perception Questionnaire 
Figure10. Insomnia and Obstructive Sleep Apnea (OSA) Screening Results

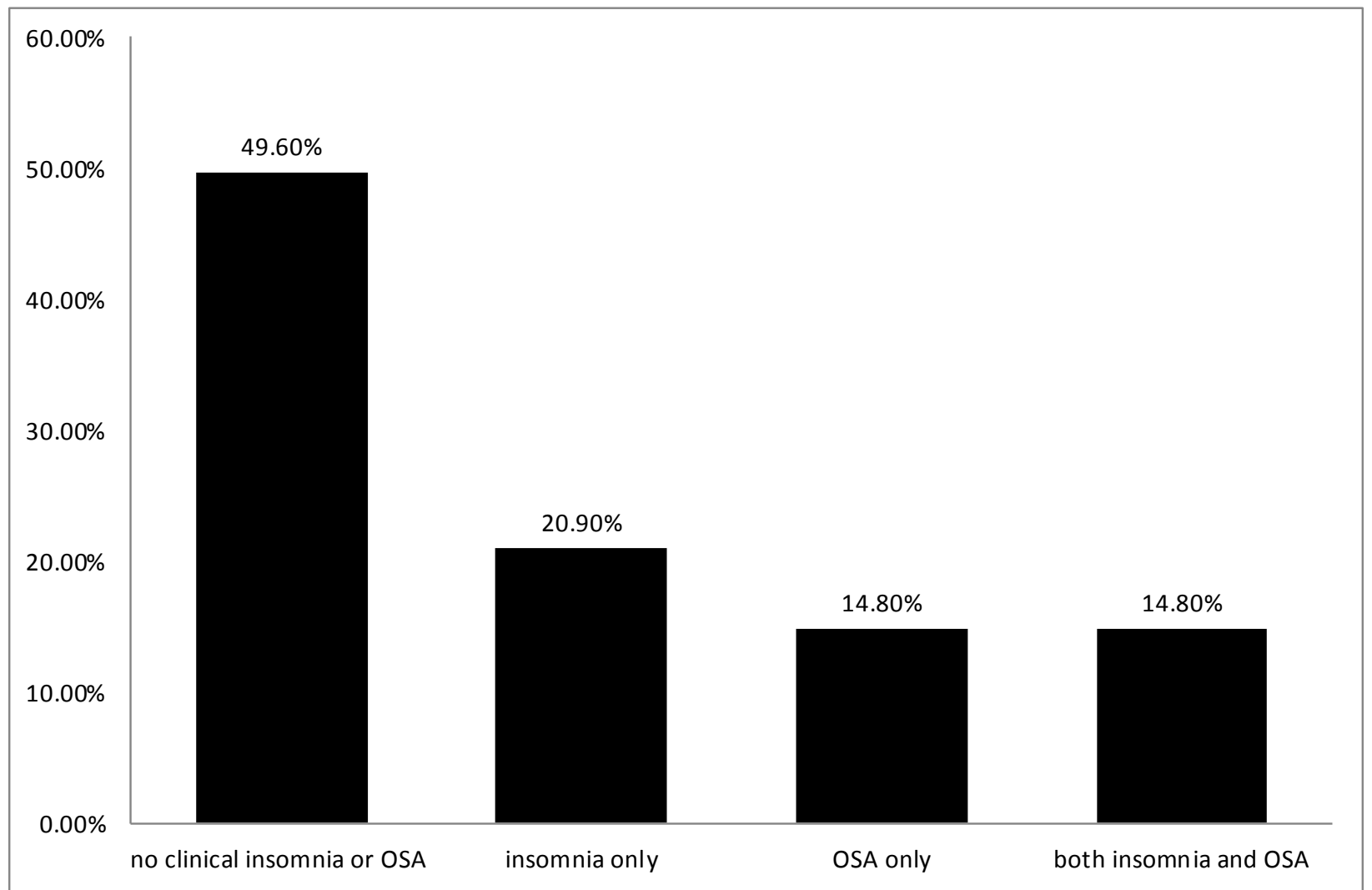

Figure 10. The figure illustrates the percentage of participants in the following groups: (a) no sleep problem (ISI $\leq 14$ and STOP-BANG $<5$ ); (b) insomnia only (ISI $>14$ and STOP-BANG < 5); (c) OSA only (ISI $\leq 14$ and STOP-BANG $\geq 5$ ), and (d) both insomnia and OSA (ISI $>14$ and STOP-BANG $\geq 5$ ). 
Figure11. Fatigue Perception Model

\section{FATIGUE IS EXPERIENCED IN AN ILLNESS ENVIRONMENT}

(e.g. liver disease, heart disease, kidney disease, cancer, insomnia, depression)

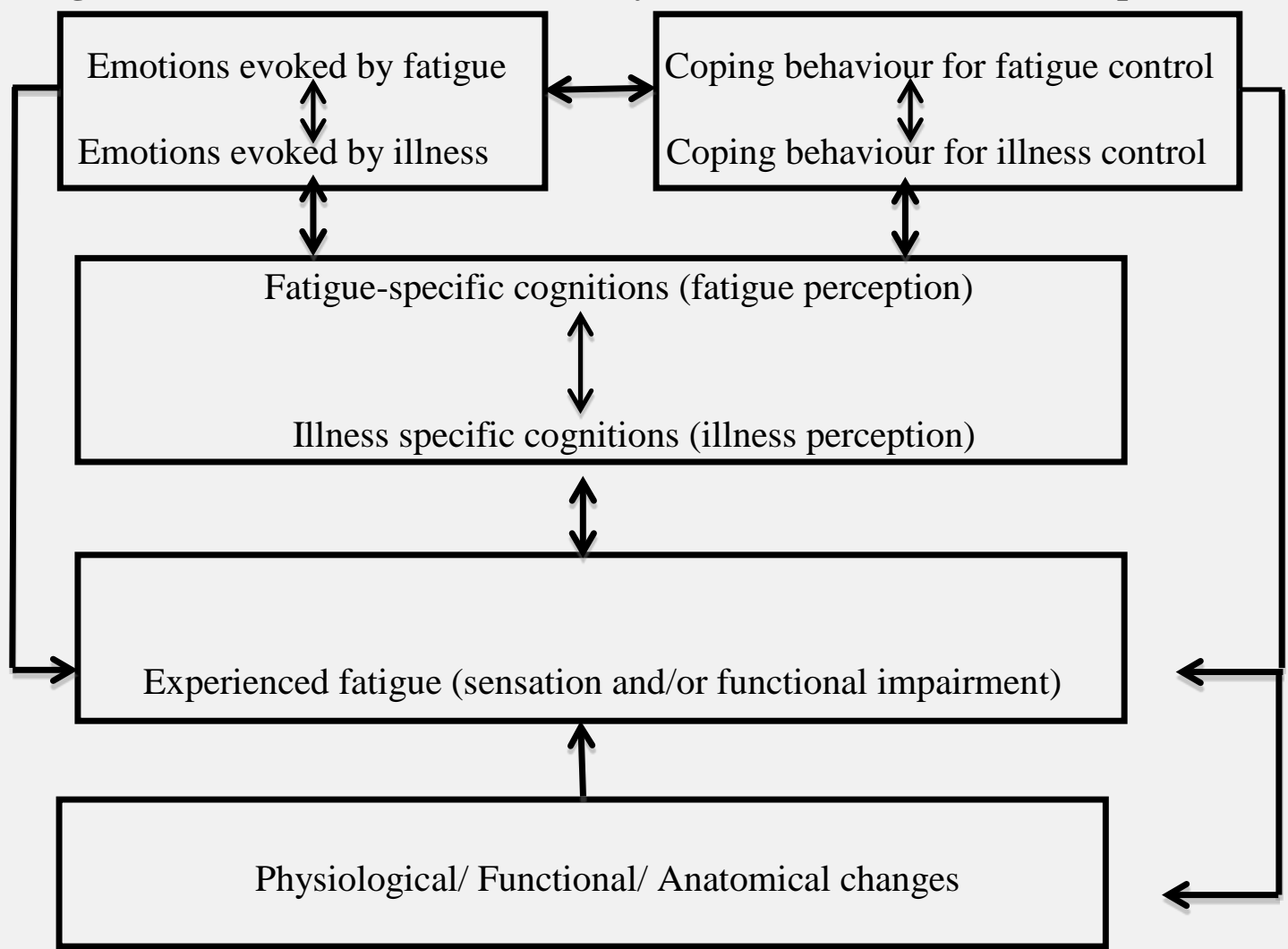

Figure 11. The model depicts that physiological, cognitive, behavioural and emotional factors contribute to the experience of fatigue in chronic medical conditions. Illness perceptions, behaviours to cope with the illness and emotional illness representations interact with fatiguespecific cognitions, fatigue management behaviours and with emotions evoked by the fatigue. This could explain, in part, the observed differences in fatigue cognitions/behaviours in different medical conditions. 


\section{Appendix A: Commonly Used Fatigue Questionnaires}

Below is a short summary of commonly used fatigue questionnaires. Summary tables, including the psychometric properties are found in (Dittner, et al., 2004) and in (Elbers et al., 2012) . The questionnaires are re-printed in Shahid et al, 2011.

\section{Fatigue Severity Scale}

The Fatigue Severity Scale (FSS) is one the most popular fatigue scales developed for the assessment of the functional outcomes of fatigue (Krupp, et al., 1989a). Respondents indicate their strength of agreement with the nine items of the scale from 1 (strongly disagree) to 7 (strongly agree) referring to the past week or past two weeks. The fatigue score is the mean score of the 9 items. The scale was first validated in lupus erythematosus and multiple sclerosis and showed an internal consistency of .88 and test-retest reliability of .84 (Krupp, et al., 1989a). The FSS has been used in research studies to assess fatigue of patients with multiple sclerosis, cancer, sleep disorders, chronic fatigue syndrome, major depressive disorders, Parkinson's disease, brain injury, amyothropic lateral sclerosis and chronic HCV infection (Dittner, et al., 2004). The FSS can differentiate between fatigue outcomes reported by samples with major depression, chronic fatigue syndrome and multiple sclerosis (Pepper, et al., 1993) The FSS was more strongly associated with the symptoms and the patient reported functional outcomes of chronic fatigue syndrome than the Chalder Fatigue Scale (Taylor, et al., 2000). Based on the latter findings, it was concluded that the FSS is a more appropriate measure of the functional outcomes of chronic fatigue and of fatigue-related disability. Interestingly, one study concerning multiple sclerosis found that the FSS was not precise measuring severe fatigue, and the authors suggested that the Modified Fatigue Impact Scale is used for the assessment of severe fatigue in this population (Amtmann et al., 2012).(Lee, et al., 1991) The FSS showed excellent psychometric properties 
(internal consistency $\alpha=.94$; test-retest reliability during an average of 25 days interval $=.82$, convergent validity with SF-36 vitality subscale $r=-0.76$ ) in a multi-center clinical trial, wherein fatigue was assessed at screening and baseline before initiation of a combined antiviral treatment in chronic HCV infection (Kleinman, et al., 2000). The pre-treatment score was 3.8 in the latter sample. It has been suggested that clinical trials use the FSS as a patient reported outcome measure in chronic HCV infection. The questionnaire measures functional impairment, it is sensitive to change and it has excellent psychometric properties in this population (Kleinman, et al., 2012).

\section{Chalder Fatigue Scale}

As opposed to the FSS that measures fatigue outcomes, the Chalder Fatigue Scale (CFS) (also called Fatigue Scale or FS) assesses the intensity of fatigue. The CFS was developed to measure fatigue in chronic fatigue syndrome (Chalder, et al., 1993). The CFS has 11 items, each with four response choices ranging from 1 (much worse than usual) to 4 (better than usual). The items load into "physical fatigue" and "mental fatigue" dimensions. There are two scoring options: either summing the scores or assigning a value of "one" to choices 1 and 2 and assigning a value of "two" to response choices 3 and 4 on each item (bimodal scoring) before adding the scores. The suggested cut-off for pathological fatigue (based on the bimodal scoring) is three of four (Shahid, et al., 2011). The questionnaire has been validated in the general population ( $\alpha=.88$ .90 ) and subsequently has been used in medical populations, for example primary care patients, patients with chronic fatigue syndrome, cancer, post-polio syndrome and multiple sclerosis (Armes, Chalder, Addington-Hall, Richardson, \& Hotopf, 2007; Ferrando et al., 1998; Wessely, Chalder, Hirsch, Wallace, \& Wright, 1997). The CFS is sensitive to treatment related change; therefore it has been popular in CBT trials in chronic fatigue syndrome and multiple sclerosis 
(van Kessel, et al., 2008; White, et al., 2011). As noted above, the CFS is less able to capture fatigue outcomes than the FSS does in chronic fatigue syndrome (Taylor, et al., 2000). Another disadvantage of the CFS is the lack of a specific recall period. Finally, it contains an item on sleepiness, which might compromise its construct validity.

\section{Multidimensional Fatigue Inventory}

The Multidimensional Fatigue Inventory (MFI-20) was developed to provide a short, multidimensional fatigue assessment tool for cancer patients (Smets, et al., 1995). In the original validation study four scale and five scale structures appeared to be equally acceptable, but the authors suggested that the full, five dimension - questionnaire is used until subsequent studies provide further data on the construct validity of the questionnaire. The five scales in the validation study were: general fatigue; physical fatigue; mental fatigue; reduced activity and reduced motivation. Each of the five scales consists of four questionnaire items. Respondents indicate on a 5-point Likert scale whether they agree (yes, this is true) or disagree (no that is not true) with the statements. There have been concerns about the construct validity of the questionnaire based on both in the original and in subsequent studies (Dittner, et al., 2004). In the original paper, for example the cancer patients reported the lowest mental fatigue scores among the samples assessed (radiotherapy patients, medical students, psychology students, army recruits, junior physicians, chronic fatigue patients) (Smets, et al., 1995). Also, the students and the cancer patients reported similar degree of "general fatigue”. In subsequent studies in patients with cancer and Parkinson's disease the five scale structure was not confirmed (Elbers, van Wegen, Verhoef, \& Kwakkel, 2012; Fillion, Gelinas, Simard, Savard, \& Gagnon, 2003; Gentile, Delaroziere, Favre, Sambuc, \& San Marco, 2003). The issue was raised that if, following the developers' recommendations, the general fatigue and the physical fatigue subscales are merged 
to create a four-dimension questionnaire, then it is not clear what the new dimension would measure (Elbers, van Wegen, et al., 2012). A related, pragmatic issue is that the scoring is based on the five dimension structure. A further problem with the questionnaire structure has been that some items have not had stable factor loadings (some items have loaded to different scales in different studies). Nevertheless, the questionnaire has been a recommended for screening and assessment of fatigue in Parkinson’s disease (Friedman et al., 2010).

\section{Fatigue Impact Scale}

The Fatigue Impact Scale (FIS) was developed for use in medical conditions with chronic fatigue (Fisk, et al., 1994). The questionnaire was designed to assess the impact of fatigue (rather than fatigue intensity). The questionnaire items were generated based on multiple sclerosis patients’ description on fatigue outcomes. The FIS has 40 items that load into three sub-scales (physical, cognitive and social). The responses are measured in a 5 point Likert scale with values ranging

from 0 to 4 . The recall period is one month. In the original validation study the FIS showed high internal consistency ( $\alpha=.93$ ) and it discriminated between patients with multiple sclerosis and hypertension (Fisk, et al., 1994). Subsequently, FIS was used to assess fatigue impact in chronic fatigue syndrome, Parkinson’s disease, chronic obstructive pulmonary disease, biliary cirrhosis, hepatitis, stroke, brain injury and systemic lupus erythematosus (Fisk \& Doble, 2002). The developers of the FIS also validated the Daily Fatigue Impact Scale (D-FIS) in a sample of patients with flu-like symptoms (Fisk \& Doble, 2002). The D-FIS is applicable to capture shortterm changes in fatigue. The D-FIS was also validated in a sample of Spanish patients with Parkinson’s disease (Martinez-Martin et al., 2006). Prospective studies on the construct validity of D-FIS are yet to be conducted. 


\section{Visual Analogue Scale for Fatigue}

The Visual Analogue Scale for Fatigue (VAS-F) is an 18 item scale measuring two dimensions:

energy and fatigue (Lee, et al., 1991). The scales are $100 \mathrm{~mm}$ lines and respondents are asked to indicate their responses on a continuum ranging from not at all to extremely. The VAS-F was validated in samples of healthy adults and patients with sleep disorders (Lee, et al., 1991). The majority of items are simply adjectives synonym with fatigue or energy. The concurrent validity of the scale was established comparing it to the Stanford Sleepiness Scale. Also, at visual inspection, at least four items directly assess sleepiness. The fact that the VAS-F does not discriminate between sleepiness and fatigue limits its use in clinical practice and in research.

\section{Brief Fatigue Inventory}

The Brief Fatigue Inventory (BFI) was developed to provide a simple self-report measure for cancer patients, which is easy to understand and complete (Anderson et al., 2003). The BFI is a unidimensional scale measuring fatigue severity. The scale includes 9 items with 11-point Likert type scales. Scores 0-3 indicate mild fatigue; 4-6 suggest moderate fatigue and scores 7-10 signal severe fatigue. Respondents rate their worst, usual and current level of fatigue as well as the impact of fatigue in the past 24 hours. In one study, the BFI has differentiated among patients with mood disorders, cancer patients and community dwelling adults (Anderson, et al., 2003).

\section{Fatigue Symptom Inventory}

The Fatigue Symptom Inventory (FSI) was developed to provide a multi-dimensional assessment of cancer-related fatigue (Hann, et al., 1998). The FSI has 13 items that load into three dimensions: fatigue intensity; fatigue duration and impact of fatigue. The items consist of 11 point Likert scales, ranging from 0 (not at all fatigued/ no interference) to 10 (as fatigued as could be/extreme interference). The original validation samples were women receiving 
radiotherapy, women after breast cancer treatment and healthy women who never had cancer (Hann, et al., 1998). The convergent validity of the questionnaire was established using the Profile of Mood States Fatigue/Inertia subscale (POMS-F) and the Medical Outcomes Study Short Form -36 (SF-36) vitality subscale. This raises some concern since the fatigue/vitality subscales of general health questionnaires are not validated independently for the assessment of fatigue. The divergent validity and the test-retest reliability of the scale were not established. In a subsequent study the authors validated the FSI in male and in older cancer patients as well (Hann, Denniston, \& Baker, 2000). 


\section{Appendix B: Interview Guide}

\section{FATIGUE IN CHRONIC HEPATITIS C INFECTION}

\section{General Characteristics of Fatigue}

\section{Fatigue and/or sleepiness}

- Could you tell me whether fatigue and sleepiness are different for you? How?

- I would like to give you definition of sleepiness and fatigue:

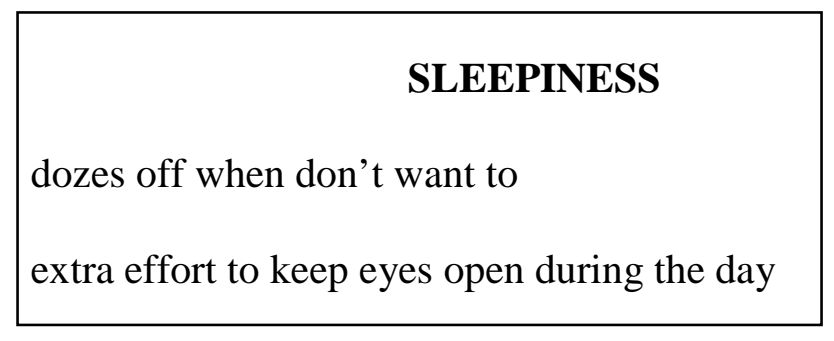

\begin{tabular}{|l|}
\hline FATIGUE \\
exhausted \\
lack of energy \\
\hline
\end{tabular}

Would you say that you tend to be sleepy or fatigued or both? Which one is the bigger problem for you? If fatigue is the main problem, then focus on fatigue in the rest of the interview. If sleepiness is the bigger problem, ask participant whether would like to talk about sleepiness first or it is OK to talk only about only fatigue. If sleepiness is an issue, discuss fatigue in a separate section and confirm definition and focus repeatedly.

\section{Description of fatigue}

- How would you describe your fatigue?

- If someone had never experienced fatigue, what would you tell it is like?

\section{Normal/pathological fatigue?}

Is your fatigue typical/normal?

- compared to your normal

- compared to other people 
If not, what makes it different from normal fatigue?

\section{Course of fatigue}

When did fatigue start to be a problem for you? How does your fatigue changes over time?

- Within a day, over days, week, year?

- Is there a pattern?

- How predictable are these changes?

\section{Functional Impact}

- What impact has fatigue had on your life?

- How does fatigue affect your everyday life?

- What are the things you give up doing when you feel tired?

- What are the things that you can still do when you are fatigued?

- How would your life be different if you were less fatigued?

\section{Fatigue Related Behaviour Factors}

- What do you do to try to prevent fatigue? How (well) do these strategies work?

- How do you cope with fatigue? What do you do when you feel tired? How do you make it better?

- How confident are you that you can manage your fatigue?

\section{Attributions and Link between Fatigue and Comorbidities}

\section{Attributions}

- How would you explain why you are fatigued?

- If we draw a circle- say it’s a fatigue pie- could you slice it so that each slice represents one thing that contributes to your fatigue? The bigger the slice, the bigger the contribution is. 


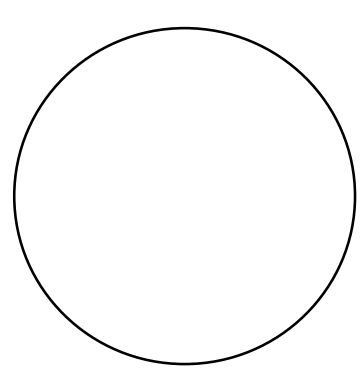

- What role do you think your hepatitis infection plays in your fatigue?

- What role do other factors play?
o Sleep
o Mood
o Stress
o Activity level

- Does fatigue affect your

o Infection

o Sleep habits

o Mood

o Stress level

o Your confidence to cope with the infection (sleep problem, mood problem, life stressors)

What role does your fatigue play in your infection/sleep habits...?

\section{Rumination about fatigue}

- How much do you think about your fatigue?

o How often?

o For how long?

o Do you find that you keep thinking about your fatigue more than you want to? 
- If I put a loudspeaker in your head, what would I hear when you think about your fatigue?

- What do you tell to yourself, when you are fatigued?

\section{Communication about Fatigue}

- To whom do you talk to about your fatigue?

- How do you decide whom to tell?

- How do these people respond?

- Do you tell your health professionals about your fatigue?

- If yes, how do you tell them?

- How do they help you?

- How confident are you that they can help you?

\section{Prognosis}

Could you predict how do you think your fatigue will be in the future?

- Next few months

- Years 


\section{Appendix C: Code Books \\ Draft Code Book A}

\section{The Trajectory of Fatigue}

A previous qualitative study on fatigue in hepatitis $C$ described 2 distinct fatigue trajectories: chronic (permanent) and idiopathic (transient, unexpected and overwhelming). For the purpose of the present study, the initial themes are chronic and transient, where transient refers only the temporal course of fatigue without its subjective (unexpected and overwhelming) quality.

Theme Description

Chronic persistent

Transient

present -absent 


\section{Draft Code Book B}

\section{Triggers and Alleviators of Fatigue}

The only evidence on the specific triggers and alleviators of fatigue in chronic hepatitis $\mathrm{C}$ is related to treatment: patients are likely experience significant fatigue when they are on combination antiviral treatment. One presumes that increased fatigue may also be triggered by the onset of depressive episodes in patients suffering from depression. Further, individuals with or without insomnia often link a burst of “tiredness” to recent poor or inadequate amount of sleep. Based on personal clinical experience, acute "stress” and "overwork” is also reported to trigger fatigue. In terms of alleviators, normal fatigue lifts after rest whereas pathological fatigue is resistant to change.

\section{Theme}

\section{Description}

Trigger: Antiviral Treatment

Fatigue starts/ increases after the initiation of antiviral treatment

Trigger: Depressive episode

Fatigue is exacerbated by the onset of a depressive episode

Trigger: Sleep

Fatigue is triggered by a period of insomnia or sleep deprivation

Trigger: Stress

Fatigue is triggered by acute stress

Alleviator: Rest

Fatigue is alleviated by rest

Alleviator: Nothing

Fatigue is resistant to common interventions 


\section{Draft Code Book C}

\section{The Functional Impact of Fatigue}

The themes related to the issue of functional impact cover the areas included in the FSS

and the Illness Intrusiveness Scale (Devins et al., 1983; Krupp, et al., 1989a).

Theme Description

Physical functioning

Work

Social Life

Family Life

Active recreation

Passive recreation

Financial Situation

Self-improvement 


\section{Draft Code Book D}

\section{Cognitive Factors- Fatigue Attributions}

The themes of the coding template for fatigue related attributions were chosen based on data from previous research in other medical conditions and on the researcher's clinical experience (Skerrett \& Moss-Morris, 2006; Wessely, David, Butler, \& Chalder, 1989).

\section{Theme Description}

Hepatitis C infection

Treatment of Hepatitis C

Other medical conditions

Inadequate sleep

Activity level

Diet

Stress

Lack of social support
Past combination treatment

Medical conditions other than hepatitis C infection, including depression and anxiety disorders. Includes treatment of other medical conditions.

Insufficient sleep quantity or quality, non-refreshing sleep, sleep disorders.

Too many/intense activity or inactivity

Overeating/irregular meals/unhealthy diet

Stress related to work, family life, finances, unemployment, health of self or others

Inadequate perceived social support, including family, friends, and health care team 


\section{Draft Code Book E}

\section{Cognitive Factors- Other}

Further fatigue-related cognitive factors described in chronic medical conditions are rumination about fatigue, catastrophizing, lack of control and beliefs about activity (Gielissen, et al., 2006; Harris \& Carney, 2012; Knoop, et al., 2010)

\section{Theme}

\section{Description}

Fatigue focused rumination

Catastrophizing

Lack of control

Negative activity 


\section{Draft Code Book F}

\section{Fatigue- Related Behavioural Factors}

Behavioural responses to the experience of fatigue and fatigue-related coping strategies belong to this category. The themes of the codebook were drawn from previous research in other populations and from the researcher's clinical experience (White, et al., 2011)

\begin{tabular}{ll}
\hline \multicolumn{1}{c}{ Theme } & \multicolumn{1}{c}{ Description } \\
\hline Rest & Sedentary activities but not sleep, stay in bed more than usual \\
Sleep & Sleep more than usual \\
“Take a break” & Vacation, days off \\
Exercise & Vigorous physical activity \\
Active recreation & Rewarding, pleasurable activities \\
Pacing & Ask others to take over tasks \\
Delegate tasks & Beverages with caffeine content, chocolate, psycho-stimulant \\
Stimulants & drugs
\end{tabular}




\title{
Final Code books A, B, C: The general characteristics of fatigue
}

\author{
Final Code book A
}

Characteristics of Fatigue

Relevant interview section: 1.3

\begin{tabular}{|c|c|c|}
\hline Theme & Definition & $\begin{array}{l}\text { Instructions for } \\
\text { Coding }\end{array}$ \\
\hline Starts earlier than normal & $\begin{array}{l}\text { Fatigue starts earlier than } \\
\text { participants think it is normal }\end{array}$ & \\
\hline Persistent & $\begin{array}{l}\text { Fatigue is present persistently } \\
\text { (more days then not/ lasts long) }\end{array}$ & \\
\hline Physical & $\begin{array}{l}\text { Fatigue is described as a } \\
\text { physical experience (e.g. } \\
\text { muscle, body, physical fatigue) }\end{array}$ & \\
\hline Cognitive & $\begin{array}{l}\text { Fatigue is described as a } \\
\text { cognitive experience (e.g. } \\
\text { brain, focus, concentration) }\end{array}$ & \\
\hline Emotions as a consequence & Emotions related to fatigue & $\begin{array}{l}\text { Do not code illness } \\
\text { related anxiety here if it } \\
\text { exacerbates fatigue }\end{array}$ \\
\hline
\end{tabular}




\section{Final Code Book B}

\section{The course of fatigue}

Relevant interview sections: 1.4

Theme

Chronic stable

Chronic progressive
Problematic fatigue has been present for months/years. The subjective severity has not changed over time.

Description

Problematic fatigue has been present for months/years. It has become progressively worse over time. 


\section{Final Code Book C}

\section{Triggers of fatigue}

Relevant interview section: triggers and alleviators of fatigue

\begin{tabular}{|c|c|c|}
\hline Theme & Description & $\begin{array}{l}\text { Instructions } \\
\text { for Coding }\end{array}$ \\
\hline Activity & $\begin{array}{l}\text { Fatigue is exacerbated by } \\
\text { physical activity (including } \\
\text { physical work, chores, exercise, } \\
\text { walking, etc.) or mental activity, } \\
\text { or sustained activity }\end{array}$ & \\
\hline Stress & $\begin{array}{l}\text { Fatigue is exacerbated by stress } \\
\text { related to any life area (e.g. } \\
\text { family, work, health, finances, } \\
\text { etc.) }\end{array}$ & $\begin{array}{l}\text { Code when acute stress due to } \\
\text { momentarily life events exacerbate } \\
\text { fatigue (e.g. acute conflict, or life } \\
\text { event at home or at work) }\end{array}$ \\
\hline $\begin{array}{l}\text { Illness related } \\
\text { anxiety/uncertainty }\end{array}$ & $\begin{array}{l}\text { Fatigue is related to } \\
\text { anxiety/uncertainty about one's } \\
\text { health and the prognosis of the } \\
\text { liver disease }\end{array}$ & \\
\hline Sleep & Sleep exacerbates fatigue & \\
\hline Antiviral treatment & $\begin{array}{l}\text { Antiviral treatment increased } \\
\text { fatigue }\end{array}$ & \\
\hline
\end{tabular}




\section{Final Code Book E}

\section{The Functional Impact of Fatigue}

\begin{tabular}{|c|c|c|}
\hline Theme & Definition & Instructions for coding \\
\hline Physical functioning & $\begin{array}{l}\text { Fatigue impacts one's ability to engage in routine } \\
\text { physical activities (including physical work, chores, } \\
\text { walking) or impaired performance related to physical } \\
\text { activities (can do less, takes longer to complete, } \\
\text { slower etc.) due to fatigue. }\end{array}$ & $\begin{array}{l}\text { Do not code if physical activity takes place in the } \\
\text { context of family life, social life, work or active } \\
\text { recreation. For example, DO NOT code exercise } \\
\text { here, or skating with grandchildren here. Exercise } \\
\text { is coded under active recreation, skating with } \\
\text { grandkids is coded under family life. }\end{array}$ \\
\hline Work & $\begin{array}{l}\text { Fatigue causes impaired work performance, } \\
\text { decreased satisfaction with work, absenteeism, sick } \\
\text { leave, inability to volunteer }\end{array}$ & \\
\hline Social Life & $\begin{array}{l}\text { Fatigue has a negative impact on social life, } \\
\text { including type and frequency of social activities that } \\
\text { one engages in. }\end{array}$ & $\begin{array}{l}\text { Do not code social activities with family } \\
\text { members here, for example going to the movie } \\
\text { with one's children. Code those under family life. } \\
\text { Do not code recreation with friends here, code } \\
\text { those under social activity. }\end{array}$ \\
\hline $\begin{array}{l}\text { Family Life and Intimate } \\
\text { Relationships }\end{array}$ & $\begin{array}{l}\text { Fatigue has a negative impact on relationship with } \\
\text { family members, on activities that the family engages } \\
\text { in (including recreational and social activities), roles } \\
\text { in the family, family dynamics, relationship status, } \\
\text { sexual life }\end{array}$ & \\
\hline Active recreation & $\begin{array}{l}\text { Fatigue interferes with one's ability to engage in } \\
\text { recreational activities that require physical } \\
\text { activity/active involvement e.g. sports, clubs, } \\
\text { gardening, travelling }\end{array}$ & $\begin{array}{l}\text { Do not include activities that the family engages } \\
\text { in; code those under family life. }\end{array}$ \\
\hline Finances & $\begin{array}{l}\text { Fatigue has negative impact on one's ability to } \\
\text { generate income, or to do important finance related } \\
\text { activities, for example bills, or treatment of fatigue is } \\
\text { expensive }\end{array}$ & \\
\hline Future plans & $\begin{array}{l}\text { Participant reports that he/she cannot make short or } \\
\text { longer term plans regarding activities, social life, } \\
\text { self-improvement, career, family life, finances, ways } \\
\text { of living, relationships, housing etc. because of } \\
\text { fatigue or fatigue related uncertainty }\end{array}$ & \\
\hline
\end{tabular}


Final Code Book F

Cognitive factors- Fatigue Attributions

Relevant interview sections: 4

Theme

Description

Hepatitis C infection

Hepatitis C infection causes fatigue

Other medical conditions

Medical conditions other than hepatitis C infection, including depression and anxiety disorders

contribute to fatigue. Includes treatment of other medical conditions.

Sleep disorders/chronic sleep problem

Participant states that a sleep disorder or a chronic sleep problem causes the fatigue. If the participant reports that she/he feels more tired after a bad night sleep, but it happens only occasionally, code as a trigger. 


\section{Final Code Book G}

\section{Cognitive factors- other}

Relevant interview sections: 5

\begin{tabular}{ll}
\hline \multicolumn{1}{c}{ Theme } & \multicolumn{1}{c}{ Description } \\
\hline Cognitive avoidance & $\begin{array}{l}\text { Tries to avoid thinking about fatigue; keeps oneself busy in } \\
\text { order to avoid thinking about fatigue }\end{array}$ \\
Bontrol & The participant accepts his/her fatigue, comes to peace with it \\
Acceptance & $\begin{array}{l}\text { The prediction participants make about the future course of } \\
\text { their fatigue; their prediction of change over time }\end{array}$ \\
Prognosis & $\begin{array}{l}\text { A belief that positive thinking, positive attitude helps to } \\
\text { manage fatigue or to live one’s life with chronic fatigue }\end{array}$ \\
Positive attitude &
\end{tabular}




\section{Final Code Book H}

\section{Fatigue- Related Behavioural Factors}

\begin{tabular}{|c|c|c|}
\hline Theme & Description & Instruction for Coding \\
\hline Non-active rest & $\begin{array}{l}\text { Drops everything and rests but does not sleep; no activity } \\
\text { other than rest; passive without paying attention/without being } \\
\text { engaged (for example TV is on but does not pay attention; } \\
\text { book in hand but is not reading) }\end{array}$ & \\
\hline Active rest & $\begin{array}{l}\text { Physical rest but engages in goal-oriented non-physical } \\
\text { activities. Does not sleep. }\end{array}$ & $\begin{array}{l}\text { For example sits down to rest } \\
\text { physically because feels fatigued } \\
\text { but reads, organizes paperwork, } \\
\text { eats a good meal, watches favourite } \\
\text { TV show }\end{array}$ \\
\hline Sleep & Takes a nap, sleeps in, goes to sleep earlier when fatigued & \\
\hline Physical activity & $\begin{array}{l}\text { Goes for a walk, exercises etc. when fatigued or to prevent } \\
\text { fatigue }\end{array}$ & \\
\hline $\begin{array}{l}\text { Fatigue dictated } \\
\text { activity pattern }\end{array}$ & $\begin{array}{l}\text { Decides on activity based on current fatigue level; cancels } \\
\text { programs when tired; does only the bare minimum and rests }\end{array}$ & \\
\hline Pacing & $\begin{array}{l}\text { Makes activity plans in order to avoid overexertion; uses } \\
\text { energy budget to keep balance; brakes up activities into small } \\
\text { parts and schedules rest between parts; adapts intensity of the } \\
\text { activity to level of fatigue (slows down or stops when starts to } \\
\text { feel tired) in order to avoid over-exertion }\end{array}$ & \\
\hline Nutrition & $\begin{array}{l}\text { Eating healthy, special diet in order to manage fatigue/ } \\
\text { drinking beverages to manage fatigue }\end{array}$ & $\begin{array}{l}\text { Code only if participant keeps diet } \\
\text { to manage fatigue; Do not code, if } \\
\text { participant has a healthy diet to } \\
\text { maintain general health }\end{array}$ \\
\hline $\begin{array}{l}\text { Pleasurable } \\
\text { activities }\end{array}$ & Participant engages in pleasurable activities to combat fatigue & $\begin{array}{l}\text { Code only if the participant reports } \\
\text { that the activity brings joy }\end{array}$ \\
\hline
\end{tabular}




\section{Final Code Book I}

\section{Communication about fatigue}

Relevant interview section: 6

\section{Theme}

Definition
Reasons for not communicating with health Participant's explanation/reasons of why care providers not communicates about fatigue to physicians, family, friends

Communication about fatigue with health care providers

Participant talks about fatigue to family doctor. Includes what the participants say to doctors and how they respond. Participant talks about fatigue to gastroenterologist or other medical specialist. Includes what the participants say to the specialist and how they respond 


\section{Appendix D: Examples for Sleep and Fatigue Screening}

How concerned are you about your fatigue?

$\begin{array}{lrlllllllll}0 & 1 & 2 & 3 & 4 & 5 & 6 & 7 & 8 & 9 & \begin{array}{c}10 \\ \text { extremely concerned }\end{array} \\ \text { not at all } & & & & & & & & & \end{array}$

How dissatisfied are you with the quality or with the amount of your sleep?

$\begin{array}{lrlllllllll}0 & 1 & 2 & 3 & 4 & 5 & 6 & 7 & 8 & 9 & \begin{array}{l}10 \\ \text { extremely dissatisfied }\end{array} \\ \text { not at all } & & & & & & & & \end{array}$

\section{STOP BANG}

Do you Snore?

Do you feel Tired, fatigued or sleepy during the day?

Has anyone Observed you stop breathing in your sleep?

Do you have high blood Pressure?

Yes_ ${ }^{\mathrm{No}}-$
Yes_ ${ }^{\mathrm{No}}-$
Yes_ No -
Yes__No_-

Are you a male?

Yes_ No _

Are you 50 or older?

Yes _ No _

Is your neck size more than $40 \mathrm{~cm} / 16$ "?

Yes _ No _

Please circle below your weight and height EITHER in $\mathrm{ft}$ and $\mathrm{lbs} \mathrm{OR}$ in $\mathrm{cm}$ and $\mathrm{kg}$. If your weight or height is below these values, leave it blank.

If you know your weight and height in $\underline{\mathbf{f t} \text { and } \mathbf{l b s}}$ please circle here:

How tall are you? 4'10" 5'0" 5'2" 5'4" 5'6" 5'6" 5'10" 6'0" 6'2" 6'4" ft. in.

$\begin{array}{llllllllllll}\text { What is your weight? } & 167 & 179 & 191 & 204 & 216 & 230 & 250 & 258 & 272 & 287 \mathbf{l b} .\end{array}$

If you know your weight and height in $\underline{\mathbf{c m}}$ and $\mathbf{~ k g}$ please circle here:

$\begin{array}{llllllllllll}\text { How tall are you? } & 147 & 152 & 158 & 163 & 168 & 173 & 178 & 183 & 188 & 193 & \text { cm }\end{array}$

$\begin{array}{lllllllllll}\text { What is your weight? } & 75 & 81 & 86 & 92 & 97 & 104 & 113 & 116 & 122 & 129 \\ \mathbf{~ k g}\end{array}$ 
An Example for Fatigue and Sleep Referral Algorithm for Patients with Hepatitis C Infection Note: Medical comorbidities must be assessed and treated first

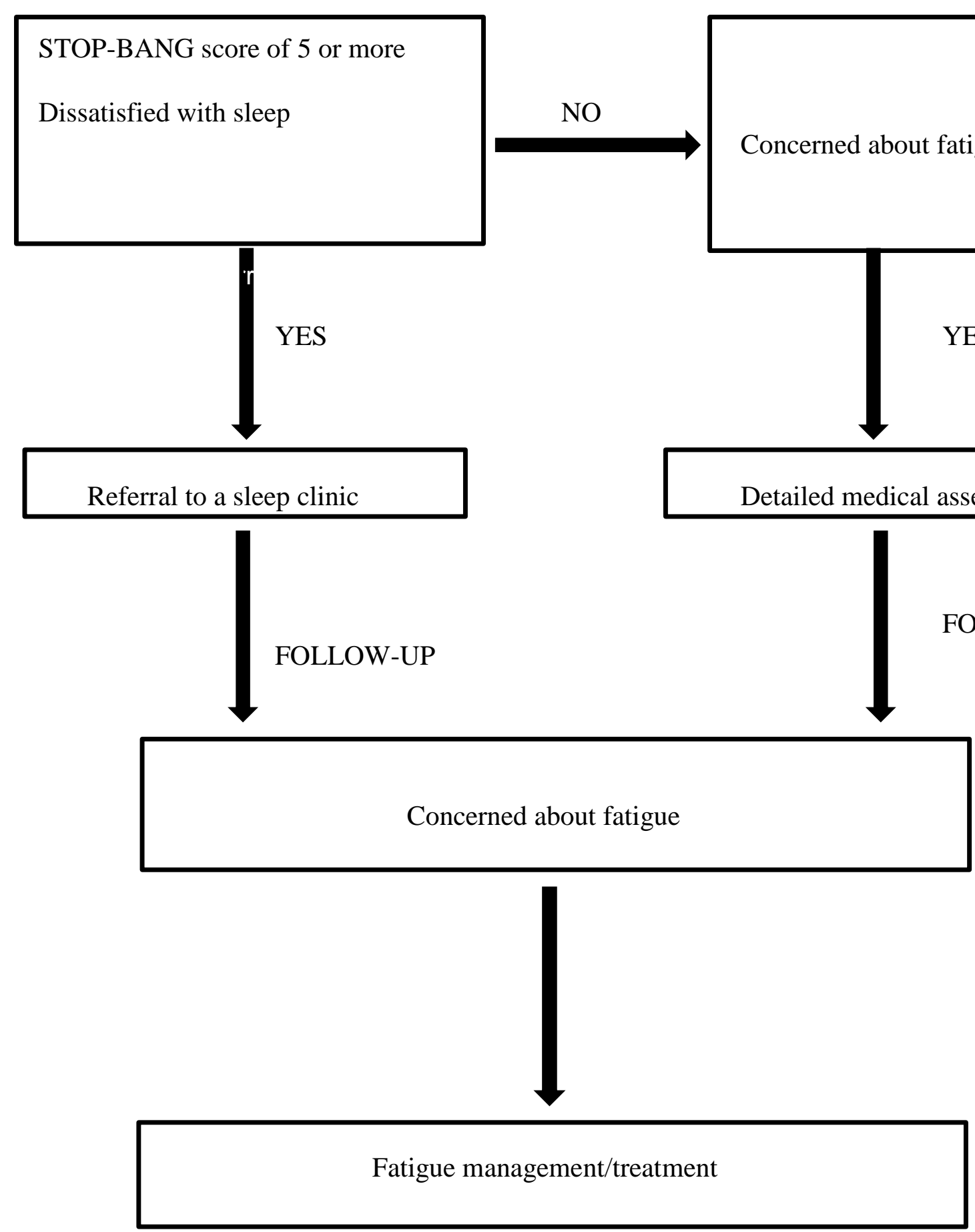




\section{Appendix E: The Fatigue Perception Model}

A simple, cognitive model of fatigue in the context of chronic medical conditions is presented below.

Insert Figure 11 here

This model builds on a previous model of fatigue in neurological conditions and on Leventhal's self- regulation model of illness perception (Chaudhuri \& Behan, 2004; Levethal, 1984). Similar to a previous model of central fatigue, fatigue perception model posits that experienced fatigue in the medical conditions assumes physiological changes characterizing the illness, secondary physiological processes in the neuromuscular and in the central nervous system and the detection of these on the cortical level. These processes are described and depicted in more detail in the neurological model of fatigue on page 5 and in (Chaudhuri \& Behan, 2004). The fatigue perception model suggests that the experience of fatigue arises from the combination of these physiological, neurological and more abstract mental processes. The latter refers to mental fatigue representations/ "perceptions”/cognitions. These cognitive entities represent the individual's knowledge and beliefs of fatigue formed from previous, own fatigue experiences, vicarious learning, cultural learning etc. There are also culturally shared beliefs about fatigue that an individual may endorse (Karasz \& McKinley, 2007). The persons’ cognitions about fatigue influence whether one engages in fatigue management behaviours and what behaviours one choses to perform. For example, the general experience of fatigue that it is temporary. When fatigue is a temporary experience one can chose to "press on" since fatigue is normal and will dissipate eventually anyway. We also learn from childhood that we may need to 
stay at home and need to rest when we have a fever and that we regain our energy in few days. Riding on a temporary wave of fatigue or resting are adaptive behaviours to manage a common phenomenon, namely temporary fatigue.

When one is diagnosed with a medical condition, an additional explanation for fatigue becomes available viz. a symptom of the illness. As discussed in the present study, beliefs about fatigue may evoke negative emotions, if the illness that the fatigue is associated with is threatening or if fatigue has perceived severe negative consequences on one's performance in various social roles. Beliefs about fatigue also guide coping behaviours. Someone who attributes daytime fatigue to an episode of insomnia may attempt to obtain more sleep in the hope of controlling fatigue. Another person may think that fatigue is a sign of accumulation of the toxins in the body that a sick kidney or liver could not clear. This person may start a special diet to combat fatigue, as some participants in the current sample have done. According the Leventhal's model (1984), the cognition-behaviour-emotion system is dynamic, in that the outcome of coping behaviours changes cognitions that lead to behavioural adjustment. Coping behaviours that provide short-term benefit can be reinforced, even though they may maintain chronic fatigue. For example, restricting activities may result in: physiological changes (e.g. deconditioning, disruption of circadian rhythm); emotional reactions (e.g. sadness, boredom); and neuropsychological changes (e.g. attention to physical sensations) all of which will shape the experience of fatigue.

An important implication of the model is that illness and fatigue are related not just on the physiological, but also on the cognitive, behavioural, and emotional levels. Fatigue-specific cognitions and fatigue management behavioural repertoire are present independently of the illness but the individuals' beliefs about the illness, their illness related emotions and illness 
management behaviours may influence their fatigue-specific beliefs, emotions, and behaviours. The differences in the physiological, cognitive, emotional and behavioural context of individual medical conditions may help to explain why fatigue can be different in different medical conditions. The examples of fatigue focused rumination and acceptance illustrate this point.

Rumination is a repetitive, self-focused, retrospective thinking process. In the present study participants with high fatigue and insomnia ruminated significantly more about their fatigue than those with high fatigue but without insomnia. Individuals with insomnia tend to attribute their fatigue to their poor sleep (Harris \& Carney, 2012). They also notice that they sleep more on some nights than on others. Rumination about fatigue "makes sense" in the context of insomnia, since the factors underlying the variability of sleep might explain why one feels more tired on some days than on others. Rumination in those with insomnia may therefore be a problem solving strategy (Carney, et al., 2013). In contrast, rumination about fatigue in the context of HCV infection is probably a less plausible fatigue management strategy, since the infection has a long term course and there are no daily fluctuations in the disease severity. Indeed, a number of participants in the current study insisted that that they did not spend a lot of time thinking about their fatigue, they rather accepted to have a chronic fatigue.

An interesting difference between the present sample and published data on a sample with chronic fatigue syndrome is that fatigue acceptance was higher in this group than it was in the chronic fatigue group. Qualitative research, including the current study, shows that patients with chronic medical conditions may believe that fatigue is an integral, inevitable part of their disease (Kralik, Telford, Price, \& Koch, 2005; Repping-Wuts, et al., 2008). Acceptance appears to be a logical coping behaviour in these conditions. Conversely, in chronic fatigue syndrome, which is a disorder of unknown origin that may cause a dramatic change in the productivity of 
often perfectionist individuals, continuous attempts to control fatigue and to maintain one's usual performance level may be a more typical coping strategy.

Ultimately, similarities are likely to exceed the differences in fatigue cognitions and behaviours across medical conditions. Also, individual differences can be substantial and are important to recognize in tailor made, psychological interventions. Nevertheless, the model may have utility for future research on the interaction between illness and fatigue-specific cognitions and behaviours. It also may be adapted for use in patient education on fatigue to highlight the pathways through which physiological and psychological processes interact to give rise to the unique experience of fatigue. 


\section{References}

Aguillard, R. N., Riedel, B. W., Lichstein, K. L., Grieve, F. G., Johnson, C. T., \& Noe, S. L. (1998). Daytime functioning in obstructive sleep apnea patients: exercise tolerance, subjective fatigue, and sleepiness. Applied Psychophysiology and Biofeedback, 23(4), 207-217.

Amtmann, D., Bamer, A. M., Noonan, V., Lang, N., Kim, J., \& Cook, K. F. (2012). Comparison of the psychometric properties of two fatigue scales in multiple sclerosis. Rehabilitation Psychology, 57(2), 159-166.

Anderson, Getto, C. J., Mendoza, T. R., Palmer, S. N., Wang, X. S., Reyes-Gibby, C. C., \& Cleeland, C. S. (2003). Fatigue and sleep disturbance in patients with cancer, patients with clinical depression, and community-dwelling adults. Journal of Pain and Symptom Management, 25(4), 307-318.

Anderson, Jason, L. A., Hlavaty, L. E., Porter, N., \& Cudia, J. (2012). A review and metasynthesis of qualitative studies on myalgic encephalomyelitis/chronic fatigue syndrome. Patient Education and Counseling, 86(2), 147-155.

Antony, M. M., Bieling, P. J., Cox, B. J., Enns, M. W., \& Swinson, R. P. (1998). Psychometric properties of the 42 -item and 21-item versions of the Depression Anxiety Stress Scales in clinical groups and a community sample. Psychological Assessment, 10, 176-181.

Armes, J., Chalder, T., Addington-Hall, J., Richardson, A., \& Hotopf, M. (2007). A randomized controlled trial to evaluate the effectiveness of a brief, behaviorally oriented intervention for cancer-related fatigue. Cancer, 110(6), 1385-1395.

Bailey, D. E., Jr., Barroso, J., Muir, A. J., Sloane, R., Richmond, J., McHutchison, J., ... Mishel, M. H. (2010). Patients with chronic hepatitis C undergoing watchful waiting: Exploring 
trajectories of illness uncertainty and fatigue. Research in Nursing Health, 33(5), 465473.

Bastien, C. H., Vallieres, A., \& Morin, C. M. (2001). Validation of the Insomnia Severity Index as an outcome measure for insomnia research. Sleep Medicine, 2, 297-307.

Bedossa, P. (2008). Liver biopsy. Gastroenterologie clinique et biologique, 32(6 Suppl 1), 4-7.

Bensing, J. M., \& Verhaak, P. F. M. (2004). Communication in Medical Encounters. In A. Kaptein \& J. Weinman (Eds.), Health Psychology (pp. 261-287). Oxford, UK: The British Psychological Association.

Bieling, P. J., Antony, M. M., \& Swinson, R. P. (1998). The State-Trait Anxiety Inventory, Trait version: structure and content re-examined. Behav Res Ther, 36(7-8), 777-788.

Bol, Y., Duits, A. A., Hupperts, R. M., Vlaeyen, J. W., \& Verhey, F. R. (2009). The psychology of fatigue in patients with multiple sclerosis: a review. Journal of Psychosomatic Research, 66(1), 3-11.

Bol, Y., Duits, A. A., Lousberg, R., Hupperts, R. M., Lacroix, M. H., Verhey, F. R., \& Vlaeyen, J. W. (2010). Fatigue and physical disability in patients with multiple sclerosis: a structural equation modeling approach. Journal of Behavioral Medicine, 33(5), 355-363.

Broadbent, E., Ellis, C. J., Thomas, J., Gamble, G., \& Petrie, K. J. (2009). Further development of an illness perception intervention for myocardial infarction patients: a randomized controlled trial. Journal of Psychosomatic Research, 67(1), 17-23.

Broadbent, E., Petrie, K. J., Main, J., \& Weinman, J. (2006). The brief illness perception questionnaire. Journal of Psychosomatic Research, 60(6), 631-637.

Brooks, S. K., Rimes, K. A., \& Chalder, T. (2011a). The role of acceptance in chronic fatigue syndrome. Journal of Psychosomatic Research 71(6), 411-415. 
Brooks, S. K., Rimes, K. A., \& Chalder, T. (2011b). The role of acceptance in chronic fatigue syndrome. Journal of Psychosomatic Research, 71(6), 411-415.

Brown, L. F., \& Kroenke, K. (2009). Cancer-related fatigue and its associations with depression and anxiety: a systematic review. Psychosomatics, 50(5), 440-447.

Budhiraja, R., Roth, T., Hudgel, D. W., Budhiraja, P., \& Drake, C. L. (2011). Prevalence and polysomnographic correlates of insomnia comorbid with medical disorders. Sleep, 34(7), 859-867.

Buysse, D. J., Ancoli-lsrael, S., Edinger, J. D., Lichstein, K. L., \& Morin, C. M. (2006).

Recommendations for a standard research assessment of insomnia. Sleep: Journal of Sleep and Sleep Disorders Research, 29(9), 1155-1173.

Buysse, D. J., Thompson, W., Scott, J., Franzen, P. L., Germain, A., Hall, M., . . Kupfer, D. J. (2007). Daytime symptoms in primary insomnia: a prospective analysis using ecological momentary assessment. Sleep Medicine, 8(3), 198-208.

Campbell-Sills, L., \& Brown, A. (2011). Generalized Anxiety Disorder. In M. M. Antony \& H. B. Barlow (Eds.), Handbook of Assessment and Treatment Planning for Paychological Disorders (Second ed., pp. 224-266). New York, NY: The Guilford Press.

Canada, P. H. A. o. (2011). Hepatitis C in Canada: 2005-2010 Surveillance Report. In P. H. A. o. C. Centre for Communicable Diseases and Infection Control (Ed.): Public Health Agency of Canada.

Carlson, M. D., Hilsabeck, R. C., Barakat, F., \& Perry, W. (2010). Role of Sleep Disturbance in Chronic Hepatitis C Infection. Current Hepatitis Reports, 9(1), 25-29. 
Carney, Harris, A. L., Falco, A., \& Edinger, J. D. (2013). The Relation between Insomnia Symptoms, Mood and Rumination about Insomnia Symptoms. Journal of Clinical Sleep Medicine, 9, 567-575.

Castera, L., Forns, X., \& Alberti, A. (2008). Non-invasive evaluation of liver fibrosis using transient elastography. Journal of Hepatology, 48(5), 835-847.

Chalder, T., Berelowitz, G., Pawlikowska, T., Watts, L., Wessely, S., Wright, D., \& Wallace, E. P. (1993). Development of a fatigue scale. Journal of Psychosomatic Research, 37(2), $147-153$.

Chaudhuri, A., \& Behan, P. O. (2000). Fatigue and basal ganglia. Journal of the Neurological Sciences, 179(S 1-2), 34-42.

Chaudhuri, A., \& Behan, P. O. (2004). Fatigue in neurological disorders. Lancet, 363(9413), 978-988.

Choo, Q. L., Kuo, G., Weiner, A. J., Overby, L. R., Bradley, D. W., \& Houghton, M. (1989). Isolation of a cDNA clone derived from a blood-borne non-A, non-B viral hepatitis genome. Science, 244(4902), 359-362.

Chung, F., Subramanyam, R., Liao, P., Sasaki, E., Shapiro, C., \& Sun, Y. (2012). High STOPBang score indicates a high probability of obstructive sleep apnoea. British Journal of Anaesthesia, 108(5), 768-775.

Chung, F., Yegneswaran, B., Liao, P., Chung, S. A., Vairavanathan, S., Islam, S., ... Shapiro, C. M. (2008). STOP questionnaire: a tool to screen patients for obstructive sleep apnea. Anesthesiology, 108(5), 812-821. 
Ciftci, T. U., Kokturk, O., Bukan, N., \& Bilgihan, A. (2004). The relationship between serum cytokine levels with obesity and obstructive sleep apnea syndrome. Cytokine, 28(2), 8791.

Cohen, J. (1992). A power primer. Psychological Bulletin, 112(1), 155-159.

Colloca, L., \& Miller, F. G. (2011). The nocebo effect and its relevance for clinical practice. [Review]. Psychosomatic Medicine, 73(7), 598-603.

Crow, R., Gage, H., Hampson, S., Hart, J., Kimber, A., \& Thomas, H. (1999). The role of expectancies in the placebo effect and their use in the delivery of health care: a systematic review. Health Technology Assessment, 3(3), 1-96.

DeLuca, J. (2005). Fatigue as a window to the brain. Massachusett: MIT Press.

Devins, G. M., Binik, Y. M., Hutchinson, T. A., Hollomby, D. J., Barre, P. E., \& Guttmann, R. D. (1983). The emotional impact of end-stage renal disease: importance of patients' perception of intrusiveness and control. International Journal of Psychiatry in Medicine, 13(4), 327-343.

Dimsdale, J. E., Ancoli-Israel, S., Ayalon, L., Elsmore, T. F., \& Gruen, W. (2007). Taking fatigue seriously, II: variability in fatigue levels in cancer patients. Psychosomatics, 48(3), 247-252.

Dittner, A. J., Wessely, S. C., \& Brown, R. G. (2004). The assessment of fatigue: a practical guide for clinicians and researchers. Journal of Psychosomatic Research, 56(2), 157-170.

Dwight, M. M., Kowdley, K. V., Russo, J. E., Ciechanowski, P. S., Larson, A. M., \& Katon, W. J. (2000). Depression, fatigue, and functional disability in patients with chronic hepatitis C. Journal of Psychosomatic Research, 49(5), 311-317. 
Elbers, R. G., Rietberg, M. B., van Wegen, E. E., Verhoef, J., Kramer, S. F., Terwee, C. B., \& Kwakkel, G. (2012). Self-report fatigue questionnaires in multiple sclerosis, Parkinson's disease and stroke: a systematic review of measurement properties. Quality of Life Research, 21(6), 925-944.

Elbers, R. G., van Wegen, E. E., Verhoef, J., \& Kwakkel, G. (2012). Reliability and structural validity of the Multidimensional Fatigue Inventory (MFI) in patients with idiopathic Parkinson's disease. Parkinsonism \& Related Disorders, 18(5), 532-536.

Ettinger, A. B., Weisbrot, D. M., Krupp, L., Coyle, P. K., Jandorf, L., \& Devinsky, O. (1998). Fatigue and Depression in Epilepsy. Journal of Epilepsy, 11, 105-109.

Ferrando, S., Evans, S., Goggin, K., Sewell, M., Fishman, B., \& Rabkin, J. (1998). Fatigue in HIV illness: relationship to depression, physical limitations, and disability. Psychosomatic Medicine, 60(6), 759-764.

Field, A. (2009). Discovering Statistics Using SPSS (Third ed.). London: Sage Publications Ltd. Fillion, L., Gelinas, C., Simard, S., Savard, J., \& Gagnon, P. (2003). Validation evidence for the French Canadian adaptation of the Multidimensional Fatigue Inventory as a measure of cancer-related fatigue. Cancer Nursing, 26(2), 143-154.

Fisk, J. D., \& Doble, S. E. (2002). Construction and validation of a fatigue impact scale for daily administration (D-FIS). Quality of Life Research, 11(3), 263-272.

Fisk, J. D., Ritvo, P. G., Ross, L., Haase, D. A., Marrie, T. J., \& Schlech, W. F. (1994). Measuring the functional impact of fatigue: initial validation of the fatigue impact scale. Clinical Infectious Diseases, 18 Suppl 1, S79-83. 
Foster, G. R., Goldin, R. D., \& Thomas, H. C. (1998). Chronic hepatitis C virus infection causes a significant reduction in quality of life in the absence of cirrhosis. Hepatology, 27(1), 209-212.

Friedman, J. H., Alves, G., Hagell, P., Marinus, J., Marsh, L., Martinez-Martin, P., . . Schrag, A. (2010). Fatigue rating scales critique and recommendations by the Movement Disorders Society task force on rating scales for Parkinson's disease. Movement Disorders, 25(7), 805-822.

Friedrich-Rust, M., Ong, M. F., Martens, S., Sarrazin, C., Bojunga, J., Zeuzem, S., \& Herrmann, E. (2008). Performance of transient elastography for the staging of liver fibrosis: a metaanalysis. Gastroenterology, 134(4), 960-974.

Galer, B. S., Schwartz, L., \& Turner, J. A. (1997). Do patient and physician expectations predict response to pain-relieving procedures? The Clinical Journal of Pain, 13(4), 348-351.

Gandevia, S. C. (2001). Spinal and supraspinal factors in human muscle fatigue. Physiological Reviews, 81(4), 1725-1789.

Gentile, S., Delaroziere, J. C., Favre, F., Sambuc, R., \& San Marco, J. L. (2003). Validation of the French 'multidimensional fatigue inventory' (MFI 20). European Journal of Cancer Care (Engl), 12(1), 58-64.

Gielissen, M. F., Verhagen, S., Witjes, F., \& Bleijenberg, G. (2006). Effects of cognitive behavior therapy in severely fatigued disease-free cancer patients compared with patients waiting for cognitive behavior therapy: a randomized controlled trial. Journal of Clinical Oncology, 24(30), 4882-4887.

Glacken, M., Coates, V., Kernohan, G., \& Hegarty, J. (2003). The experience of fatigue for people living with hepatitis C. Journal of Clinical Nursing, 12(2), 244-252. 
Glacken, M., Kernohan, G., \& Coates, V. (2001). Diagnosed with Hepatitis C: a descriptive exploratory study. International Journal of Nursing Studies, 38(1), 107-116.

Goedendorp, M. M., Gielissen, M. F., Peters, M. E., Verhagen, C. A., \& Bleijenberg, G. (2011). Moderators and long-term effectiveness of cognitive behaviour therapy for fatigue during cancer treatment. Psychooncology.

Goh, J., Coughlan, B., Quinn, J., O'Keane, J. C., \& Crowe, J. (1999). Fatigue does not correlate with the degree of hepatitis or the presence of autoimmune disorders in chronic hepatitis C infection. European Journal of Gastroenterology \& Hepatology, 11(8), 833-838.

Gravitz, L. (2011). Introduction: a smouldering public-health crisis. Nature, 474(7350), S2-4.

Green, S. B. (1991). How many subjects does it take to do a regression analysis? . Multivariate Behavioural Research, 26, 499-510.

Hann, D. M., Denniston, M. M., \& Baker, F. (2000). Measurement of fatigue in cancer patients: further validation of the Fatigue Symptom Inventory. Quality of Life Research, 9(7), 847854.

Hann, D. M., Jacobsen, P. B., Azzarello, L. M., Martin, S. C., Curran, S. L., Fields, K. K., . . . Lyman, G. (1998). Measurement of fatigue in cancer patients: development and validation of the Fatigue Symptom Inventory. Quality of Life Research, 7(4), 301-310.

Harris, A. L., \& Carney, C. E. (2009). Are people with insomnia afraid of fatigue? Paper presented at the Association of Behavioral and Cognitive Therapies, New York, NY.

Harris, A. L., \& Carney, C. E. (2012). Can we modify maladaptive attributions for fatigue? Cognitive Behaviour Therapy, 41(1), 40-50.

Harvey, A. G. (2002). A cognitive model of insomnia. Behaviour Research and Therapy, 40(8), 869-893. 
Hayes, A. F. (2012). PROCESS: A versatile computational tool for observed variable mediation, moderation, and conditional process modeling. Retrieved from www.afhayes.com/public/process2012.pdf website:

Henry, J. D., \& Crawford, J. R. (2005). The short-form version of the Depression Anxiety Stress Scales (DASS-21): construct validity and normative data in a large non-clinical sample. The British Journal of Clinical Psychology, 44(Pt 2), 227-239.

Herlofson, K., \& Larsen, J. P. (2002). Measuring fatigue in patients with Parkinson's disease the Fatigue Severity Scale. European Journal of Neurology, 9(6), 595-600.

Hilsabeck, R. C., \& Malek-Ahmadi, P. (2004). Neurobehavioral correlates of chronic hepatis C infection. Journal of Psychopathology and Behavioral Assessment, 26(3), 203-210.

Hotamisligil, G. S. (2006). Inflammation and metabolic disorders. Nature, 444(7121), 860-867.

Irwin, M. R., \& Miller, A. H. (2007). Depressive disorders and immunity: 20 years of progress and discovery. Brain, Behavior, and Immunity, 21(4), 374-383.

Jackson, M. L., \& Bruck, D. (2012). Sleep abnormalities in chronic fatigue syndrome/myalgic encephalomyelitis: a review. Journal of Clinical Sleep Medicine, 8(6), 719-728.

Jacobsen, P. B., Donovan, K. A., Vadaparampil, S. T., \& Small, B. J. (2007). Systematic review and meta-analysis of psychological and activity-based interventions for cancer-related fatigue. Health Psychology, 26(6), 660-667.

Johns, M. W. (1991). A new method for measuring daytime sleepiness: the Epworth sleepiness scale. Sleep, 14(6), 540-545.

Johnson, M. E., Fisher, D. G., Fenaughty, A., \& Theno, S. A. (1998). Hepatitis C virus and depression in drug users. The American Journal of Gastroenterology, 93(5), 785-789. 
Jopson, N. M., \& Moss-Morris, R. (2003). The role of illness severity and illness representations in adjusting to multiple sclerosis. Journal of Psychosomatic Research, 54(6), 503-511; discussion 513-504.

Kallman, J., O'Neil, M. M., Larive, B., Boparai, N., Calabrese, L., \& Younossi, Z. M. (2007). Fatigue and health-related quality of life (HRQL) in chronic hepatitis C virus infection. Digestive Diseases and Sciences, 52(10), 2531-2539.

Karasz, A., \& McKinley, P. S. (2007). Cultural differences in conceptual models of everyday fatigue: a vignette study. Journal of Health Psychology, 12(4), 613-626.

Karsdorp, P. A., \& Vlaeyen, J. W. (2009). Active avoidance but not activity pacing is associated with disability in fibromyalgia. [Research Support, Non-U.S. Gov't]. Pain, 147(1-3), 2935. doi: 10.1016/j.pain.2009.07.019

Kelly, S. E. (2013). Qualitative Interviewing Techniques and Styles. In I. Bourgeault, R. Dingwall \& R. deVries (Eds.), The SAGE Handbook of Qualitative Methods in Health Research (pp. 307-326). California: SAGE.

Kessler, R. C., Berglund, P. A., Coulouvrat, C., Hajak, G., Roth, T., Shahly, V., .. . Walsh, J. K. (2011). Insomnia and the performance of US workers: results from the America insomnia survey. Sleep, 34(9), 1161-1171.

Kleinman, L., Mannix, S., Yuan, Y., Kummer, S., L'Italien, G., \& Revicki, D. (2012). Review of patient-reported outcome measures in chronic hepatitis C. Health and Quality of Life Outcomes, 10, 92.

Kleinman, L., Zodet, M. W., Hakim, Z., Aledort, J., Barker, C., Chan, K., . . Revicki, D. (2000). Psychometric evaluation of the fatigue severity scale for use in chronic hepatitis C. Quality of Life Research, 9(5), 499-508. 
Knoop, H., Prins, J. B., Moss-Morris, R., \& Bleijenberg, G. (2010). The central role of cognitive processes in the perpetuation of chronic fatigue syndrome. Journal of Psychosomatic Research, 68(5), 489-494.

Knoop, H., van Kessel, K., \& Moss-Morris, R. (2012). Which cognitions and behaviours mediate the positive effect of cognitive behavioural therapy on fatigue in patients with multiple sclerosis? Psychological Medicine, 42(1), 205-213.

Kralik, D., Telford, K., Price, K., \& Koch, T. (2005). Women's experiences of fatigue in chronic illness. Journal of Advanced Nursing, 52(4), 372-380.

Krupp, L. B., LaRocca, N. G., Muir-Nash, J., \& Steinberg, A. D. (1989a). The fatigue severity scale. Application to patients with multiple sclerosis and systemic lupus erythematosus. Archives of Neurology, 46(10), 1121-1123.

Krupp, L. B., LaRocca, N. G., Muir-Nash, J., \& Steinberg, A. D. (1989b). The fatigue severity scale. Application to patients with multiple sclerosis and systemic lupus erythematosus. [Comparative Study

Research Support, U.S. Gov't, Non-P.H.S.]. Arch Neurol, 46(10), 1121-1123.

Kuo, G., Choo, Q. L., Alter, H. J., Gitnick, G. L., Redeker, A. G., Purcell, R. H., . . et al. (1989). An assay for circulating antibodies to a major etiologic virus of human non-A, non-B hepatitis. Science, 244(4902), 362-364.

Kyle, S. D., Morgan, K., Spiegelhalder, K., \& Espie, C. A. (2011). No pain, no gain: an exploratory within-subjects mixed-methods evaluation of the patient experience of sleep restriction therapy (SRT) for insomnia. Sleep Medicine, 12(8), 735-747.

Larun, L., \& Malterud, K. (2007). Identity and coping experiences in Chronic Fatigue Syndrome: a synthesis of qualitative studies. Patient Education and Counseling, 69(1-3), 20-28. 
Leavitt, V. M., \& DeLuca, J. (2010). Central fatigue: issues related to cognition, mood and behavior, and psychiatric diagnoses. PM R, 2(5), 332-337.

Lee, Hicks, G., \& Nino-Murcia, G. (1991). Validity and reliability of a scale to assess fatigue. Psychiatry Research, 36(3), 291-298.

Leeuw, M., Goossens, M. E., Linton, S. J., Crombez, G., Boersma, K., \& Vlaeyen, J. W. (2007). The fear-avoidance model of musculoskeletal pain: current state of scientific evidence. Journal of Behavioral Medicine, 30(1), 77-94.

Leger, D., Bayon, V., Laaban, J. P., \& Philip, P. (2012). Impact of sleep apnea on economics. Sleep Medicine Reviews, 16(5), 455-462.

Levethal, H., Nerenz, D.R., Steele D.J. (1984). Illness Representations and Coping with Health Threats. In A. Baum, Taylor, S.E., Singer, J.E (Ed.), Handbook of Psychology and Health, Volume IV: Social Psychological Aspects of Health. Hillsdale NJ: Erlbaum.

Lovibond, P. F., \& Lovibond, S. H. (1995). The structure of negative emotional states: comparison of the Depression Anxiety Stress Scales (DASS) with the Beck Depression and Anxiety Inventories. Behaviour Research and Therapy, 33(3), 335-343.

Lukkahatai, N., \& Saligan, L. N. (2013). Association of catastrophizing and fatigue: a systematic review. [Review]. J Psychosom Res, 74(2), 100-109. doi: 10.1016/j.jpsychores.2012.11.006

Luyster, F. S., Buysse, D. J., \& Strollo, P. J., Jr. (2010). Comorbid insomnia and obstructive sleep apnea: challenges for clinical practice and research. Journal of Clinical Sleep Medicine, 6(2), 196-204. 
Majer, M., Welberg, L. A., Capuron, L., Pagnoni, G., Raison, C. L., \& Miller, A. H. (2008). IFNalpha-induced motor slowing is associated with increased depression and fatigue in patients with chronic hepatitis C. Brain, Behavior, and Immunity, 22(6), 870-880.

Martinez-Martin, P., Catalan, M. J., Benito-Leon, J., Moreno, A. O., Zamarbide, I., Cubo, E., . . . Frades, B. (2006). Impact of fatigue in Parkinson's disease: the Fatigue Impact Scale for Daily Use (D-FIS). [s]. Quality of Life Research, 15(4), 597-606.

McCracken, L. M., \& Vowles, K. E. (2006). Acceptance of chronic pain. Current Pain and Headache Reports, 10(2), 90-94.

McCracken, L. M., \& Vowles, K. E. (2008). A prospective analysis of acceptance of pain and values-based action in patients with chronic pain. Health Psychology, 27(2), 215-220.

McCracken, L. M., Vowles, K. E., \& Eccleston, C. (2004). Acceptance of chronic pain: component analysis and a revised assessment method. Pain, 107(1-2), 159-166.

Mendoza, T. R., Wang, X. S., Cleeland, C. S., Morrissey, M., Johnson, B. A., Wendt, J. K., \& Huber, S. L. (1999). The rapid assessment of fatigue severity in cancer patients: use of the Brief Fatigue Inventory. Cancer, 85(5), 1186-1196.

Meyers, Elkashab, M., Ma, M., Crotty, P., \& Pomier-Layrargues, G. (2010). Transient elastography for the noninvasive assessment of liver fibrosis: A multicenter Canadian study. Canadian Journal of Gastroenterology, 24(11), 661-670.

Meyers, Ramji, A., Bilodeau, M., Wong, S., \& Feld, J. (2012). An update on the management of chronic hepatitis C: Consensus guidelines from the Canadian Association for the Study of the Liver. Canadian Journal of Gastroenterology, 26(6), 359-375. 
Michielsen, H. J., De Vries, J., \& Van Heck, G. L. (2003). Psychometric qualities of a brief selfrated fatigue measure: The Fatigue Assessment Scale. Journal of Psychosomatic Research, 54(4), 345-352.

Miles, J. N. V., \& Shevlin, M. (2001). Applying regression and correlation: a guide for students and researchers. London: Sage.

Montgomery, G. H., Kangas, M., David, D., Hallquist, M. N., Green, S., Bovbjerg, D. H., \& Schnur, J. B. (2009). Fatigue during breast cancer radiotherapy: an initial randomized study of cognitive-behavioral therapy plus hypnosis. Health Psychology, 28(3), 317-322.

Mor, N., \& Winquist, J. (2002). Self-focused attention and negative affect: a meta-analysis. Psychological Bulletin, 128(4), 638-662.

Morin, Belleville, G., Belanger, L., \& Ivers, H. (2011). The Insomnia Severity Index: psychometric indicators to detect insomnia cases and evaluate treatment response. Sleep, 34(5), 601-608.

Morin, LeBlanc, M., Daley, M., Gregoire, J. P., \& Merette, C. (2006). Epidemiology of insomnia: prevalence, self-help treatments, consultations, and determinants of helpseeking behaviors. Sleep Medicine, 7(2), 123-130.

Morin, Stone, J., Trinkle, D., Mercer, J., \& Remsberg, S. (1993). Dysfunctional beliefs and attitudes about sleep among older adults with and without insomnia complaints. Psychology \& Aging, 8(3), 463-467.

Morin, C. M. (1993). Insomnia: Psychological assessment and management. New York, NY: Guilford Press.

Morriss, R. (1993). Insomnia in the chronic fatigue syndrome. BMJ, 307(6898), 264. 
Moss-Morris. (2005). Symptom perceptions, illness beliefs and coping in chronic fatigue syndrome. Journal of Mental Health 14(3), 223-235.

Moss-Morris, Spence, M. J., \& Hou, R. (2011). The pathway from glandular fever to chronic fatigue syndrome: can the cognitive behavioural model provide the map? Psychological Medicine, 41(5), 1099-1107.

Moss-Morris, Weinman, J., Petrie, K. J., Horne, R., Cameron, L. D., \& Buick, D. (2002). The Revised Illness Perception Questionnaire (IPQ-R). Psychology and Health, 17, 1-16.

Moss-Morris, R., McCrone, P., Yardley, L., van Kessel, K., Wills, G., \& Dennison, L. (2012). A pilot randomised controlled trial of an Internet-based cognitive behavioural therapy selfmanagement programme (MS Invigor8) for multiple sclerosis fatigue. Behavoural Research Therapy, 50(6), 415-421.

Murphy, S. L., Smith, D. M., \& Alexander, N. B. (2008). Measuring activity pacing in women with lower-extremity osteoarthritis: a pilot study. [Research Support, N.I.H., Extramural Research Support, Non-U.S. Gov't]. Am J Occup Ther, 62(3), 329-334.

Nogueria, J. B. C., Sena, L.C.S., Souza SiqueriaQuintans, J., Silva Almeida, J.R.G., Franca, A.V.C., \& Junior, L.J.Q. (2012). Side effects of the therapy with peginterferon and ribavirin in chronic hepatitis C: A small audit. Journal of Pharmacy Practice, 25(1), 8588.

Ohayon, M. M. (2002). Epidemiology of insomnia: what we know and what we still need to learn. Sleep Medicine Reviews, 6(2), 97-111.

Ohayon, M. M., Riemann, D., Morin, C., \& Reynolds, C. F., 3rd. (2012). Hierarchy of insomnia criteria based on daytime consequences. Sleep Medicine, 13(1), 52-57. 
Okun, M. L., Reynolds, C. F., 3rd, Buysse, D. J., Monk, T. H., Mazumdar, S., Begley, A., \& Hall, M. (2011). Sleep variability, health-related practices, and inflammatory markers in a community dwelling sample of older adults. Psychosomatic Medicine, 73(2), 142-150.

Pepper, C. M., Krupp, L. B., Friedberg, F., Doscher, C., \& Coyle, P. K. (1993). A comparison of neuropsychiatric characteristics in chronic fatigue syndrome, multiple sclerosis, and major depression. The Journal of Neuropsychiatry and Clinical Neurosciences, 5(2), 200205.

Piper, B. F., Rieger, P. T., Brophy, L., Haeuber, D., Hood, L. E., Lyver, A., \& Sharp, E. (1989). Recent advances in the management of biotherapy-related side effects: fatigue. Oncology Nursing Forum, 16(6 Suppl), 27-34.

Poppe, C., Petrovic, M., Vogelaers, D., \& Crombez, G. (2013). Cognitive behavior therapy in patients with chronic fatigue syndrome: the role of illness acceptance and neuroticism. Journal of Psychosomatic Research, 74(5), 367-372.

Pottie, K., Greenaway, C., Feightner, J., Welch, V., Swinkels, H., Rashid, M., . . Tugwell, P. (2011). Evidence-based clinical guidelines for immigrants and refugees. Canadian Medical Association Journal, 183(12), E824-925.

Poynard, T., Cacoub, P., Ratziu, V., Myers, R. P., Dezailles, M. H., Mercadier, A., . . . Moussalli, J. (2002). Fatigue in patients with chronic hepatitis C. Journal of Viral Hepatitis, 9(4), 295-303.

Poynard, T., Imbert-Bismut, F., Munteanu, M., Messous, D., Myers, R. P., Thabut, D., . . . Hainque, B. (2004). Overview of the diagnostic value of biochemical markers of liver fibrosis (FibroTest, HCV FibroSure) and necrosis (ActiTest) in patients with chronic hepatitis C. Comparative Hepatology, 3(1), 8. 
Poynard, T., Ngo, Y., Perazzo, H., Munteanu, M., Lebray, P., Moussalli, J., ... Ratziu, V. (2011). Prognostic value of liver fibrosis biomarkers: a meta-analysis. Gastroenterology Hepatology (N Y), 7(7), 445-454.

Price, D. D., Finniss, D. G., \& Benedetti, F. (2008). A comprehensive review of the placebo effect: recent advances and current thought. Annual Review of Psychology, 59, 565-590.

Raison, C. L., Rye, D. B., Woolwine, B. J., Vogt, G. J., Bautista, B. M., Spivey, J. R., \& Miller, A. H. (2010). Chronic interferon-alpha administration disrupts sleep continuity and depth in patients with hepatitis C: association with fatigue, motor slowing, and increased evening cortisol. Biological Psychiatry, 68(10), 942-949.

Reichard, O., Norkrans, G., Fryden, A., Braconier, J. H., Sonnerborg, A., \& Weiland, O. (1998). Randomised, double-blind, placebo-controlled trial of interferon alpha-2b with and without ribavirin for chronic hepatitis C. The Swedish Study Group. Lancet, 351(9096), 83-87.

Repping-Wuts, H., Uitterhoeve, R., van Riel, P., \& van Achterberg, T. (2008). Fatigue as experienced by patients with rheumatoid arthritis (RA): a qualitative study. International Journal of Nursing Studies, 45(7), 995-1002.

Rhoten, D. (1982). Fatigue and the Postsurgical Patient. In C. Norris (Ed.), Concept clarification in nursing. (pp. 277-300). Rockville: Aspen Systems.

Rodin, G., Craven, J., \& Littlefield, C. (1991). Depression in the Medically Ill. New York, NY: Brunnel/Mazel Inc.

Sanchez-Ortuno, M. M., Edinger, J. D., \& Wyatt, J. K. (2011). Daytime symptom patterns in insomnia sufferers: is there evidence for subtyping insomnia? Journal of Sleep Research, 20(3), 425-433. 
Savard, M. H., Savard, J., Simard, S., \& Ivers, H. (2005). Empirical validation of the Insomnia Severity Index in cancer patients. Psychooncology, 14(6), 429-441.

Schillings, M. L., Kalkman, J. S., Janssen, H. M., van Engelen, B. G., Bleijenberg, G., \& Zwarts, M. J. (2007). Experienced and physiological fatigue in neuromuscular disorders. Clinical Neurophysiology, 118(2), 292-300.

Schwartz, J. E., Jandorf, L., \& Krupp, L. B. (1993). The measurement of fatigue: a new instrument. Journal of Psychosomatic Research, 37(7), 753-762.

Servaes, P., Gielissen, M. F., Verhagen, S., \& Bleijenberg, G. (2007). The course of severe fatigue in disease-free breast cancer patients: a longitudinal study. Psychooncology, 16(9), 787-795.

Servaes, P., Verhagen, S., \& Bleijenberg, G. (2002). Determinants of chronic fatigue in diseasefree breast cancer patients: a cross-sectional study. Annuals of Oncology, 13(4), 589-598.

Shahid, A., Wilkinson, K., Marcu, S., \& Shapiro, C. M. (2011). STOP, THAT and One Hundred Other Sleep Scales New York, NY: Springer.

Sharpe, M. (1997). Cognitive behavior therapy for functional somatic complaints. The example of chronic fatigue syndrome. Psychosomatics, 38(4), 356-362.

Shen, J., Barbera, J., \& Shapiro, C. M. (2006). Distinguishing sleepiness and fatigue: focus on definition and measurement. Sleep Medicine Reviews, 10(1), 63-76.

Sherman, M., Shafran, S., Burak, K., Doucette, K., Wong, W., Girgrah, N., ... Deschenes, M. (2007). Management of chronic hepatitis C: consensus guidelines. Canadian Journal of Gastroenterology, 21 Suppl C, 25C-34C.

Siegel, K., Lekas, H. M., \& Maheshwari, D. (2012). Causal attributions for fatigue by older adults with advanced cancer. Journal of Pain and Symptom Management, 44(1), 52-63. 
Sivertsen, B. (2013). The Illness Perception Questionnaire Retrieved August 8, 2012, from www.uib.no.ipq

Skerrett, T. N., \& Moss-Morris, R. (2006). Fatigue and social impairment in multiple sclerosis: the role of patients' cognitive and behavioral responses to their symptoms. Journal of Psychosomatic Research, 61(5), 587-593.

Smets, E. M., Garssen, B., Bonke, B., \& De Haes, J. C. (1995). The Multidimensional Fatigue Inventory (MFI) psychometric qualities of an instrument to assess fatigue. Journal of Psychosomatic Research, 39(3), 315-325.

Sockalingam, S., Blank, D., Al Jarad, A., Alosaimi, F., Hirschfield, G., \& Abbey, S. E. (2011). A comparison of depression screening instruments in hepatitis $\mathrm{C}$ and the impact of depression on somatic symptoms. Psychosomatics, 52(5), 433-440.

Spichiger, E., Rieder, E., Muller-Frohlich, C., \& Kesselring, A. (2012). Fatigue in patients undergoing chemotherapy, their self-care and the role of health professionals: a qualitative study. European Journal of Oncology Nursing, 16(2), 165-171.

Spielberger, C. D., Gorsuch, R. L., Lushhene, R., Vagg, P. R., \& Jacobs, G. A. (1983). Manual for the State-Trait Anxiety Inventory. Palo Alto, CA: Mind Garden.

Taylor, R. R., Jason, L. A., \& Torres, A. (2000). Fatigue rating scales: an empirical comparison. Psychological Medicine, 30(4), 849-856.

Teran-Santos, J., Jimenez-Gomez, A., \& Cordero-Guevara, J. (1999). The association between sleep apnea and the risk of traffic accidents. Cooperative Group Burgos-Santander. The New England Journal of Medicine, 340(11), 847-851.

Tsai, S. C., \& Lee-Chiong, T. (2013). Sleep Disorders and Fatigue. In M. Hirschkowitz \& A. Sharafkhaneh (Eds.), Fatigue (Vol. 8, pp. 235-240). New York, NY: Elsevier. 
Valko, P. O., Bassetti, C. L., Bloch, K. E., Held, U., \& Baumann, C. R. (2008). Validation of the fatigue severity scale in a Swiss cohort. Sleep, 31(11), 1601-1607.

Van Damme, S., Grombez, G., Van Houdenhove, B., Mariman, A., \& Michielsen, W. (2006). Well-being in patients with chronic fatigue syndrome:The role of acceptance. Journal of Psychosomatic Research, 61, 595-599.

van Kessel, K., \& Moss-Morris, R. (2006). Understanding multiple sclerosis fatigue: a synthesis of biological and psychological factors. J Psychosom Res, 61(5), 583-585.

van Kessel, K., Moss-Morris, R., Willoughby, E., Chalder, T., Johnson, M. H., \& Robinson, E. (2008). A randomized controlled trial of cognitive behavior therapy for multiple sclerosis fatigue. Psychosomatic Medicine, 70(2), 205-213.

van Weert, E., May, A. M., Korstjens, I., Post, W. J., van der Schans, C. P., van den Borne, B., . . . Hoekstra-Weebers, J. E. (2010). Cancer-related fatigue and rehabilitation: a randomized controlled multicenter trial comparing physical training combined with cognitivebehavioral therapy with physical training only and with no intervention. Physical Therapy, 90(10), 1413-1425.

Vgontzas, A. N., Papanicolaou, D. A., Bixler, E. O., Hopper, K., Lotsikas, A., Lin, H. M., .. . Chrousos, G. P. (2000). Sleep apnea and daytime sleepiness and fatigue: relation to visceral obesity, insulin resistance, and hypercytokinemia. The Journal of Clinical Endocrinology and Metabolism, 85(3), 1151-1158.

Vollestad, N. K. (1997). Measurement of human muscle fatigue. Journal of Neuroscience Methods, 74(2), 219-227. 
Vozoris, N. T. (2012). Sleep apnea-plus: prevalence, risk factors, and association with cardiovascular diseases using United States population-level data. Sleep Medicine, 13(6), 637-644.

Watson, N. F., Kapur, V., Arguelles, L. M., Goldberg, J., Schmidt, D. F., Armitage, R., \& Buchwald, D. (2003). Comparison of subjective and objective measures of insomnia in monozygotic twins discordant for chronic fatigue syndrome. Sleep, 26(3), 324-328.

Wessely, S., Chalder, T., Hirsch, S., Wallace, P., \& Wright, D. (1997). The prevalence and morbidity of chronic fatigue and chronic fatigue syndrome: a prospective primary care study. American Journal of Public Health, 87(9), 1449-1455.

Wessely, S., David, A. S., Butler, S., \& Chalder, T. (1989). Management of chronic (post-viral) fatigue syndrome. Journal of the Royal College of General Practitioners, 39, 26-29.

Wessely, S., \& Pariante, C. (2002). Fatigue, depression and chronic hepatitis C infection. Psychological Medicine, 32(1), 1-10.

White, P. D., Goldsmith, K. A., Johnson, A. L., Potts, L., Walwyn, R., DeCesare, J. C., . . . Sharpe, M. (2011). Comparison of adaptive pacing therapy, cognitive behaviour therapy, graded exercise therapy, and specialist medical care for chronic fatigue syndrome (PACE): a randomised trial. Lancet, 377(9768), 823-836.

WHO. (2011). World Health Organization Hepatitis C Fact Sheet. Retrieved from http://www.who.int/mediacentre/factsheets/fs164/en/ website:

Wiegand, J., \& Berg, T. (2013). The etiology, diagnosis and prevention of liver cirrhosis: part 1 of a series on liver cirrhosis. Deutsches Arzteblatt international, 110(6), 85-91. 
Yellen, S. B., Cella, D. F., Webster, K., Blendowski, C., \& Kaplan, E. (1997). Measuring fatigue and other anemia-related symptoms with the Functional Assessment of Cancer Therapy (FACT) measurement system. Journal of Pain and Symptom Management, 13(2), 63-74.

Yorkston, K. M., Johnson, K., Boesflug, E., Skala, J., \& Amtmann, D. (2010). Communicating about the experience of pain and fatigue in disability. Quality of Life Research, 19(2), 243-251.

Young, T., Skatrud, J., \& Peppard, P. E. (2004). Risk factors for obstructive sleep apnea in adults. JAMA, 291(16), 2013-2016.

Zeuzem, S., Feinman, S. V., Rasenack, J., Heathcote, E. J., Lai, M. Y., Gane, E., . . Brunda, M. J. (2000). Peginterferon alfa-2a in patients with chronic hepatitis C. The New England Journal of Medicine, 343(23), 1666-1672. 


\section{Abbreviations}

AHI apnea hypopnea index

ALT alanine aminotransferase

BMI body mass index

CHF chronic heart failure

CES-D Center for Epidemiological Studies Depression Scale

COPD chronic obstructive pulmonary disease

DASS Depression, Anxiety, Stress Scales

EEG electroencephalography

ELISA enzyme-linked immune sorbent essay

EMG electromyography

ESS Epworth Sleepiness Scale

fMRI functional magnetic resonance imaging

FSS Fatigue Severity Scale

HCV hepatitis C virus

HIV human immunodeficiency virus

IFN interferon

IL interleukin

INR international normalized ratio

PCA principle component analysis

PCR polymerase chain reaction

PSQI Pittsburg Sleep Quality Index

PET positron emission tomography 


$\begin{array}{ll}\text { pSS } & \text { primary Sjögren syndrome } \\ \text { RA } & \text { rheumatoid arthritis } \\ \text { REM } & \text { rapid eye movement sleep } \\ \text { SLE } & \text { systemic lupus erythematosus } \\ \text { Sleep SES } & \text { Sleep Self-efficacy Scale } \\ \text { THAT } & \text { Toronto Hospital Alertness Test }\end{array}$

